\title{
Role of protein kinase CK2 and phosphatase PP2A in Notch- mediated lateral inhibition
}

Anasua Bose

West Virginia University

Follow this and additional works at: https://researchrepository.wvu.edu/etd

\section{Recommended Citation}

Bose, Anasua, "Role of protein kinase CK2 and phosphatase PP2A in Notch-mediated lateral inhibition" (2010). Graduate Theses, Dissertations, and Problem Reports. 4565.

https://researchrepository.wvu.edu/etd/4565

This Dissertation is protected by copyright and/or related rights. It has been brought to you by the The Research Repository @ WVU with permission from the rights-holder(s). You are free to use this Dissertation in any way that is permitted by the copyright and related rights legislation that applies to your use. For other uses you must obtain permission from the rights-holder(s) directly, unless additional rights are indicated by a Creative Commons license in the record and/ or on the work itself. This Dissertation has been accepted for inclusion in WVU Graduate Theses, Dissertations, and Problem Reports collection by an authorized administrator of The Research Repository @ WVU.

For more information, please contact researchrepository@mail.wvu.edu. 


\title{
Role of protein kinase CK2 and phosphatase PP2A in \\ Notch-mediated lateral inhibition.
}

\section{Anasua Bose}

\author{
Dissertation submitted to the \\ Eberly College of Arts and Sciences \\ at West Virginia University \\ in partial fulfillment of the requirements \\ for the degree of
}

Doctor of Philosophy

In

Biological Sciences

Dr. Ashok Bidwai, PhD., Chair

Dr Clifton Bishop, Ph.D.

Dr. Kristine Krajnak, Ph.D.

Dr. Peter Mathers, Ph.D.

Dr. Karen Weiler, Ph.D.

Department of Biology

Morgantown, West Virginia

2010

Keywords: Drosophila melanogaster, CK2, PP2A, E(spl)M8, Notch 


\section{Abstract \\ Role of protein kinase CK2 and phosphatase PP2A in Notch mediated lateral inhibition}

\section{Anasua Bose}

The Notch signaling pathway is highly conserved in vertebrates and invertebrates and regulates binary cell fate specifications in diverse developmental contexts. In a process termed lateral inhibition, Notch signaling restricts a particular cell fate to a single cell from a group of multi-potential cells. This mechanism ensures precision in cell fate assignations, which are vital for histogenesis and organogenesis. Studies during Drosophila neurogenesis, specifically development of the compound eye and the mechanosensory bristles, have provided the most detailed insights into the underlying mechanisms. During this process, Notch signaling results in the selection of single R8 photoreceptors or bristle sensory organ precursors (SOP's) from proneural clusters that express the transcriptional activators encoded by atonal (ato) or genes of the achaete scute Complex (ASC), respectively. This selection is mediated through the $\mathrm{E}(\mathrm{spl})$ repressors, a group of evolutionarily conserved bHLH proteins that antagonize Ato or ASC. The emerging view is that repression by $\mathrm{E}(\mathrm{spl})$ proteins is a regulated process, one involving phosphorylation. The work described in this dissertation is aimed at characterizing the role of protein kinase CK2 and the phosphatase PP2A in lateral inhibition. The work of Chapter 2 provides in vivo evidence that loss of CK2 compromises lateral inhibition and results in the specification of supernumerary R8's and SOP's from proneural clusters. These effects appear to be sensitive to $E(s p l)$ dosage, consistent with the direct interaction of CK2 with a subset of $\mathrm{E}(\mathrm{spl})$ members. The eye and bristle phenotypes associated with loss of CK2 indicate that the activity of this kinase is essential for inhibitory Notch signaling. The work of Chapter 3 provides in vivo evidence that Widerborst (Wdb), the regulatory subunit of the phosphatase PP2A, is a participatory component of Notch signaling. Using gain- and loss-of-function studies in wild type flies, or those mutant for Notch and $E(s p l)$, it is demonstrated that Wdb is essential for PP2A activity, and that PP2A plays a role that is opposite to that of CK2. These opposing activities of CK2 and PP2A may involve direct 
interactions, and indicate that a regulatory nexus influencing $E(\mathrm{spl})$ activity lies at the heart of Notch signaling. The studies of Chapter 4 characterize a novel hypomorphic allele of CK2 to directly demonstrate a role for this kinase in eye and bristle development. In addition, the lethality of this hypomorphic allele has been rescued by the expression of a variant of the catalytic $(\alpha)$ subunit that exhibits temperature-sensitive (ts) behavior in vivo. This is the first ts-allele of CK2. Given the roles of CK2 in diverse developmental programs, compounded by the early lethality of null alleles of this kinase, flies rescued by ts-alleles of CK2 should represent a resource of wide utility to the Drosophila community. 


\section{Acknowledgement}

I would like to convey my sincere gratitude to the people who have been very supportive during the course of my doctoral pursuit. First and foremost I would like to thank my advisor Dr Ashok Bidwai for providing enormous support and guidance during this study. He has encouraged me to think critically and independently. I hope to carry forward the training I received from him in my future endeavors. I would also like to thank his family for hosting wonderful lab gatherings that have been a great source of enjoyment.

I greatly appreciate the valuable suggestions and guidance from my committee members, Dr. Clif Bishop, Dr. Kristine Krajnak, Dr. Peter Mathers and Dr Karen Weiler and the valuable insights from Dr Phil Keeting during the course of my study. I would like to thank all the members of my laboratory, Umesh, Pallavi, Bhaskar, Sophia, Jee-Un, Ezgi, Debolina, Chong, Mohna, Adam and Lucas whose help, support and friendship has been extremely valuable throughout my study. I greatly appreciate the help I received from my friends and colleagues at the Department of Biology. I would like to thank Diane Berry at NIOSH, Morgantown for SEM imaging. I am grateful to the Department of Biology for all the facilities and infrastructure to carry out research. I would like to thank all my friends in Morgantown for being like a family and for the enjoyable times that I shared with them.

Finally I would like to thank my parents, my parents-in-law, my husband, my brother, my sister-in-law and my little neice for their immense encouragement, love, support and sacrifices during this time. I would also like to convey my heartfelt gratitude to my extended family in India for their encouragement and support throughout the course of my study. 


\section{Table of Contents}

\begin{tabular}{|c|c|}
\hline Abstract & ii-iii \\
\hline Acknowledgement & iv \\
\hline Table of contents & $\mathrm{v}$ \\
\hline List of figures & vi-vii \\
\hline $\begin{array}{l}\text { Chapter } 1 . \\
\text { Introduction }\end{array}$ & $1-43$ \\
\hline $\begin{array}{l}\text { Chapter } 2 . \\
\text { Drosophila CK2 regulates lateral-inhibition during eye and bristle } \\
\text { development. }\end{array}$ & $44-77$ \\
\hline $\begin{array}{l}\text { Chapter } 3 . \\
\text { Role for PP2A in Notch-mediated lateral inhibition. }\end{array}$ & $78-108$ \\
\hline $\begin{array}{l}\text { Chapter } 4 \\
\text { Rescue of Drosophila CK2 } \alpha \text { mutants with temperature-sensitive alleles: A } \\
\text { resource for analysis of CK2 functions in development. }\end{array}$ & $109-131$ \\
\hline Future Perspectives & $132-134$ \\
\hline Bibliography & $135-149$ \\
\hline
\end{tabular}




\section{List of Figures}

\begin{tabular}{|c|c|}
\hline Figures & Page \\
\hline \multicolumn{2}{|l|}{ Chapter 1} \\
\hline Fig. 1. The different modes of Notch function in animal development. & 35 \\
\hline Fig. 2. Notch signaling pathway- Drosophila Notch receptor and the core components. & 36 \\
\hline $\begin{array}{l}\text { Fig. 3. Notch signaling pathway- a schematic representation of the regulatory } \\
\text { mechanisms }\end{array}$ & 37 \\
\hline $\begin{array}{l}\text { Fig. 4. Representation of Notch-mediated lateral inhibition during Drosophila R8 } \\
\text { specification. }\end{array}$ & 38 \\
\hline Fig. 5. R8 specification and retinal patterning in the Drosophila compound eye. & 39 \\
\hline $\begin{array}{l}\text { Fig. 6. Patterning and morphogenesis of the Drosophila bristle mechanosensory } \\
\text { organs. }\end{array}$ & 40 \\
\hline Fig. 7. Domain organization and regulation of E(spl) M8. & 41 \\
\hline $\begin{array}{l}\text { Fig. 8. Structure of the CK2 holoenzyme and the alleles of Drosophila CK2 } \alpha \text { subunit } \\
\text { are represented. }\end{array}$ & 42 \\
\hline $\begin{array}{l}\text { Fig. 9. The structure of PP2A holoenzyme and its regulatory subunits in Drosophila are } \\
\text { represented. }\end{array}$ & 43 \\
\hline \multicolumn{2}{|l|}{ Chapter 2} \\
\hline $\begin{array}{l}\text { Fig. 1. Summary of eye phenotypes elicited by (mis)expression of } \mathrm{E}(\mathrm{spl}) \mathrm{m} 8 \text { and its } \\
\text { CK2 specific variants. }\end{array}$ & $68-69$ \\
\hline Fig. 2. Compromising CK2 elicits eye defects. & $70-71$ \\
\hline Fig. 3. The rough eye due to CK2-RNAi correlates to the onset of retinal patterning. & 72 \\
\hline Fig. 4. Compromising CK2 elicits supernumerary R8's. & 73 \\
\hline Fig. 5. Compromising CK2 elicits bristle patterning defects. & 74 \\
\hline Fig. 6. Mutations in $C K 2$ or $E(s p l)$ modulate bristle defects of CK2 compromised flies. & $75-76$ \\
\hline Fig. 7. Roles for CK2 during lateral inhibition. & 77 \\
\hline \multicolumn{2}{|l|}{ Chapter 3} \\
\hline Fig.1. CK2-RNAi suppresses the bristle defects of ectopic M8. & 101 \\
\hline $\begin{array}{l}\text { Fig. 2. Increased dosage of wdb elicits bristle and wing defects akin to Notch loss of } \\
\text { function. }\end{array}$ & 102 \\
\hline Fig. 3. Wdb modulates the retinal defects in $N^{s p l} / Y$. & 103 \\
\hline Fig. 4. Wdb effects on $N^{\text {spl }}$ reflect R8 specification. & 104 \\
\hline $\begin{array}{l}\text { Fig. 5. Increased wdb dosage rescues the rough and reduced eye of } N s p l /+; E(s p l) D /+ \\
\text { flies. }\end{array}$ & $105-106$ \\
\hline Fig. 6. Increased wdb dosage rescues the dominant retinal defects of ectopic M8SD. & $107-108$ \\
\hline Cha & \\
\hline
\end{tabular}




\begin{tabular}{|l|l|}
\hline Fig. 1. Molecular modeling of the TS variants of Drosophila CK2 $\alpha$. & 123 \\
\hline $\begin{array}{l}\text { Fig. 2. A schematic representation of of the Drosophila CK2 } \alpha \text { mutants and their } \\
\text { developmental progression. }\end{array}$ & 124 \\
\hline Fig. 3. Phenotypic analysis of homozygous CK2 & $125-126$ \\
\hline Fig. 4. Compromised CK2 activity elicits morphogenetic furrow defects. & 127 \\
\hline $\begin{array}{l}\text { Fig. 5. A schematic representation of the constructs employed for rescue of } \\
\text { homozygous null mutants of Drosophila CK2 } \alpha .\end{array}$ & 128 \\
\hline $\begin{array}{l}\text { Fig. 6. The scheme for generation and verification of rescue stock. } \\
\text { Fig. 7. Phenotypic analysis of rescue flies at restrictive temperature. }\end{array}$ & 129 \\
\hline
\end{tabular}


Chapter 1

Introduction 
Almost 40 years ago Lewis Wolpert coined the term 'pattern formation' to describe the signaling mechanisms that regulate development (Wolpert, 1978). Pattern formation encompasses cellular and molecular mechanisms such as asymmetric cell division, cell-cell communication, cell migration, growth and apoptosis. These processes regulate cell specification, cell differentiation and tissue morphogenesis in a spatial and temporal manner. They may occur either sequentially or simultaneously, giving rise to precise patterns in tissues, organs and in whole animals. Pattern formation begins with the establishment of the antero-posterior (AP) and the dorso-ventral (DV) axes in the embryo, and this is subsequently followed by the formation of the germ layers, i.e., the ectoderm, the mesoderm and the endoderm. Following this, the three dimensional form of the organism arises through extensive cell migration events such as gastrulation, where cells from the surface of the embryo move inwards giving rise to the gut. Later, the processes of cell specification and cell differentiation are initiated, whereby different cell types are specified leading to the formation of distinct tissues and organs. The above four processes are finally followed by growth and apoptosis that determine the final form of the organism.

These patterning events require intricate communication between cells. Through a panoply of biochemical, cellular, molecular and genetic studies, it has emerged that cell-cell communications require complex networks of interacting proteins, which constitute signal transduction pathways. Although metazoan organisms display an array of body plans, the underlying signaling pathways that orchestrate development are highly conserved across large phylogenetic distances. The five major and highly conserved signaling pathways that have been well characterized during development are Decapentaplagic (Dpp), Hedgehog $(\mathrm{Hh})$, Wingless (Wg), Epidermal Growth Factor Receptor (EGFR) and Notch (N). Of these, the Notch signaling pathway is distinct in that it involves communication only between adjacent cells. As a consequence, this pathway signals in a juxtracrine manner, unlike the other four (Dpp, $\mathrm{Hh}, \mathrm{Wg}$, and EGFR), which are endocrine or paracrine in nature. Notch is 
involved in binary cell fate specification during which the cell that receives this signal acquires a fate distinct from that of the cell that sends the signal. This binary cell fate specification is reiteratively employed during animal development for proper patterning of tissues. Therefore, the distinct steps of the Notch signaling pathway must be precisely regulated. This introduction is focused on the Notch signaling pathway, emphasizing its organization, architecture, regulation and roles in cell fate specification.

\section{Notch signaling pathway and animal development:}

About 100 years ago Notch was identified in the laboratory of Thomas Hunt Morgan through studies of mutations in Drosophila that displayed 'notched wings'. The notched wing phenotype was attributed to loss of wing tissue, although at that time the identity of the Notch gene and its roles in animal development were largely unknown. Twenty years later, David Poulson described embryonic lethal mutations that exhibited neural hypertrophy and at the same time lacked epidermal tissue, a phenotype that has been termed 'neurogenic' (Poulson, 1937). These neurogenic phenotypes in embryos resulted from the specification of an excess of neuronal cells at the expense of epidermal cells. These observations laid the foundation for later studies, which identified and characterized the role of Notch signaling in animal development. Over the years, additional genetic loci were identified that also exhibited neurogenic phenotypes. Epistasis analysis of these neurogenic loci eventually resulted in the description of the components of the Notch signaling pathway, their order, and their roles during diverse developmental paradigms (Knust et al., 1987; Lehmann et al., 1983;

Schweisguth and Posakony, 1992; Shepard et al., 1989; Simpson, 1990). It was studies in the Drosophila model that, in fact, set the stage for subsequent analysis of this pathway in the mouse model system and the correlation of the defects in this pathway to the etiology of several human developmental defects (reviewed in Bier, 2005; Cauchi and van den Heuvel, 2006). 
At present, Notch signaling is known to play roles in numerous developmental contexts ranging from neurogenesis, somitogenesis, oogenesis, left-right asymmetry, vascular development, kidney development and the regulation of stem cells (reviewed in ArtavanisTsakonas et al., 1999; Lai, 2004; Mumm and Kopan, 2000). Notch signaling is involved in both the restriction and the induction of cell fates. During development, Notch functions can be primarily classified into three modes (reviewed in Artavanis-Tsakonas et al., 1999; Bray, 2006; Lai, 2004). These include lateral inhibition, lineage decisions and boundary formation (Fig. 1). During lateral inhibition, Notch restricts cell fate specification to a single cell from a group of equipotential cells, thereby ensuring that only the proper number and the type of cells are specified and patterned within a given tissue. Lineage decisions occur during asymmetric cell division when one cell inherits a Notch regulator and the other does not. Consequently, the two resulting daughter cells display differential activities of Notch, which then drive the formation of distinct cell lineages. During boundary formation, a population of cells induces Notch activity in an adjacent population of cells that leads to their segregation into two distinct cell types. During development, however, Notch is employed not only for cell fate specification, but also for the regulation of cell proliferation, the maintenence of stem cells, as well as apoptosis (reviewed in Artavanis-Tsakonas et al., 1999; Miele and Osborne, 1999).

This introduction will mainly focus on the role of Notch in the execution of lateral inhibition during neurogenesis, emphasizing its functions in the formation of two sensory organs of Drosophila. These include, the compound eye, a component of the central nervous system, and the mechanosensory bristles, a component of the peripheral nervous system.

\section{The components of the Notch signaling pathway:}

The core components of this pathway in Drosophila are the transmembrane receptor Notch (N), its ligands Delta (DI) or Serrate (Ser), the transcription factor Suppressor of Hairless $(\mathrm{Su}(\mathrm{H}))$ and the basic helix loop helix $(\mathrm{bHLH})$ repressors encoded by the Enhancer of 
split Complex $(E(s p l) C)$ (Delidakis and Artavanis-Tsakonas, 1991; Hartley et al., 1988; Klambt et al., 1989; Knust et al., 1992; Schweisguth and Posakony, 1992).

The $E(s p l) C$ locus was first discovered by virtue of a unique genetic interaction with the split allele of the X-linked gene Notch. Heretofore, this allele is referred to as $N^{s p l}$. A unique dominant mutation in the $E(s p l)$ locus, called $E(s p l) D$, was identified more than 50 years ago based on its ability to 'enhance' the eye defect characteristic of the $N^{\text {spl }}$ mutation (Welshons, 1956). The name Enhancer of split reflects this genetic interaction. At that time, the identity, complexity of this locus or the underlying lesion(s) remained to be defined. Later studies revealed that the $E(s p l) C$, which is located on third chromosome, is inordinately dense. It encodes for a family of structurally similar basic-helix-loop-helix (bHLH) repressors called $M \beta$, $\mathrm{M} \gamma, \mathrm{M} \delta, \mathrm{M} 3, \mathrm{M} 5, \mathrm{M} 7$, and M8, their common and obligate co-repressor Groucho (gro), and a number of non-translated RNA's (Delidakis and Artavanis-Tsakonas, 1991; Hartley et al., 1988; Klambt et al., 1989; Knust et al., 1992; Lai et al., 2005b). With the sequencing of the genomes of several Drosophila species, it has emerged that not only are all of these transcription units conserved, but that the order of their appearance is invariant. This remarkable conservation of transcript order suggests that the cis-regulatory code(s) driving tissue specific expression of individual members is likely to be highly complex. It is, perhaps, for this very reason that the $E(s p l) D$ mutation is a fortuitous lesion in one transcription unit, that encoding for the M8 protein. The $\mathrm{E}(\mathrm{spl})$ repressors are the final transcriptional targets of activated Notch in lateral inhibition. The vertebrate counterparts of the Drosophila E(spl) repressors have been named the Hairy-enhancer of split like repressors, the HES proteins (Sasai et al., 1992; Stifani et al., 1992). As in Drosophila, the HES proteins in mammals are known to function in both inhibitory and inductive modes of Notch signaling (reviewed in Artavanis-Tsakonas et al., 1999; Lai, 2004).

\section{Structure of Drosophila Notch:}


Drosophila Notch is a $300-k d$ single pass transmembrane receptor. The extracellular domain (Fig. 2) is characterized by 29-36 Epidermal Growth Factor like repeats (EGF-repeats) and three highly conserved cysteine rich LIN-12/Notch repeats (LNR's), which are followed by a heterodimerization domain (HD) that is adjacent to the transmembrane region (reviewed in Tien et al., 2009). The HD domain harbors sites for proteolytic cleavage, which is an essential step in the activation of the Notch receptor (Gordon et al., 2007). The ligands Delta and Serrate have highly conserved N-terminal MNNL and Delta-Serrate ligand (DSL) domains, which are followed by EGF repeats and a Cysteine rich (CR) domain in close proximity to the transmembrane region (Fig. 2). The differences between the affinity/specificity of Notch for its ligands reflect the number of EGF repeats, and either the presence or the absence of the CR domain. The conserved MNNL and DSL domain are required for the binding of Delta and Serrate with Notch (reviewed in Gordon et al., 2008; Tien et al., 2009). In case of the Notch receptor, each EGF-repeat is comprised of approximately 40 amino acids and is held together with six cysteine residues linked by disulphide bonds (Fleming, 1998). A gain or loss of cysteine residues in these EGF-repeats results in the formation of aberrant disulphide bonds, and have been associated with Notch gain of function alleles, such as abruptex that display the loss of wing veins (de Celis and Garcia-Bellido, 1994; Fryxell et al., 2001). Similar defects in the Notch3 isoform are associated with the pathology of the human disease CADASIL (cerebral autosomal dominant arteriopathy with subcortical infarcts and leukoencephalopathy) (Joutel et al., 1996; Tournier-Lasserve et al., 1991).

To date, orthologs of the core components of Notch signaling pathway have been isolated across phyla from sea urchins to vertebrates including humans (Gridley, 1997; Sherwood and McClay, 1997). Unlike the single Notch gene in Drosophila, mammals contain four distinct isoforms of the Notch receptor. In mammals, multiple genes also encode for the ligands DI and Ser (Fig. 2). In addition to the above core components, a number of proteases, glycosyltransferases, ubiquitin ligases and transcriptional co-factors are required for proper 
Notch signaling, and these components are likewise also conserved.

\section{Regulation of Notch signaling:}

Given that Notch is required for cell fate specification in numerous developmental contexts the strength and timing of Notch activity is very critical. An immense body of evidence indicates that post- translational modifications regulating receptor and ligand maturation, endocytosis, protein trafficking and transcriptional switching are fundamental to the regulation of Notch signaling (Fig. 3). In addition, the molecular conformation of core components of Notch signaling has provided additional insights into regulation. The sections below provide a summary of these regulatory mechanisms.

\section{Receptor maturation:}

Notch undergoes successive post-translational modifications such as proteolytic cleavage and glycosylation to form the mature receptor (Fig. 3). Initially, Notch is synthesized as a monomer that undergoes proteolytic cleavage into the Notch extracellular and intracellular domains that are referred to as NECD and NICD, respectively. Heterodimerization of these two domains via non-covalent interactions then results in a membrane-anchored form of the receptor. The first cleavage, which is referred to as the 'S1 cleavage', occurs in the Golgi complex, and this reaction is catalyzed by the Furin-like convertases (Logeat et al., 1998).

In addition to this initial proteolytic cleavage, the Notch receptor also undergoes succesive glycosylation in the endoplasmic reticulum and Golgi complex before it is targeted to the plasma membrane as a mature receptor (Fig. 3). The EGF-like repeats of the extracellular domain of Notch are targets of modification by glycosylation, and these play important roles in receptor sensitization/desensitization (see below). These reactions are catalyzed by the glycosyltransferases. The role of glycosylation in Notch signaling was first identified through one such glycosyltransferase, Fringe (Moloney et al., 2000). During 
receptor maturation in the Golgi apparatus, Fringe catalyzes the transfer of $\mathrm{N}$ acetylglucosamine (GlcNAc) to the EGF-like repeats of the Notch receptor (Fig. 3). Both cell culture and in vivo studies indicate that Fringe can only transfer GlcNAc to O-fucosylated EGF-like repeats, indicating that fucosylation is a prior requirement (Bruckner et al., 2000). The initial fucosylation of the EGF-like repeats is catalyzed by O-fucosyltransferase, OFUT-1 in the endoplasmic reticulum (Fig. 3) (Okajima et al., 2003).

Studies in the developing Drosophila wing indicate that modification of the Notch extracellular domain by Fringe enhances interactions of this receptor with Delta, but does not appear to modulate the strength of binding of Serrate (Fleming et al., 1997; Klein and Arias, 1998; Panin et al., 1997). Fringe is required for the determination of the dorso-ventral boundary of the wing imaginal disc. Homologues of Fringe in vertebrates have been identified. As in Drosophila, these potentiate the Notch-Delta interaction, but inhibit the NotchJagged (Serrate homologue) interaction (Hicks et al., 2000). However, Fringe is required for only a subset of Notch functions that are mainly operational during inductive signaling, but appear to not be required during asymmetric cell division or lateral inhibition (Haines and Irvine, 2003). It should, however, be noted that OFUT-1 is required for all modes of Notch signaling including lateral inhibition, lineage specification and inductive signaling. Initial evidence for the requirement of O-fucosylation came from the analysis of mutations in the biosynthesis of GDP-fucose that affected Notch activity (Moloney et al., 2000). Consistent with the presence of consensus sites for fucosylation, the EGF repeats of the Notch receptor are targets for this modification. In addition, both in vitro and in vivo studies in Drosophila and mice indicate that loss of Ofut-1 function prevents the O-fucosylation of Notch resulting in a non-functional receptor (Okajima and Irvine, 2002; Sasamura et al., 2003).

Studies of glycosylation mutants have provided additional insights into regulation of Notch activity. The O-fucosylation site in the EGF repeat-12 is highly conserved in all Notch receptors, and the EGF repeats 11 and 12 are particularly critical for ligand interaction (Rebay 
et al., 1991). Both OFUT-1 and Fringe target the EGF repeat 12 (Shao et al., 2003). Several Notch mutants have been isolated with specific mutations in EGF repeats such as $N^{M 1}$, which harbors a missense mutation in the EGF repeat 12 leading to a strong Notch loss of function phenotype (de Celis et al., 1993). Point mutations in the EGF repeat 24, 25, 27 and 29 are associated with the Abruptex $\left(N^{A x}\right)$ class of mutations, which display characteristics of a gain of Notch function (de Celis and Bray, 2000; de Celis and Garcia-Bellido, 1994; Hartley et al., 1987; Kelley et al., 1987). Although mutations in fringe genetically interact with $N^{A x}$ alleles, the biochemical basis still remains to be defined.

Recent biochemical and structural analysis of Notch has provided insights into potential mechanism underlying the gain of function nature of the Abruptex mutations. These indicate that the EGF repeats 11-20 interact with repeats $21-30$, possibly in an intramolecular manner (Pei and Baker, 2008). Given that repeats 11 and 12 are vital for interaction with the ligand, the repeats 21-30 might impose 'autoinhibition' to prevent or compete with ligand binding. As stated above, the repeats $21-30$ are particularly relevant since they are associated with the Abruptex mutations. It has been suggested that mutations in these repeats may impair the intramolecular 'autoinhibition' of repeats 11-12. Because of this lack of inhibition the mutant Notch receptor displays enhanced interactions with ligands, thereby resulting in a gain of Notch activity (Pei and Baker, 2008). In a similar manner, glycosylation is also thought to alter the conformations of the EGF repeats, themselves, to influence the strength of their interaction with the ligands.

\section{The $\boldsymbol{N}^{s p l}$ mutation:}

The role of altered glycosylation in modulating Notch signaling is underscored by the split allele, $N^{s p l}$. In the $N^{s p l}$ allele, an ectopic O-fucosylation site is introduced due to the substitution of an Ile residue of EGF-repeat 14 with a Thr (Hartley et al., 1987; Kelley et al., 1987). Consequently, this replacement facilitates mis-glycosylation by OFut-1 and Fringe, 
resulting in a receptor that displays greater signaling strength. It has been reported that during Drosophila eye development, the mutant $N^{\text {spl }}$ receptor exhibits increased interaction with Delta, and consequently results in a gain of Notch activity. This has been well documented in the case of the precursors of the R8 photoreceptors (Li et al., 2003). It has, therefore, been suggested that the EGF repeat 14 might normally be involved in inhibiting Notch signaling. Given that $N^{s p l}$ also perturbs bristle patterning, the possibility remains open that this mutation enhances receptor activity beyond a threshold during bristle development, although this has not been formally demonstrated. It is important to note that even though Notch plays roles in other developmental contexts (see above), the $N^{\text {spl }}$ mutation does not elicit defects in wing morphogenesis, or during oogenesis or myogenesis. It is, therefore, possible that $N^{s p l}$ may predominantly affect neurogenesis. Together these findings indicate that glycosylation is most likely a determinant of EGF repeat conformations in the mature receptor and regulates Notch activity.

\section{Endosomal Sorting:}

The initial evidence for regulation of Notch receptor by endocytosis emerged from studies in temperature-sensitive (ts) mutants of Drosophila Dynamin, shibire $\left(s h i^{t s}\right)$. The shits flies exhibited paralysis at high temperature, but were normal at low temperatures (Poodry, 1990). Dynamin is a cytosolic GTPase that is required for endocytosis of vesicles from the plasma membrane. Dynamin polymerizes around the neck of a clathrin-coated pit during endocytosis, and subsequently, GTP hydrolysis leads to subunit contraction and facilitates endocytosis of the vesicle from the plasma membrane. Initial studies indicated that in shits mutants, Notch signaling is impaired in both the signal receiving and the signal sending cells (Poodry, 1990; Seugnet et al., 1997).

Numb is another conserved and membrane associated protein known to interact with Notch (Fig. 3) and promote endocytosis via clathrin-coated vesicles (Santolini et al., 2000). 
Clathrin is a fibrous protein that polymerizes to form a coat around membrane bound vesicles. Clathrin is stabilized by assembly particles (AP), that are adapter proteins required to promote polymerization of Clathrin, bind to the vesicular membrane proteins and determine particle inclusion in the vesicle. Direct interaction of Numb with both Notch and $\alpha$-adaptin, a subunit of Clathrin-AP2 adapter complex indicates that Numb may facilitate Notch endocytosis (Berdnik et al., 2002). The function of Numb in Notch signaling was determined through studies in binary cell fate decisions in Drosophila sensory organ precursor lineage. In this developmental context, Numb is asymmetrically localized and therefore becomes segregated into one of the two daughter cells. Notch signaling is inhibited in only the daughter cell that inherits Numb (Frise et al., 1996; Guo et al., 1996). Furthermore, Numb also inhibits membrane accumulation of a four-pass transmembrane protein known as Sanpodo that is required for Notch signaling in Drosophila (O'Connor-Giles and Skeath, 2003). It has also been found that in mammals Numb mediates ubiquitinylation of the Notch receptor by promoting its interaction with the E3 ubiquitin ligase, called Itch (McGill and McGlade, 2003). Both, in case of Sanpodo and Itch, Numb decreases the levels of the Notch receptor at the cell surface, indicating its roles as inhibitor of Notch signaling.

Ubiquitinylation and internalization of Notch leads to either degradation or recycling to the plasma membrane (Fig. 3). Several ubiquitin ligases are involved in directing Notch toward degradation or recycling. One such ubiquitin ligase in Drosophila, Deltex (Dx), increases the sorting to endosomal compartments, but this is thought to protect Notch from degradation (Hori et al., 2004). It has been suggested that a second E3 ubiquitin ligase, Suppressor of Deltex $(\mathrm{Su}(\mathrm{dx}))$, acts antagonistically to Dx to also regulate Notch endosomal sorting. In addition to ubiquitin ligases, several proteins involved in vesicular trafficking are regulators of Notch signaling. One example is the 'endosomal sorting complex required for transport', called ESCRT. ESCRT is essential for the recruitment, transfer and concentration of ubiquitinylated cargo in endosomes for degradation. Normally, after internalization Notch is 
either degraded in lysosomes via multivesicular bodies (MVBs) or recycled back to the plasma membrane (Fig.3). Mutations in the components of the ESCRT complex lead to aberrant accumulation of Notch in enlarged endosomes (Thompson et al., 2005; Vaccari and Bilder, 2005). It is predicted that $\gamma$-secretase associated with the endosomes might lead to proteolytic cleavage and release of Notch intracellular domain thereby increasing Notch activity, even in the absence of a relevant ligand (reviewed in Le Borgne, 2006). The above reported studies indicate that endosomal sorting is an essential mechanism for the regulation of the Notch receptor.

\section{Ligand maturation:}

In line with the regulation of the Notch receptor, ubiquitinylation and endocytic trafficking of ligands are also crucial for Notch signaling (Fig. 3) (Chitnis, 2006; Le Borgne et al., 2005). A Drosophila Epsin gene, Liquid facet (Lqf) has been found to be required for Notch signaling (Overstreet et al., 2004; Wang and Struhl, 2004). Epsins are a family of adapter proteins that interact with components of the Clathrin coat. During ligand maturation, Epsin mediated endocytosis is required to produce an active ligand (Overstreet et al., 2004; Wang and Struhl, 2004). In addition, ubiquitinylation of DSL ligands facilitates the interaction with Epsins that leads to the process of endocytosis. Ubiquitinylation of DSL ligands are catalyzed by two ring-finger E3 ubiquitin ligases, Neuralized and Mind bomb (Itoh et al., 2003; Lai et al., 2005a; Le Borgne et al., 2005). These ubiquitin ligases were identified through studies in cell lineage specification of Drosophila sensory organ precursors (SOPs). The SOP undergoes asymmetric cell divisions and ubiquitin ligases such as Neuralized become unequally distributed in the two daughter cells (Le Borgne and Schweisguth, 2003). In contrast to Numb, which is a negative regulator of Notch signaling, Neuralized increases

Delta-Serrate activity, thereby activating Notch signaling in the adjacent cell. Studies also indicate that Neuralized, Mindbomb and Epsin function in a non-cell autonomous manner. 
This suggests that endocytosis of DSL ligands is required for Notch signaling. In addition, Epsin mediated recycling of ligands is also dependent on small GTPase Rab11 and Sec15, a component of the exocyst. Sec15 recognizes vesicles bound by GTPases, and regulates vesicular trafficking. Mutations in Rab11 and Sec15 have been implicated in the transformation of cell lineages during Drosophila SOP specification (Emery et al., 2005; JafarNejad et al., 2005). In addition to ligand maturation, endocytosis of receptor bound ligand is crucial for proteolytic cleavage of Notch extracellular domain, an important step for activation of Notch signaling.

\section{Regulated proteolysis}

In addition to glycosylation and endocytosis, proteolytic cleavage adds another layer of regulation to Notch signaling. Notch signaling is activated upon successive proteolytic cleavages. Subsequent to the initial (S1) cleavage of the Notch precursor (see above), the ADAM family of proteases mediates the $S 2$ cleavage, which is necessary for Notch signaling. In the unoccupied receptor, the adjacent LNR domain sterically hinders the HD domain to occlude the S2 cleavage site. Upon ligand binding, however, the protected S2 cleavage site now becomes exposed. This conformational state of the receptor can then be modified by the ADAM proteases (Brou et al., 2000; Gordon et al., 2007). It is thought that in addition to ligand binding, endocytosis of the Notch extracellular domain also provides a mechanical force that 'pulls' the LNR domain away from the HD domain. This physical disruption of interdomain contacts has been proposed to underlie Notch activation in a target cell. Such a mechanism would prevent ectopic activation of Notch in the absence of a ligand, and more importantly would only occur in the context of two adjacent cells, i.e., cell-cell contact (reviewed in Gordon et al., 2008). This model also takes into account the observations that a soluble form of the ligand Delta is insufficient to activate Notch processing in cell cultures, or to the transcriptional activation of target genes such as $E(s p l)$ (Hicks et al., 2002; Sun and 
Artavanis-Tsakonas, 1997). Thus juxtacrine signaling by Notch is intricately linked to its presentation, processing, and endocytosis. Following S2 cleavage, the Notch extracellular truncation domain (NEXT) undergoes proteolytic cleavage (S3 cleavage) by $\gamma$-secretase that releases the NICD (Fig. 3, (Okochi et al., 2002)). Cleavage by $\gamma$-secretase can also occur in the endosomal compartment following ubiquitinylation and internalization of NEXT, and there is debate in the field whether the plasma membrane or endosomal component of Notch is the site for activation by multiple proteases (Gupta-Rossi et al., 2004). Thus regulated proteolytic cleavage and endocytosis of Notch are intimately connected and are crucial regulators of Notch signaling.

\section{Transcriptional switch and target gene expression:}

Activation of $\mathrm{N}$ in the signal-receiving cell is followed by proteolytic cleavage and the release of NICD, which lead to a cascade of intracellular events. Specifically NICD translocates to the nucleus and associates with $\mathrm{Su}(\mathrm{H})$ or CSL (CBF-Su(H)-Lag-1) proteins. This interaction switches these repressors into transcriptional activators (Fig. 3), thereby providing a 'binary' type encoding for the target genes. CSL proteins drive transcription of the terminal Notch effectors, the bHLH E(spl)/HES repressors.

The association between NICD and CSL occurs through specific binding domains that recruit co-activators such as Mastermind (Mam) in Drosophila or Mam-like (MAML) in mammals (Petcherski and Kimble, 2000). Mam recruits HAT's and p300 leading to the assembly of the transcription initiation and activation complexes (Fryer et al., 2002). The functional regions in NICD are RAM (recombination binding protein-J associated molecule) domain, a nuclear localization signal (NLS), six ankyrin (Ank) repeats, transcriptional activation (TAD) domain and a C-terminal PEST motif (Kurooka et al., 1998; Tamura et al., 1995). The RAM region is required for the binding of NICD with CSL. The basic structure of CSL is comprised of three domains an N-terminal and C-terminal Rel-homology region (RHR- 
N and RHR-C) and a central $\beta$-trefoil domain (BTD) (Kovall and Hendrickson, 2004).

Recent structural analysis of human and worm NICD-CSL-MAM complex bound to DNA has provided novel insights into the conformation and domain functions of NICD (Nam et al., 2006; Wilson and Kovall, 2006). The seven ANK repeats in NICD are highly conserved and each is composed of 33 residues that form a pair of anti-parallel helices. It appears to be the case that the concave part of the ANK domain fits between the RHR-domains of CSL, thereby providing the interface for binding MAM. On the other hand, the $\mathrm{N}$-terminal region of the RAM domain binds to a groove in the BTD domain through a WxP motif. It was observed that C-terminal part of the RAM domain folds onto the N-terminus of the ANK domain and stabilizes the first ANK repeat. Moreover, the RAM domain has a higher affinity for CSL than the ANK domain. Based on these studies it has been suggested that the RAM domain interacts with CSL prior to ANK domain recruitment, and then stabilizes the ANK domain and CSL. This is followed by MAM recruitment (Gordon et al., 2008). The NICD-CSL-MAM transcriptional switch is downregulated via phosphorylation and degradation of NICD. The PEST domain in NICD is targeted by kinases such as CDK8 and subsequently undergoes proteosomal degradation (Tsunematsu et al., 2004). This leads to a termination of Notch signaling and CSL then complexes with co-repressors to actively turn off the transcription of target genes.

\section{Notch signaling and lateral inhibition:}

During neurogenesis, bHLH transcription factors derived from the atonal (ato) or the achaete-scute Complex (ASC) are expressed in groups of cells, the proneural clusters (PNC's, (Heitzler et al., 1996; Jarman et al., 1995; Jarman et al., 1994)). The activities of these transcription factors (proneural activators) bestows neural competency on all the cells of the PNC (Calleja et al., 2002; Dambly-Chaudiere and Vervoort, 1998; Gibert and Simpson, 2003). However, only a specified number of cells from each PNC adopt the neural cell fate, 
and these cells become sensory organ precursors (SOPs). This restriction is known as lateral inhibition (Lehmann et al., 1983; Simpson, 1990). Specifically, the future SOP expresses the ligand Delta at a level sufficient to induce Notch activity in the neighboring cells of the PNC, i.e., the non-SOPs. This triggers the release of the Notch intracellular domain $\left(\mathrm{N}^{\text {icd }}\right)$, which translocates to the nucleus and associates with $\mathrm{Su}(\mathrm{H})$. As a result, $\mathrm{Su}(\mathrm{H})$ is converted from a repressor into an activator, and mediates transcription of the $E(s p l)$ repressors in the nonSOP's (Bailey and Posakony, 1995; Lecourtois and Schweisguth, 1995; Schrons et al., 1992). The $\mathrm{E}(\mathrm{spl})$ repressors then antagonize Ato/ASC and prevent the cells from adopting the default SOP fate (Fig. 4).

\section{Lateral inhibition during eye development:}

Notch signaling is reiteratively employed during eye development. The Drosophila compound eye is composed of $\sim 800$ ommatidia that are precisely arranged in a two dimensional lattice (Fig. 5). Each ommatidium is composed of eight photoreceptor cells (the retinula cells $\mathrm{R} 1-\mathrm{R} 8$ ) and 12 accessory non-neuronal cells such as the cone and pigment cells. Differentiation of the retinal tissue begins in the third instar eye imaginal disc (Fig. 5A), a monolayer of pluripotent neuroepithelial cells. A wave of cell differentiation known as the morphogenetic furrow (MF) sweeps across the eye imaginal disc from its posterior to anterior margin, and during this process specification of the photoreceptor cells occurs (Wolff and Ready, 1991). The R8 photoreceptor is the first cell type to be specified, and is required for the subsequent recruitment of all other cell types into the assembling ommatidia (Fig. 5, (Jarman et al., 1994)). For these reasons, the R8 cells are referred to as the 'founding' photoreceptors. Notch signaling plays biphasic roles during R8 specification (Fig. 5C). Initially, Notch activation induces the expression of the proneural bHLH activator Atonal in the PNC's at the anterior margin of the MF (Fig. 5B,C). This 'proneural enhancement' step is $\mathrm{Su}(\mathrm{H})-$ and $\mathrm{E}(\mathrm{spl})$-independent (Baker et al., 1996). Later, at the posterior margin of the MF, 
Notch mediates the expression of $E(s p l) m 8$, which antagonizes Ato activity, and confines the R8 fate to a single cell from each PNC (Fig. 5). R8 differentiation requires the Ato-dependent expression of the zinc-finger transcription factor Senseless (Sens, (Frankfort et al., 2001; Jafar-Nejad and Bellen, 2004; Nolo et al., 2000; Pepple et al., 2008)). Thus proper R8 selection involves M8-dependent antagonism of both Ato and its target Sens. Consequently, posterior to the MF, R8's emerge in a precise phase shifted pattern, whereby R8's of one column are out of phase with those in the adjacent column. This spacing and patterning is vital for the hexagonal architecture of the adult eye. Consistent with this, a gain of Ato activity results in the specification of excess $\mathrm{R} 8$ cells, an outcome elicited by loss of the $E(s p l) C$ or $S u(H)$. The remaining cells of the cluster are subsequently recruited as secondary photoreceptors. These recruitments are highly ordered and invariant (Fig. 5C). After specification of the $\mathrm{R} 8$ cell, $\mathrm{R} 2 / \mathrm{R} 5, \mathrm{R} 3 / \mathrm{R} 4, \mathrm{R} 1 / \mathrm{R} 6$ photoreceptor pairs are sequentially recruited, with the final being the R7 cell (Cagan and Ready, 1989). Notch-mediated lateral inhibition regulates the specification of all these secondary cell types as well (Cooper and Bray, 1999; Fanto and Mlodzik, 1999; Tomlinson and Struhl, 2001).

\section{Lateral inhibition during bristle development:}

Notch-mediated lateral inhibition is also integral to the development of bristles in Drosophila. The bristles are mechanosensory organs of the peripheral nervous system. The two bristle types that have been extensively studied to explore the mechanisms of neurogenesis are the macrochaetes and the interommatidial bristles (Fig. 6A,B). Both types are positionally invariant and each contains a similar repertoire of cell types (Fig. 6C). These include a socket cell, a sheath cell, a shaft cell and a neuron.

The development of either bristle type depends upon the proper spatial positioning of a cell type that is referred to as the sensory organ precursor (SOP). The SOP, akin to the R8 cell, is also selected from a PNC. The initial formation of the bristle PNCs is independent of 
Notch signaling, and involves the expression of the proneural activators encoded by the Achaete-Scute gene complex in response to pre-pattern factors. The mechanism by which the SOP is selected from the PNC is similar to that for the R8's. In this case, the cell expressing the highest levels of ASC drives activation of Notch in adjacent cells of the PNC, involving lateral inhibition via the $\mathrm{E}(\mathrm{spl}$ ) repressors (Fig. 6D). The events after SOP selection are, however, different from those following R8 specification. The SOP undergoes two asymmetric cell divisions to generate the four distinct cell fates (see above). Specifically, the SOP divides to give rise to the second order precursors, plla and pllb. Asymmetric division of plla generates the external socket and shaft cells. The pllb cell divides to give rise to a glial cell and the third order precursor called plllb. Asymmetric division of the plllb cell gives rise to the two internal cells, the neuron and the sheath cell (Fig. 6).

The reiterated roles played by Notch in SOP selection and in subsequent steps are based upon a body of genetic evidence. For example, loss of Notch or $E(s p l)$ function prior to SOP selection leads to the specification/selection of supernumerary SOP's from each PNC. In the adult, these manifest as ectopic closely spaced bristles (Campos-Ortega, 1998; Skeath and Carroll, 1991). In contrast, the loss of Notch after SOP selection skews the stoichiometric sister cells fates. In this case, multiple phenotypes manifest in the adult, such as split or missing bristles (Bray, 1997; Campos-Ortega, 1997). A split bristle reflects a defect in the asymmetric division of the plla cell, and as a result the socket cell is transformed into a shaft cell. In contrast, a missing bristle can reflect either the loss of SOP, itself, or can be the result of a defect in the asymmetric divisions of SOP. This asymmetric division leads to the specification of two pllb cells, which results in the specification of 'twinned' neuron and sheath cells. Consequently, the shaft and socket cells, which are the external components of this sense organ, are missing.

\section{Regulation of E(spl) repressors and lateral inhibition:}


Given the importance of Notch signaling in neurogenesis significant efforts have been devoted to defining the mechanisms by which $\mathrm{E}(\mathrm{spl})$ proteins mediate neural repression (Giebel and Campos-Ortega, 1997; Ligoxygakis et al., 1998; Nagel and Preiss, 1999). In part, these studies have sought to identify functional domains that are essential for repression. All $\mathrm{E}(\mathrm{spl})$ proteins have in order from the N-terminus, a basic domain for DNA binding, an HLH domain for dimerization, a second HLH called the Orange domain for interaction with Ato/ASC, and a C-terminal WRPW tetrapeptide that is essential for interaction with the corepressor Gro (Fig. 7). While these domains are generally conserved, most of the sequences that underlie isoform-specific differences lie within a region between Orange and WRPW. Interestingly, this region in $\mathrm{E}(\mathrm{spl}) \mathrm{M} 5 / \mathrm{M} 7 / \mathrm{M} 8$ interacts with protein kinase $\mathrm{CK} 2$, and is also necessary for the phosphorylation of these three proteins (Trott et al., 2001b). This interaction and phosphorylation correlate to the presence of a consensus site previously described for CK2 (Trott et al., 2001a). This motif in the case of $E(s p l) M 8$ is $S^{159} D C D$ (Fig. 7). Interestingly, Hes6, the mammalian homologue of $\mathrm{E}(\mathrm{spl}) \mathrm{M} 8$, also harbors a remarkably similar consensus site (Fig. 7) that is also phosphorylated by CK2 (Gratton et al., 2003). Together, these results raise the possibility that phosphorylation by CK2 may regulate Notch signaling.

Follow up studies have been conducted to assess the developmental roles for phosphorylation of $\mathrm{E}(\mathrm{spl}) \mathrm{M} 8$ based upon the observation that misexpression of $\mathrm{E}(\mathrm{spl})$ repressors leads to dominant neural defects. These studies have employed variants of $\mathrm{E}(\mathrm{spl}) \mathrm{M} 8$ that harbor substitutions of Ser ${ }^{159}$, the CK2 phosphoacceptor. These variants are, the non-phosphorylatable isoform M8-S ${ }^{159} \mathrm{~A}(\mathrm{M} 8 \mathrm{SA})$, and the phosphomimetic isoform M8$S^{159} D$ (M8SD). While the overexpression of wild type M8 led to a loss of the interommatidial bristles (IOB's) and the macrochaetes (MC's), it did not elicit any ommatidial defects reflecting the observation that R8 patterning is unaffected. However, overexpression of M8SD elicited a severely reduced eye (Karandikar et al., 2004). This eye phenotype is similar to that elicited by $E(s p l) D$, an allele of $m 8$ that encodes a truncated protein called M8* (Nagel et al., 1999). 
This truncation removes all sequences after the Orange domain, and $M 8^{*}$ is thus deficient for CK2 phosphorylation and Gro-recruitment. In the case of M8SD as well as M8*, the severe reduced eye phenotype reflects a profound loss of R8 cells due to exacerbated interaction with, and antagonism of, Ato. This also reflects in yeast two-hybrid assays, where, the interactions of M8SD and M8* with Ato are of equal potency, whereas that of M8 or M8SA are marginal (Karandikar et al., 2004). Based on these studies, it has been proposed that the Cterminal domain (CtD) of M8, which includes the phosphorylation site, is likely to 'auto inhibit' the Orange domain (Fig. 7). The Orange domain has been previously implicated in the binding of M8 with Ato and Sens (Jafar-Nejad et al., 2003; Jennings et al., 1999). The possibility thus arises that phosphorylation is vital for M8 activity, and that deletion of the CtD in $\mathrm{M}^{*}$ bypasses autoinhibition. If so, phosphorylation by $\mathrm{CK} 2$ becomes central to lateral inhibition.

Unlike the stark differences in the eye, the expression of M8 or M8SD in the bristle lineage elicited loss of MC's with equivalent severity (Karandikar et al., 2004). It was therefore suggested that phosphorylation might play a more predominant role in the eye, but not the bristles. Given that specification of the SOP's and the R8's employ similar mechanisms, the possibility remained open that the absence of a role for CK2 in the bristle reflects a limit phenotype, one not discernible by analysis of ectopically expressed proteins. Therefore, alternative approaches are required to directly establish whether CK2 plays a role in lateral inhibition and, if so, does it operate in both eye and bristle development.

\section{Protein Kinase CK2:}

\section{Overview:}

The Ser/Thr protein kinase CK2 is highly conserved and ubiquitous to eukaryotes. Its targets include proteins involved in gene expression, cell polarity, chromatin structure, cell 
cycle progression, cell signaling, embryogenesis, cell fate determination and development. Consistent with diverse functions, loss of CK2 is lethal in yeast, mammals and Drosophila (Buchou et al., 2003; Jauch et al., 2002; Lin et al., 2002; Padmanabha et al., 1990). CK2 was presumably first identified in the laboratory of Eugene Kennedy in 1954, the first isolation and identification of a protein kinase. They had identified an enzyme, which they called a 'protein phosphokinase', which catalyzed the transfer of the $\mathrm{PO}_{4}$ group of ATP to the model substrate casein (Burnett and Kennedy, 1954). In addition, they demonstrated that this phosphotransfer reaction involved the modification of Ser/Thr residues. This enzyme preparation was named Casein Kinase. However, the relevance of this phosphorylation could not be uncovered since casein is not an in vivo target of CK2, leading Eugene Kennedy to abandon further work on protein kinases. It was not until the seminal work of Krebs and Fisher that the importance of this modification became apparent, work that led to their being awarded the Nobel Prize in 1992. In a perspective article in 1992, titled 'Sailing to Byzantium', Eugene Kennedy comments, "like the base Indian, I cast away a pearl far richer than all my tribe".

In 1955 the seminal works of Edmond Fischer and Edwin Krebs indicated that phosphorylation is required to regulate enzyme activity. They discovered that the enzyme glycogen phosphorylase could be converted from an active to an inactive form via phosphorylation (Fischer and Krebs, 1955). This regulation was catalyzed by the cAMP depended protein kinase (PKA). The activity of this enzyme was, itself, dependent on the second messenger, cAMP. These initial findings have now been extended to virtually all aspects of cell/organismal biology. The enormous impact of this discovery laid the foundation for studies to identify and characterize protein kinases and their roles in diverse cell functions including, cell division, cell polarity, gene expression and animal development.

Casein Kinases were first purified to homogeneity from mammalian tissues in the laboratory of Jolinda Traugh. During this purification, two forms were found and these were named Casein Kinase I and II, based on their order of elution from a phosphocellulose column 
(Hathaway and Traugh, 1979). Following the purification of Casein Kinase II, antibodies that recognized the mammalian enzyme were generated. These antibodies recognized polypeptides of similar molecular mass in tissue extracts from organisms as distant as Drosophila and mammals (Dahmus et al., 1984). These initial findings suggested that this enzyme might well be universally conserved. These efforts enabled the purification of Casein Kinase II from Drosophila, worms, yeast, and plants, and the determination of its subunit composition. Over the years, it became apparent that the name Casein kinase is a misnomer. For this reason, these enzymes were renamed as CK1 and CK2, a nomenclature used henceforth.

\section{Subunits of CK2:}

The CK2 holoenzyme is a tetramer that is composed of two catalytic (CK2 $\alpha)$ and two regulatory subunits (CK2 $\beta)$. The tetrameric conformation of the CK2 holoenzyme is highly conserved from yeast to humans, and is reminiscent of the quaternary structure of Protein kinase A (PKA, (Taylor, 1989)). In the case of PKA, cAMP binds to a central regulatory (R) subunit dimer and triggers the dissociation of the catalytic (C) subunits. This dissociation results in the release of a pseudosubstrate peptide contained within the $\mathrm{R}$ subunit, and a concommitant conformational change that activates the $\mathrm{C}$ subunit. In the case of $\mathrm{CK} 2$, however, dissociation of the tetramer does not appear to be a regulatory mechanism. Purified CK2 maintains its tetrameric conformation even in the presence of $1 \mathrm{M} \mathrm{NaCl}$, and efforts to identify a second messenger that elicits dissociation did not bear fruit. Cochet and Chambaz used resolution and reconstitution to address this issue. Using urea and guanidine hydrochloride, they isolated individual subunits and found that the monomeric CK2 $\alpha$ subunit displayed catalytic activity, which was approximately $25 \%$ of that of the tetramer (Cochet and Chambaz, 1983). Furthermore, the addition of renatured CK2 $\beta$ fully reconstituted the 
tetrameric state and catalytic activity. These studies, since confirmed by recombinant proteins, established that even if a novel second messenger were to trigger tetramer dissociation, it could only alter activity by a factor of four (Bidwai et al., 1992; Birnbaum et al., 1992; Boldyreff et al., 1993; Lin et al., 1991). This is in contrast to PKA where the tetramer displays no discernable kinase activity. Consequently, CK2 is now generally considered to be a second messenger independent kinase.

\section{Biochemistry of CK2:}

When compared to other kinases, CK2 can phosphorylate Ser/Thr residues using either ATP or GTP as a phosphoryl donor (Dahmus et al., 1984; Glover et al., 1983; Hathaway et al., 1980; Meggio et al., 1982). In addition, CK2 $\alpha$ also catalyzes the autophosphorylation of CK2 $\beta$. Unlike other kinases, however, autophosphorylation of CK2 $\beta$ does not appear to regulate subcellular localization, susceptibility to proteolysis or activity of CK2 (Meggio et al., 1983; Meggio and Pinna, 1984). However, genetic evidence from Drosophila (see below) indicates that this reaction is essential for CK2 $\beta$ functions in vivo (Jauch et al., 2002). Using synthetic peptides, the laboratory of Edwin Krebs described a consensus for substrate recognition and phosphorylation. This consensus is $(S / T)-(D / E)-(X)-(D / E)$, in which the acidic residues at positions $n+1$ and $n+3$ are rate limiting for phosphorylation (Kuenzel and Krebs, 1985; Kuenzel et al., 1987). This consensus site is unique to CK2, and indicates that this enzyme preferentially phosphorylates target proteins at Ser/Thr residues in acidic microdomains. In addition, CK2 can function as part of hierarchical phosphorylation cascades as pSer or pThr biochemically mimics the effect of acidic residues.

\section{Structure of CK2:}

X-ray crystallographic analyses of CK2 have provided novel insights into the biochemical properties of CK2. The comparison of structure of CK2 holoenzyme from various 
organisms represents a typical $\alpha_{2} \beta_{2}$ quaternary structure (Chantalat et al., 1999; Niefind et al., 2001; Niefind et al., 1998; Rasmussen et al., 2005). In this case (Fig. 8), a central dimer of CK2 $\beta$ is stabilized through a $\mathrm{Zn}$ finger domain, which is similar to that in aspartate transcarbamylase. Accordingly, CK2 $\beta$ dimerization mutants fail to interact with CK2 $\alpha$ indicating that dimerization of $\beta$ subunits is required for association of the catalytic subunits (Kusk et al., 1995). The catalytic subunits are thus assembled on the core $\beta-\beta$ dimer. In the holoenzyme the individual catalytic subunits do not contact each other and their active sites project outwards (Niefind et al., 2001). Unexpectedly, the N-terminal autophosphorylation site of CK2 $\beta$ is distal to the catalytic sites, indicating that autophosphorylation must occur via intermolecular interactions between CK2 tetramers.

The structure of CK2 $\alpha$ subunit is comprised of a bilobed structure that is prototypical of Ser/Thr protein kinases. In the case of CK2, an N-terminal domain is rich in beta sheets, whereas the C-terminal domain is primarily alpha helical. The interface between these two domains is the active site. Structurally, $\mathrm{CK} 2 \alpha$ is stabilized via interactions between its $\mathrm{N}$ terminal region with the activation segment and a cluster of highly conserved basic residues of the substrate recognition region (Hanks and Quinn, 1991; Niefind et al., 1998). This strong attachment of the $\mathrm{N}$-terminus with the activation segment is found in both the isolated catalytic subunit and that bound to CK2 $\beta$ supporting the constitutive activity of monomeric CK2 $\alpha$. The crystal structure of $\mathrm{CK} 2 \alpha$ also reveals that the nucleotide-binding site is unusually wide, enabling this protein to utilize ATP and GTP as phosphoryl donors (Niefind et al., 1998). In the tetrameric state, however, the C-terminal region of CK2 $\beta$ stabilizes CK2 $\alpha$, thereby enhancing catalytic activity (Chantalat et al., 1999; Niefind et al., 2001).

\section{CK2 and Cell Biology:}

Earlier research on CK2 focused on its potential role as a regulator of cell cycle 
progression. It was initially observed that stimulation of mammalian cells in culture with the mitogen Epidermal Growth Factor (EGF) elicited oscillations in the levels and activity of CK2 (Sommercorn et al., 1987). Given the role of EGF in inducing cell division, the possibility arose that this kinase is involved in the cell cycle. Moreover, it was found that CK2 activity oscillated in synchrony with the G1/S and G2/M phases of the cell cycle. A direct evidence for the role of CK2 was identified by the observation that deletion of the genes encoding the catalytic subunits of yeast CK2 resulted in a 'pseudomycelial' morphology (Padmanabha et al., 1990). This phenotype is a hallmark of the uncoupling of cell growth from cell division in budding yeast, and has been well described for bona fide cell cycle regulators such as cdc2/28. The isolation of temperature sensitive alleles of yeast CK2 provided the first direct demonstration that the activity of this enzyme is essential for the G1/S and G2/M transitions (Hanna et al., 1995; Rethinaswamy et al., 1998). Additional findings also implicate CK2 in tumorigenesis. Perhaps the most illustrative finding, and one that may be unique, is the molecular mechanism for a fatal leukemia that manifests in cattle. This disease, called Theileriosis, is characterized by hyperproliferation of T and B-lymphocytes. Remarkably, Onesmo K. ole-MoiYoi, a veterenian in Kenya made the observation that cattle displaying symptoms of this disease invariably harbored the parasitic protozoa, Theileria parva, in their circulatory system. He reasoned that if this parasite triggers the leukemia, it should respond to anti-parasitic drugs. His findings were, indeed, the case. He subsequently demonstrated that this parasite preferentially infects B and T cells, and during this stage expresses a unique secretory form of CK2, which thus ends up in the cytosol of the host cell (ole-MoiYoi, 1995; ole-MoiYoi et al., 1993). Consistent with the conservation of structure of CK2, the parasitederived enzyme likely deregulates endogenous control over the cell cycle. To our knowledge, this is the only case of a parasite-induced leukemia. Later studies using normal human and cancer cells have, in essence, corroborated these findings.

Consistent with a role in the cell cycle, CK2 has been shown to associate with mitotic 
spindles and the centrosomal apparatus (Yu et al., 1991). In addition, CK2 interacts with the peptidyl-prolyl isomerase Pin1, a vital regulator of cell division and replication checkpoint control (Winkler et al., 2000), and targets a number of proto-oncogene derived proteins such as Myc, Myb, Fos and Jun (Luscher et al., 1989).

Given the vital role for CK2 in cell cycle regulation and its implications in cancer, the characterization of its targets took on greater importance. With the availability of genome sequences and techniques for high throughput proteomics, a staggering number of CK2 targets have been identified. These include proteins involved in multiple cellular functions such as DNA replication, transcription, translation, cytoskeleton architecture, cell polarity and development (reviewed in Meggio and Pinna, 2003; Pinna, 2002). Unfortunately, our knowledege of the consequences of these phosphorylation events on the target proteins and their biological consequences in vivo are still, woefully, inadequate.

\section{Molecular genetic studies of CK2:}

Since chapter 4 of this dissertation involves analysis of Drosophila CK2 conditional alleles screened in a yeast-based bioassay, this section briefly describes salient features of Saccharomyces cerevisiae CK2. In yeast, the CKA1 and CKA2 genes encode for the catalytic $\alpha$ and $\alpha$ ' subunits, respectively, whereas CKB1 and CKB2 encode the regulatory $\beta$ and $\beta$ ' subunits (Bidwai et al., 1994; Bidwai et al., 1995; Chen-Wu et al., 1988; Padmanabha et al., 1990; Reed et al., 1994). The simultaneous deletion of CKA1 and CKA2 genes is lethal. This lethality is fully rescued by the expression of the catalytic subunit of Drosophila CK2 (Bidwai et al., 1992). This rescue formed the basis for an in vivo bioassay for putative ts alleles of Drosophila CK2 (Kuntamalla et al., 2009).

\section{Drosophila CK2:}

In Drosophila, a single CK2 $\alpha$ gene located on third chromosome encodes for the 
catalytic subunit, whereas the regulatory subunit is encoded by $C K 2 \beta$ on the $\mathrm{X}$ chromosome (Bidwai et al., 2000; Saxena et al., 1987). The CK2 $\alpha$ gene is comprised of two alternative 5' untranslated exons and a single large uninterrupted exon that encodes the complete open reading frame. In contrast, the open reading frame in $C K 2 \beta$ is contained within six exons and is further complicated by the presence of five 5' untranslated exons (Bidwai et al., 2000). The in vivo functions of these CK2 $\beta$ derived alternative transcripts are currently unknown.

To date, two mutants of $C K 2 \alpha$ have been identified based on their ability to perturb the circadian clock. These are Timekeeper (Tik), and its partial revertant, called Tik ${ }^{R}$ (Lin et al., 2002). The former allele harbors two substitutions, $M^{161} K$ and $E^{165} D$. The $M^{161} K$ substitution is located within the ATP-binding pocket, and thus inactivates CK2 (Niefind et al., 2001; Rasmussen et al., 2005). The $E^{165} \mathrm{D}$ substitution was thought to be silent because it involved a conservative replacement, but studies indicate that this substitution does affect CK2 functions. This substitution lies in a highly conserved sequence motif, $H E^{165} \mathrm{NRKL}$, which mediates the interaction of human CK2 $\alpha$ with the phosphatase PP2A (Heriche et al., 1997). Studies in Drosophila, now suggest that the interaction of CK2 $\alpha$ with PP2A may downregulate phosphatase activity. It has therefore, been suggested that Tik maybe a 'double hit' (KunttasTatli et al., 2009). On one hand, it inactivates CK2 activity and, on the other, it leads to enhanced PP2A activity.

The revertant allele, $T_{i k}{ }^{R}$ harbors an $\mathrm{R}^{242} \mathrm{G}$ substitution and a deletion of seven internal residues (234-240), in addition to the two mutations originally seen in Tik. Recombinant Tik ${ }^{R}$ protein appears insoluble, indicating defects in protein folding (Lin et al., 2002). Moreover, it has been shown that unlike wild type CK2 $\alpha$ or Tik, the TikR protein is deficient for physical interaction with CK2 $\beta$ (Kunttas-Tatli et al., 2009). This interaction is essential for assembly of the tetrameric holoenzyme. Therefore, Tik is competent to integrate into and 'poison' the endogenous holoenzyme. In contrast, TikR is excluded from the holoenzyme, and its 
revertant properties may reflect the possibility that halved CK2 activity/dosage (in TikR/+ animals) may still be sufficient for the circadian clock.

While Tik/+ heterozygous flies exhibit severe clock defects those for TikR/+ animals are more modest. Given the underlying lesions, however, Tik or TikR are both lethal when homozygous, and the effective lethal phase manifests at the first larval instar (Lin et al., 2002). Thus the maternal contribution of CK2 is likely to be sufficient for the completion of embryogenesis. Neither allele in the heterozygous state, however, displays overt developmental abnormalities, indicating that the residual levels of CK2 are sufficient. Analysis in the homozygous state is precluded because lethality preceeds early eye or bristle development. This neccessitates alternative approaches, such as RNAi or ts-alleles to dissect the roles of CK2.

Recently, another allele of CK2, CK2 $2^{M B 00477}$ has been described that harbors a transposable minos element in the 5' UTR (Bellen et al., 2004; Metaxakis et al., 2005). Unlike Tik or Tik ${ }^{R}, C K 2^{M B 00477}$ homozygotes complete the three larval stages and lethality manifests at the pupal stage. This indicates that $C K 2^{M B 00477}$ is a hypomorph. Consistent with this possibility, eye and bristle development is normal in $C K 2^{M B 00477} /+$ heterozygotes. Studies described in chapter 4 indicate that flies homozygous for $C K 2^{M B 00477}$ display unique retinal defects that are characteristics of loss of Notch functions.

Clock defects have also been found with an allele of $C K 2 \beta$, andante. Homozygous andante flies are viable, indicating that this is a hypomorphic allele (Akten et al., 2003). In the case of andante, the mutant protein has been proposed to be deficient in stable formation of the holoenzyme. However, analysis of a human variant of andante (Rasmussen et al., 2005) argues against this possibility, and the mechanism underlying its clock defect in Drosophila remains unresolved. Analysis of $C K 2 \beta^{\text {mbuP1 }}$, a null allele, during eye or bristle development is rendered difficult as it is embryonic lethal (Jauch et al., 2002). 


\section{Regulation of CK2:}

The regulation of CK2 remains unresolved. Unlike other kinases, CK2 appears to not be responsive to second messengers and there is no evidence that phosphorylation plays a role analogous to that in other kinases such as PKC, PKA and components of the RTK pathway (reviewed in Litchfield, 2003). CK2 expression during embryogenesis or eye development is not spatially regulated (Jaffe et al., 1997; Karandikar et al., 2004), indicating that alternative mechanisms of regulation must exist.

The possibility that $\mathrm{CK} 2$ is operating as part of a supramolecular complex remains open (Giot et al., 2003; Goldstein and Lampen, 1976). The roles of this kinase in cell cycle progression, embryogenesis and the circadian clock would indicate that it is precisely controlled in vivo. CK2 and PP2A are known to function antagonistically during phosphorylation of the central clock protein Period (PER, (Sathyanarayanan et al., 2004)). These studies raise the possibility that CK2 and PP2A activities are coordinated via complex formation. Such a regulated complex may exert control over repression by E(spl)M8 during eye and bristle development. Studies in chapter 3 (see below) are aimed at addressing this issue.

\section{Protein Phosphatase PP2A:}

\section{Overview:}

Reversible phosphorylation alters protein activity by modulating conformation, cellular locale and interaction with other proteins, reasons for which this regulation is integral for functioning of protein networks. Based on these fundamental requirements, reversible phosphorylation is integral to a wide range of cellular processes. These include gene regulation, cell cycle progression, metabolic control, cytoskeletal organization, cell adhesion, cellular transport and secretion. These diverse requirements are underscored by the fact that 
the human genome encodes in excess of 500 distinct kinase and phosphatase genes (reviewed in Barford et al., 1998; Eichhorn et al., 2009; Shi, 2009; Virshup and Shenolikar, 2009)).

Eukaryotic protein phosphatases are broadly classified into Ser/Thr phosphatases (STPs) and the protein tyrosine phosphatases (PTPs). The STP's are divided into two distinct families, phosphoprotein phosphatases (PPP) and the $\mathrm{Mg}^{2+} / \mathrm{Mn}^{2+}$ dependent phosphatases (PPM). Within the PPP's, there are three sub-families, PP1, PP2A and PP2B also called Calcineurin (Denu et al., 1996). Among them, PP1 and PP2A are the most abundant and are involved in diverse cellular functions. Based on functional diversity and abundance, it is expected that a large number of distinct catalytic subunits would be required for their function. However, in humans only eleven genes encode for the catalytic subunits of PP1 and PP2A. This is in contrast to over 400 genes in humans encoding for distinct kinase catalytic subunits (reviewed in Virshup and Shenolikar, 2009). Moreover, in vitro the phosphatase catalytic subunits are known to dephosphorylate substrates in a somewhat non-specific manner. How then does the substrate specificity and functional diversity of these phosphatases arise? It appears to be the case that in the holoenzyme conformation of the PPP's, the catalytic subunits associate with a variety of regulatory subunits, which either promote or antagonize target protein recognition. The functional diversity thus stems from numerous regulatory subunits that are involved in determination of substrate specificity, subcellular localization and activity of phosphatase holoenzyme (reviewed in Virshup and Shenolikar, 2009).

\section{Subunits of PP2A:}

The PP2A holoenzyme (Fig. 9) is a heterotrimer that consists of a catalytic, scaffolding and regulatory subunit. The PP2A core dimer is comprised of a $36 \mathrm{kD}$ catalytic subunit (C) and $65 \mathrm{kD}$ scaffolding subunit (A or PR65) subunit. In vivo, the core (AC) dimer associates with various regulatory subunits to form the trimeric holoenzyme (reviewed in Janssens and 
Goris, 2001). Two isoforms ( $\alpha$ and $\beta$ ) for the $C$ and $A$-subunits have been identified in mammalian cells. Among these, the $\alpha$ isoform of the core enzyme is more abundant. The complexity of enzyme subtypes predominantly reflects diversity of the regulatory subunits. These have been grouped into four families, B/PR55, B'/PR61, B'/PR72 and B'”/PR93, and each family is encoded by multiple genes (reviewed in Goldberg, 1999). While the PP2A core dimer displays constitutive and ubiquitous expression, it is the regulatory subunits that exhibit specific spatial/temporal expression, thereby promoting the dephosphorylation of one group of proteins and at the same time inhibiting that of the others (McCright, 1996; Strack et al., 1998). Studies in Drosophila S2 cells indicate that targeted knockdown of all of the four regulatory subunits results in the ablation of the $A$ and $C$ subunits, indicating that the heterotrimeric form is required to stabilize PP2A in vivo (Li et al., 2002; Silverstein et al., 2002).

\section{Structure of PP2A:}

Even though the subunits of PP2A were cloned 20 yrs ago, difficulties in expression, purification and crystallization of PP2A subunits hindered an understanding of its structure. Recently, the structures of the PP2A core dimer and holoenzyme have been solved (Cho and Xu, 2007; Xing et al., 2006; Xu et al., 2006), and these have provided important mechanistic insights on structure and function of PP2A.

The PP2A C-subunit is structurally similar to other members of the PPP family. The similarity lies in the characteristic $\alpha / \beta$ folds and presence of two metal ions $\left(\mathrm{Mn}^{2+}\right)$ in the active site. The scaffolding PP2A-A subunit is comprised of 15 HEAT (Huntington Elongation factorPP2A A subunit-TOR kinase) repeats. Each HEAT repeat consists of a pair of antiparallel $\alpha$ helices, whose overall shape resembles a horseshoe (Xing et al., 2006; Xu et al., 2006). It is within this horseshoe shaped protein that the catalytic and regulatory subunits associate to 
generate the functional holoenzyme. Importantly, the catalytic subunit on its own or as an AC dimer displays a rather shallow active site, which is thought to underlie weak and non-specific activity. It appears that the regulatory subunit may provide a binding interface for target proteins and their presentation to the active site of the C-subunit (reviewed in Mumby, 2007). This possibility underscores the paradoxical observations that the regulatory subunits can facilitate the recognition of one protein and at the same time inhibit that of others. Expectedly, this bimodal activity of the regulatory subunits has complicated biochemical and genetic studies.

\section{Drosophila PP2A:}

Molecular genetic studies in Drosophila have uncovered roles for PP2A in cell cycle regulation, development and signaling pathways. Unlike mammals, in Drosophila single copy genes encode PP2A-C and PP2A-A subunits. However, four genes encode for the PP2A regulatory subunits. The B/PR55 subunit is encoded by twins (tws), B'/B56 is encoded by two genes B56-1 and widerborst (wdb), and B"'PR72 is encoded by CG4733. This is simple as compared to the multiple genes in mammals that encode for each of the regulatory subunits (reviewed in Janssens and Goris, 2001).

Initial molecular genetic studies with the catalytic and distinct regulatory subunits of PP2A uncovered its role in MAPK signaling. Studies in Drosophila eye development indicated that mutants of $m t s$ enhanced the rough eye phenotype of a constitutively active variant of Ras1, the effector of RTK signaling (Wassarman et al., 1996). In addition, duplications in the wing imaginal disc and altered cell fate lineage in mechanosensory organs are associated with the mutant alleles aar1 (abnormal anaphase resolution) and twins $P$, both affecting the gene encoding the PR55 subunit (Shiomi et al., 1994; Uemura et al., 1993). Analysis of twins mutants also uncovered its requirement in the wingless signaling pathway, where it plays a role in the stabilization of armadillo/ $\beta$-catenin, the terminal component of wingless signaling 
(Bajpai et al., 2004). Genetic analysis of the PP2A-B' (PR-61) regulatory subunit wdb revealed its role in planar cell polarization and the specification of mechanosensory organs (Abdelilah-Seyfried et al., 2000; Hannus et al., 2002). PP2A-B' also interacts with Hox protein SCR (Sex comb reduced) and loss of B' results in loss of salivary glands, a phenotype mimicked by SCR null mutants (Berry and Gehring, 2000). Recent genetic studies report a requirement for $\mathrm{Wdb}$ in the regulation of the $\mathrm{Zn}$-finger transcription factor Cubitus interruptus (Ci), a crucial component of Hedgehog signaling (Jia et al., 2009). In summary, molecular and genetic studies of PP2A-B and B' regulatory subunits in vivo have revealed a number of developmental processes regulated by PP2A.

\section{PP2A and MAPK:}

The identification of PP2A as a regulator of MAPK signaling was first identified through studies in Drosophila photoreceptor development. Expression of a constitutively active form of Ras1 (Ras $1^{\text {Val12 }}$ ) results in transformation of the non-neuronal cone cells to R7 photoreceptors. The resulting rough eye phenotype is enhanced by loss of function alleles of the PP2A-C subunit (Wassarman et al., 1996). Again, in the same study, PP2A-C subunit alleles suppressed the rough eye phenotype of a constitutively active form of the downstream Ser/Thr kinase Raf, suggesting that PP2A regulates MAPK signaling both positively and negatively (Wassarman et al., 1996). It was concluded that PP2A might execute these opposite effects via different regulatory subunits. Studies in cultured cells demonstrate that the PP2A-B regulatory subunits, PR55 $\alpha$ and PR55 $\delta$ positively regulate MAPK signaling by dephosphorylating RAF-1 and kinase suppressor of RAS (KSR1), a protein that so far appears to be unique to Drosophila. In contrast, the PP2A B' regulatory subunits, PR61 $\beta$ and PR61 $\gamma$ negatively regulate MAPK signaling by directly dephosphorylating extracellular regulated kinase (ERK), the terminal effector of MAPK signaling (reviewed in Eichhorn et al., 2009; Junttila et al., 2008). The possibility that PP2A may target the $E(s p l)$ proteins to oppose 
the activating effects of CK2 is the subject of investigations described in chapter 3.

\section{Scope of Dissertation:}

This dissertation describes studies to directly demonstrate a role for CK2 in lateral inhibition (chapter 2, (Bose et al., 2006)), define the genetic interactions between alleles of CK2, PP2A, Notch and E(spl) during sense organ and wing morphogenesis (chapter 3 ), and conclude with studies on the development and initial characterization of flies rescued with putative ts-alleles of CK2 $\alpha$ (chapter 4). 


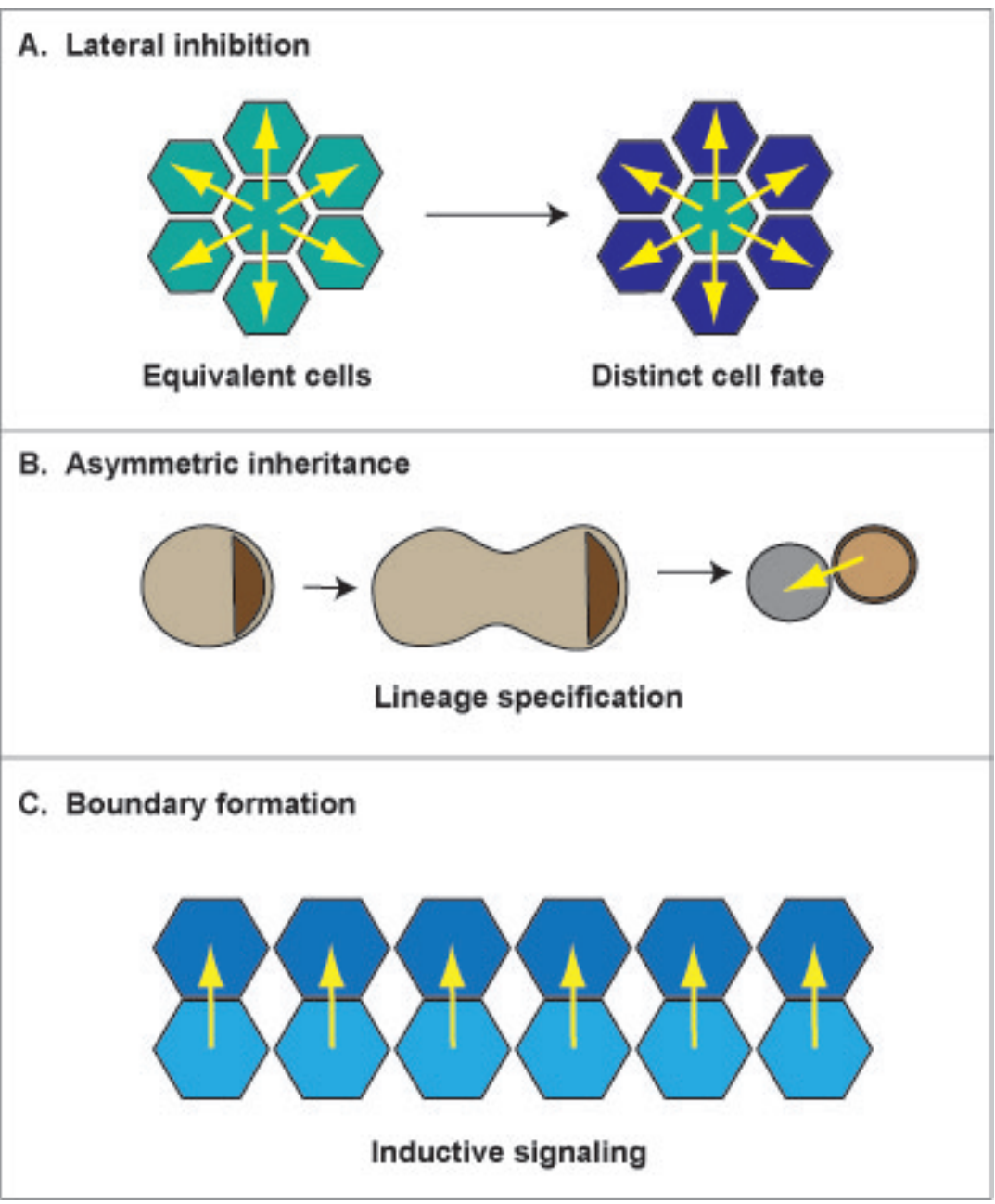

Fig. 1. The different modes of Notch function in animal development. (A) Lateral inhibition: A group of equivalent cells acquire distinct cell fates via inhibitory Notch signaling. (B) Lineage specification: Asymmetric inheritance of a Notch regulator denoted in brown. Two distinct cell types are formed. (C) Inductive signaling: Notch signaling between two nonequivalent population of cells lead to segregation into two distinct cell fates. Yellow arrows denote the direction of Notch signaling. (Adapted from Bray, 2006). 


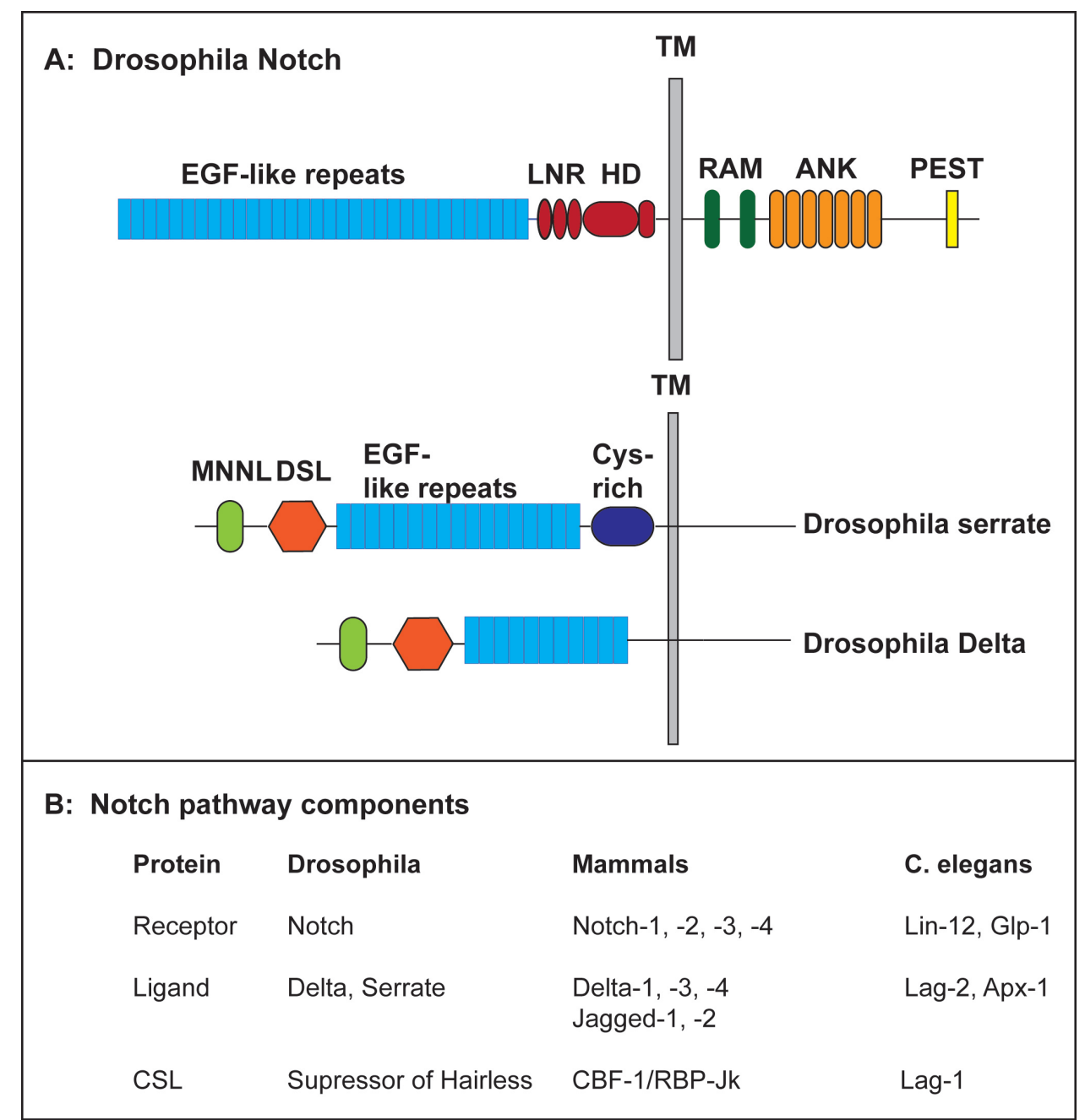

Fig. 2. Notch signaling pathway- Drosophila Notch receptor and the core components. (A) Domain organizations in Drosophila Notch and the ligands Serrate and Delta. (B) The names of the core components in different species. (Adapted from Gordon et al., 2008; Lai, 2004) 


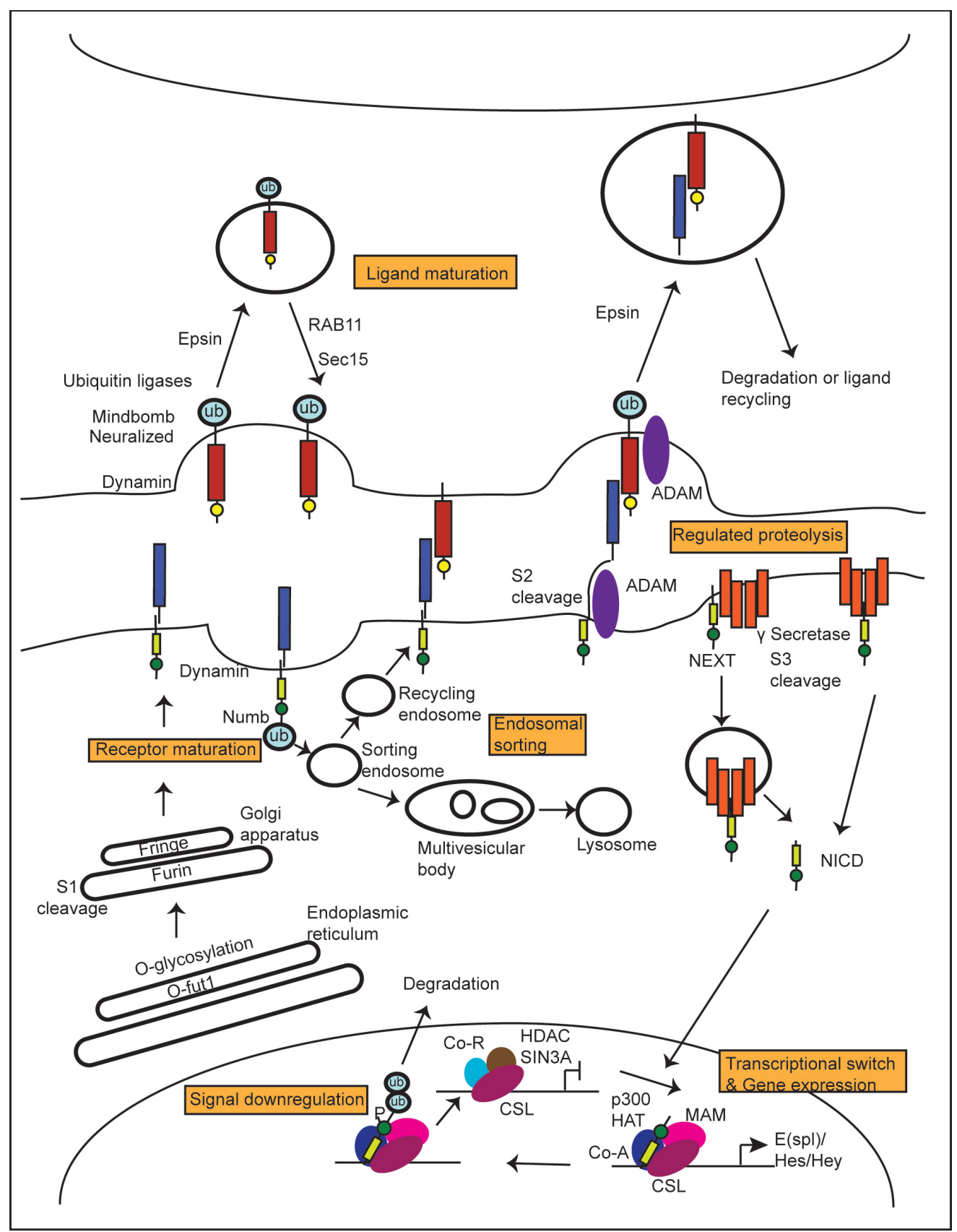

Fig. 3. Notch signaling pathway- a schematic representation of the regulatory mechanisms. The primary steps that regulate Notch signaling pathway are receptor maturation, endosomal sorting, ligand maturation, regulated proteolysis, transcriptional switch and gene expression and signal down regulation. (Adapted from llagan and Kopan, 2007). 


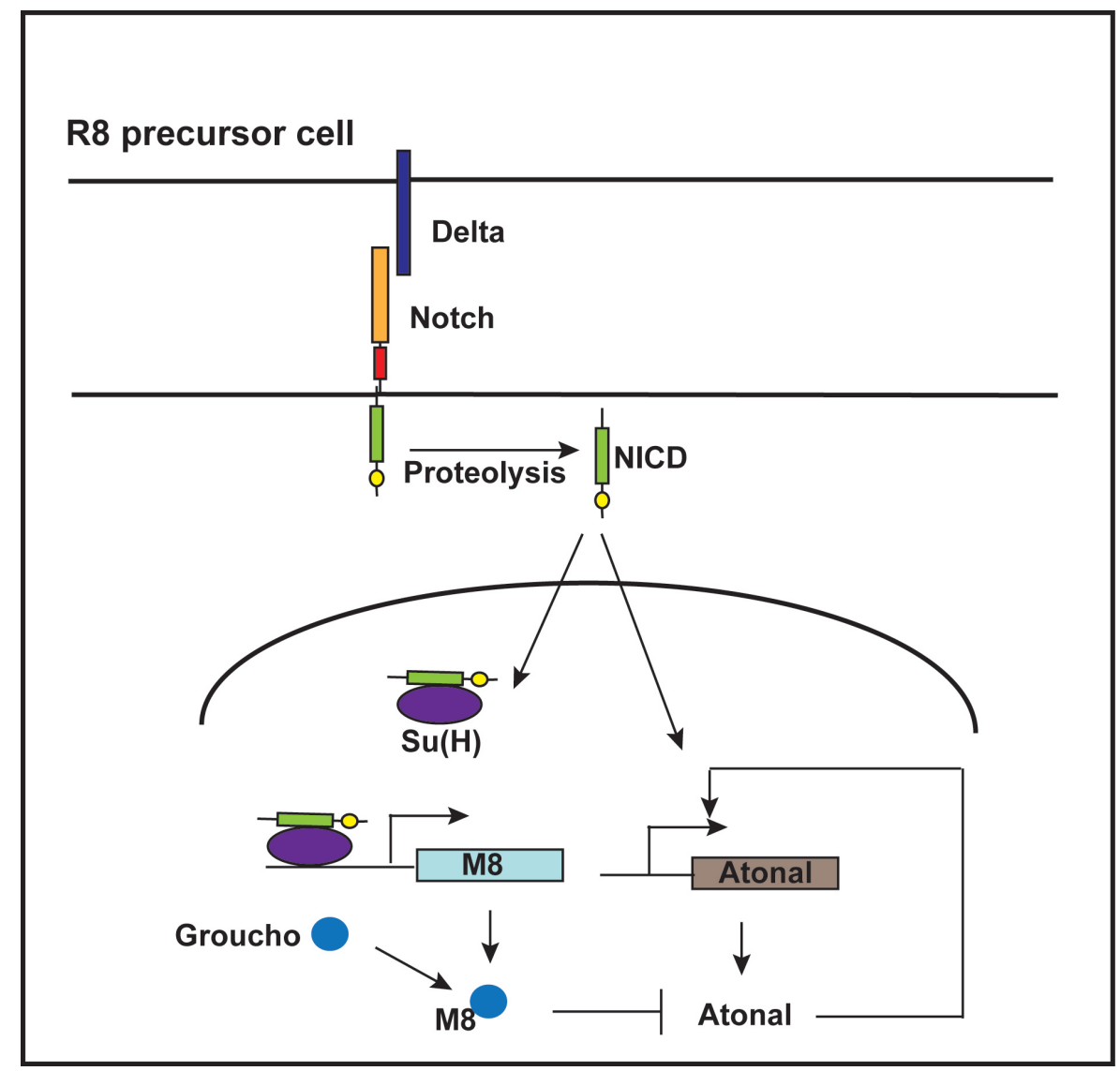

Fig. 4. Representation of Notch-mediated lateral inhibition during Drosophila R8 specification.

Notch signaling terminates with the expression of the $\mathrm{E}(\mathrm{spl})$ repressors (here $\mathrm{M} 8$ is indicated) that along with the co-repressor Groucho antagonizes the proneural protein Atonal. Atonal expression is regulated by the positive feed back loop. 


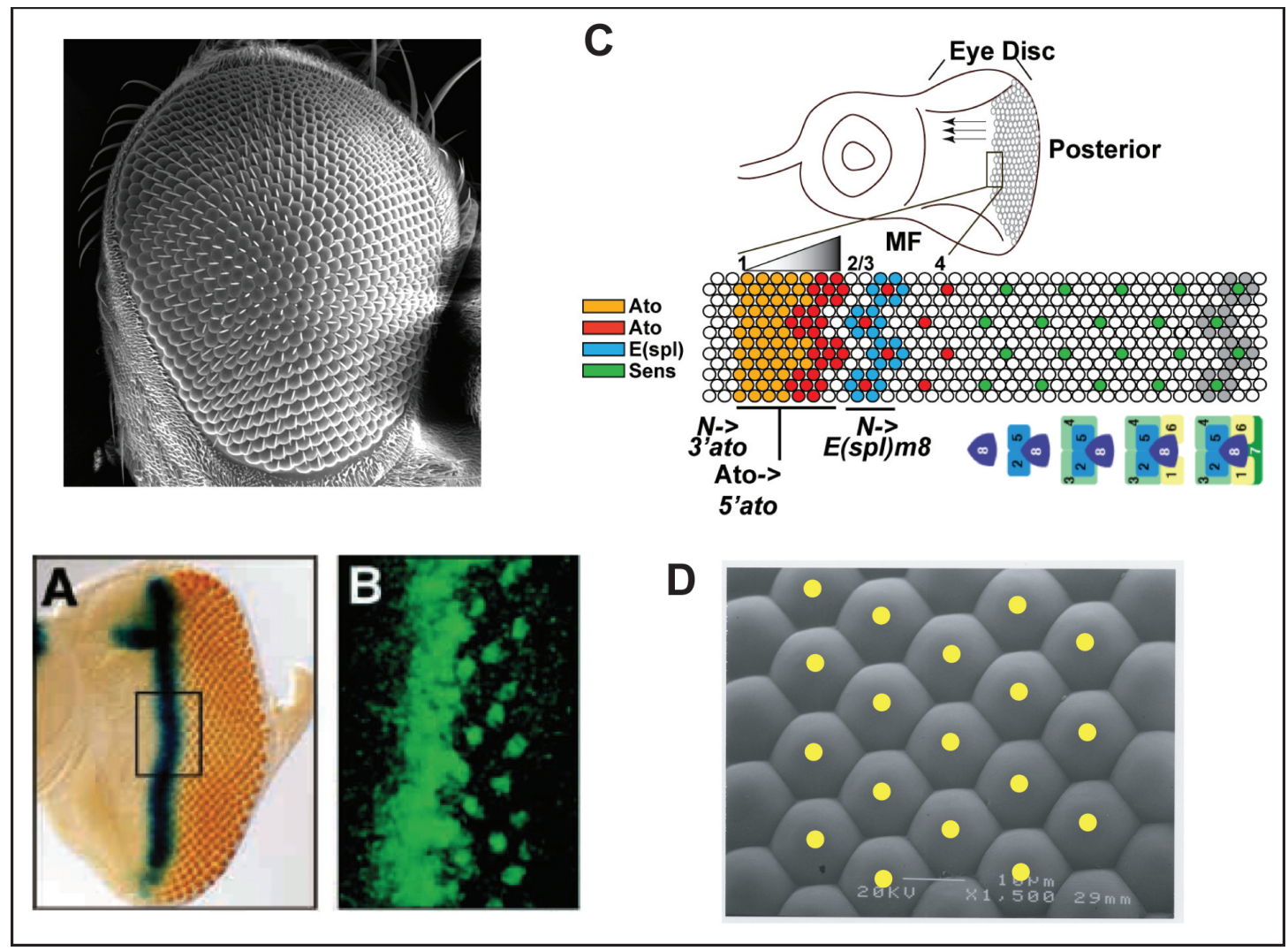

Fig. 5. R8 specification and retinal patterning in the Drosophila compound eye. (A)

Morphogenetic furrow (MF, blue vertical stripe) progresses from the posterior to the anterior of the eye disc. (B) Expression of the proneural protein Atonal in the eye disc. Atonal expression is ubiquitous in the anterior region of the MF and posteriorly resolves into single cells that become R8's. (C) A schematic representation of biphasic Notch signaling during R8 specification. In the first phase Notch induces atonal expression anterior to the MF and in the second phase (in MF) activates $m 8$ expression that inhibits atonal. Inset below represents sequential recruitment of secondary photoreceptors following R8 specification. (D) The hexagonal array of ommatidia in the adult compound eye. The yellow dots represent individual facets. (Adapted from Frankfort and Mardon, 2002) 


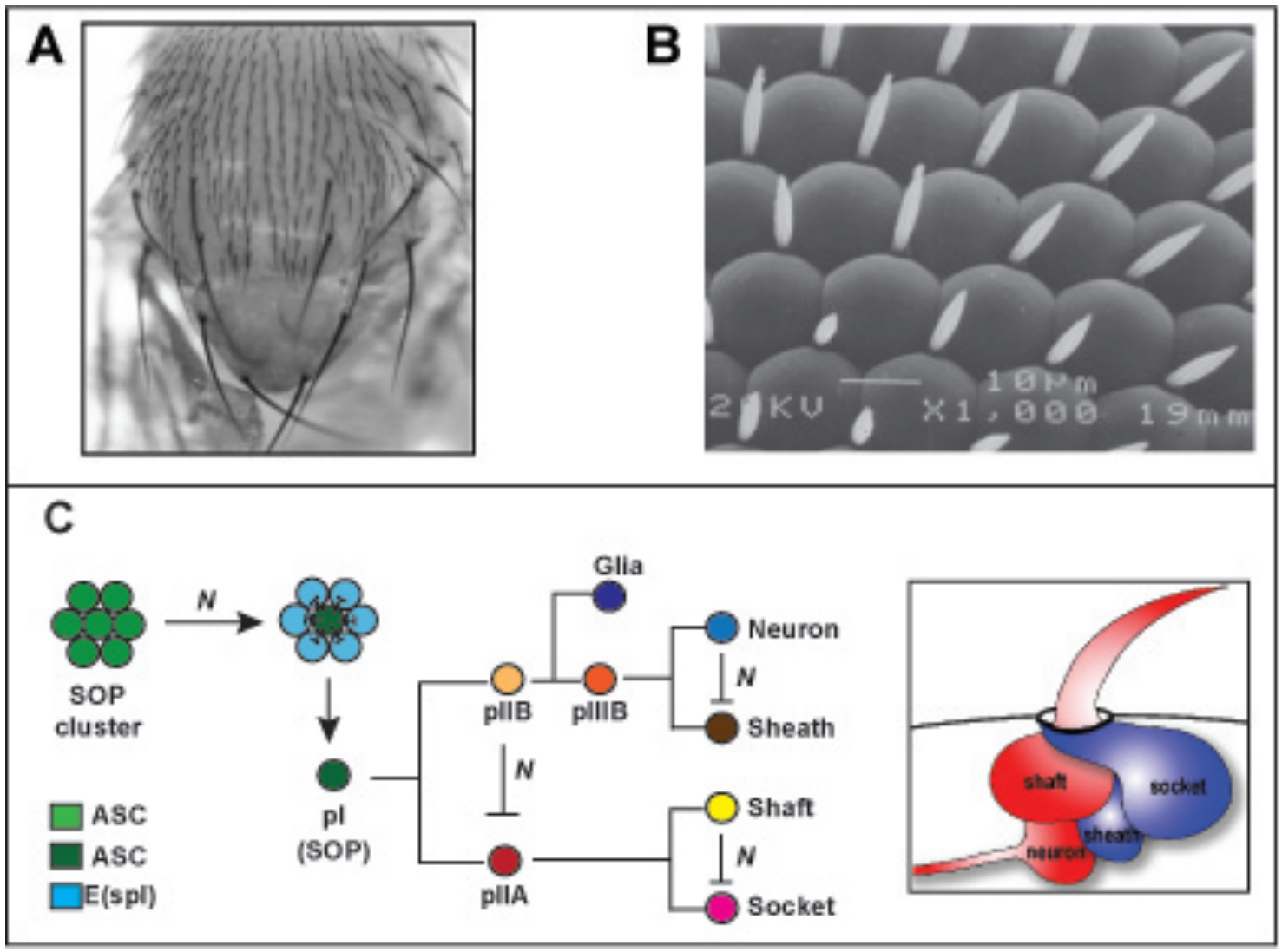

Fig. 6. Patterning and morphogenesis of the Drosophila bristle mechanosensory organs. (A) The Macrochaetes (MC's) and the microchaetes (mc's) on the nota. (B) The interommatidial bristles (IOB's). (C) Lateral inhibition during SOP selection in the neuroectoderm and the SOP lineage specification. Inset, the four different cell types that constitute a bristle. 


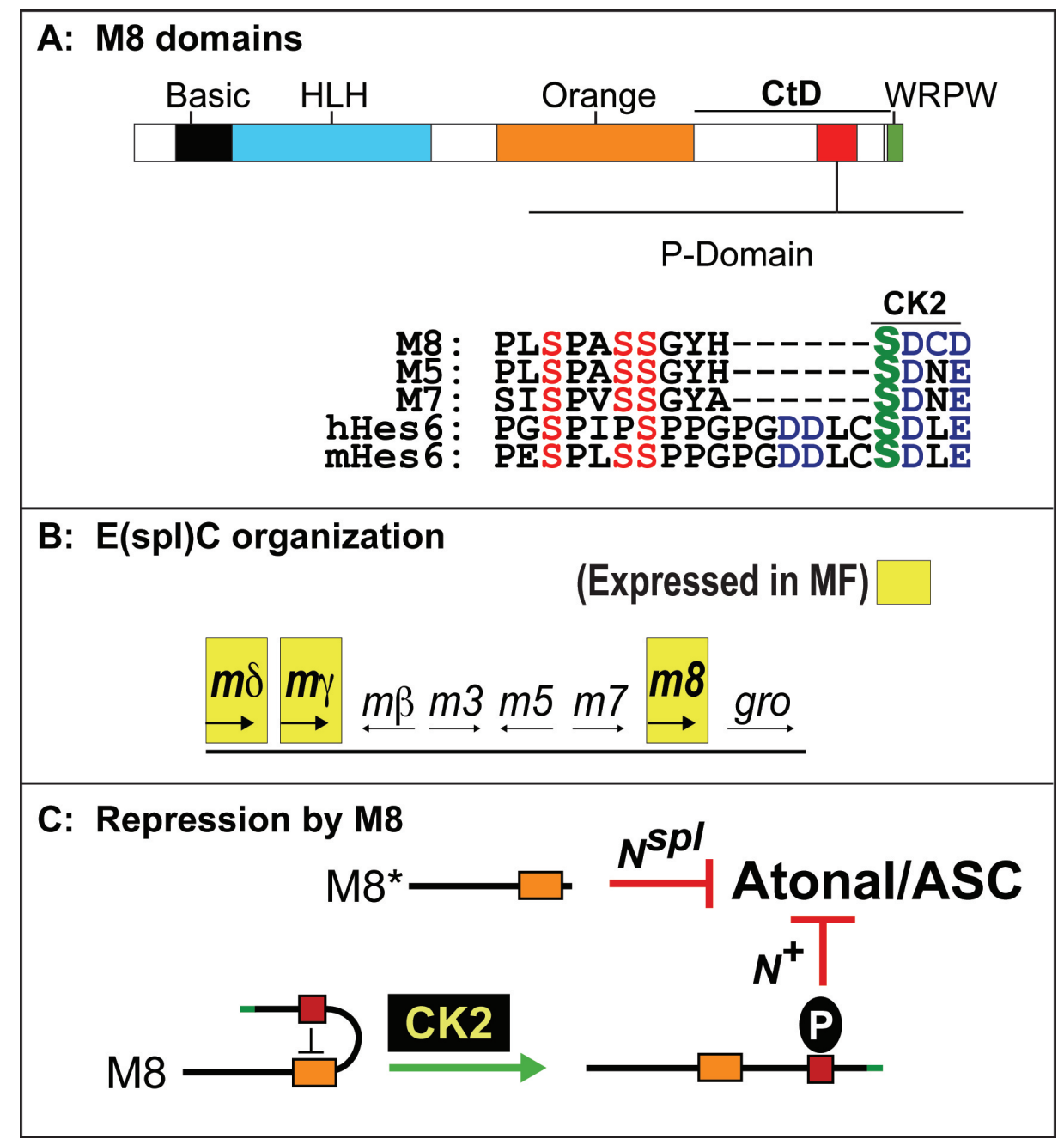

Fig. 7. Domain organization and regulation of $E(s p l)$ M8. (A) The schematic of the functional domains of $\mathrm{E}(\mathrm{spl}) \mathrm{M} 8$. The C-terminal domain (CtD) harbors the conserved phosphorylation domain (Pdomain). The sequence alignment of the P-domain in M8, M5, M7 and human and murine Hes6 shows the highly conserved CK2 phosphorylation site (Serine in green) and additional conserved Serine residues (in red). (B) The third chromosome (96F8-96F10) Enhancer of split complex $(E(s p l) C)$ encodes for functional proteins, the $\mathrm{E}(\mathrm{spl})$ repressors and co-repressor Groucho. $E(s p l) m \delta, m \gamma$ and $m 8$ are expressed in the MF (yellow boxes). (C) The Autoinhibition model. Phosphorylation of M8 by CK2 regulates antagonism of Atonal/ASC in $N^{+}$flies, whereas $M 8^{*}$ requires $N^{\text {spl }}$. 


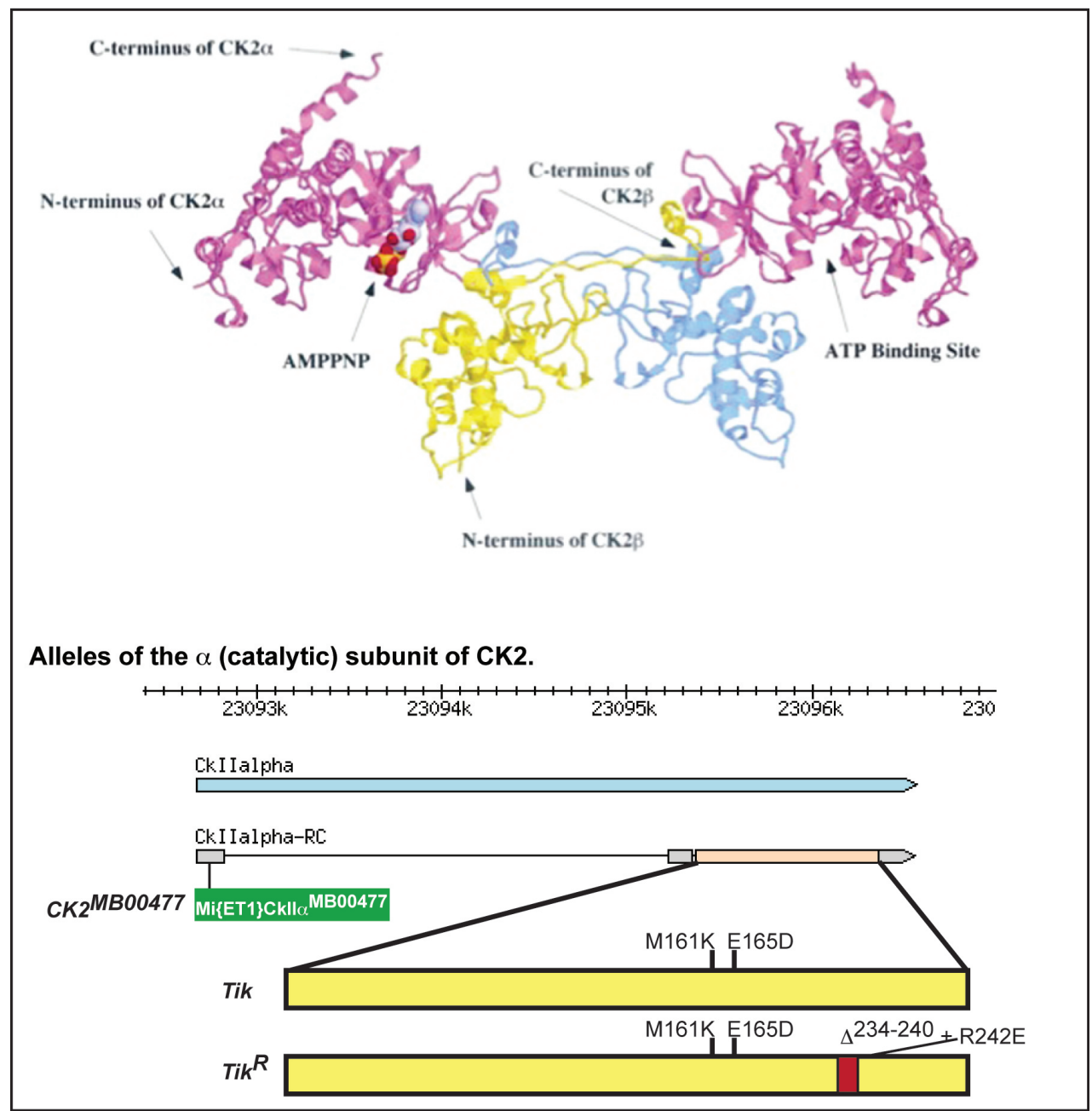

Fig. 8. Structure of the CK2 holoenzyme and the alleles of Drosophila CK2 $\alpha$ subunit are represented. Ribbon diagram of the human CK2 holoenzyme (described in Niefind et al., 2001). The two catalytic subunits (magenta), assemble on a CK2 $\beta$ dimer (yellow and blue). The alleles of Drosophila CK2 $\alpha, C K 2^{M B 00477}$, Tik and $T i k^{R}$ and their specific lesions are indicated. 


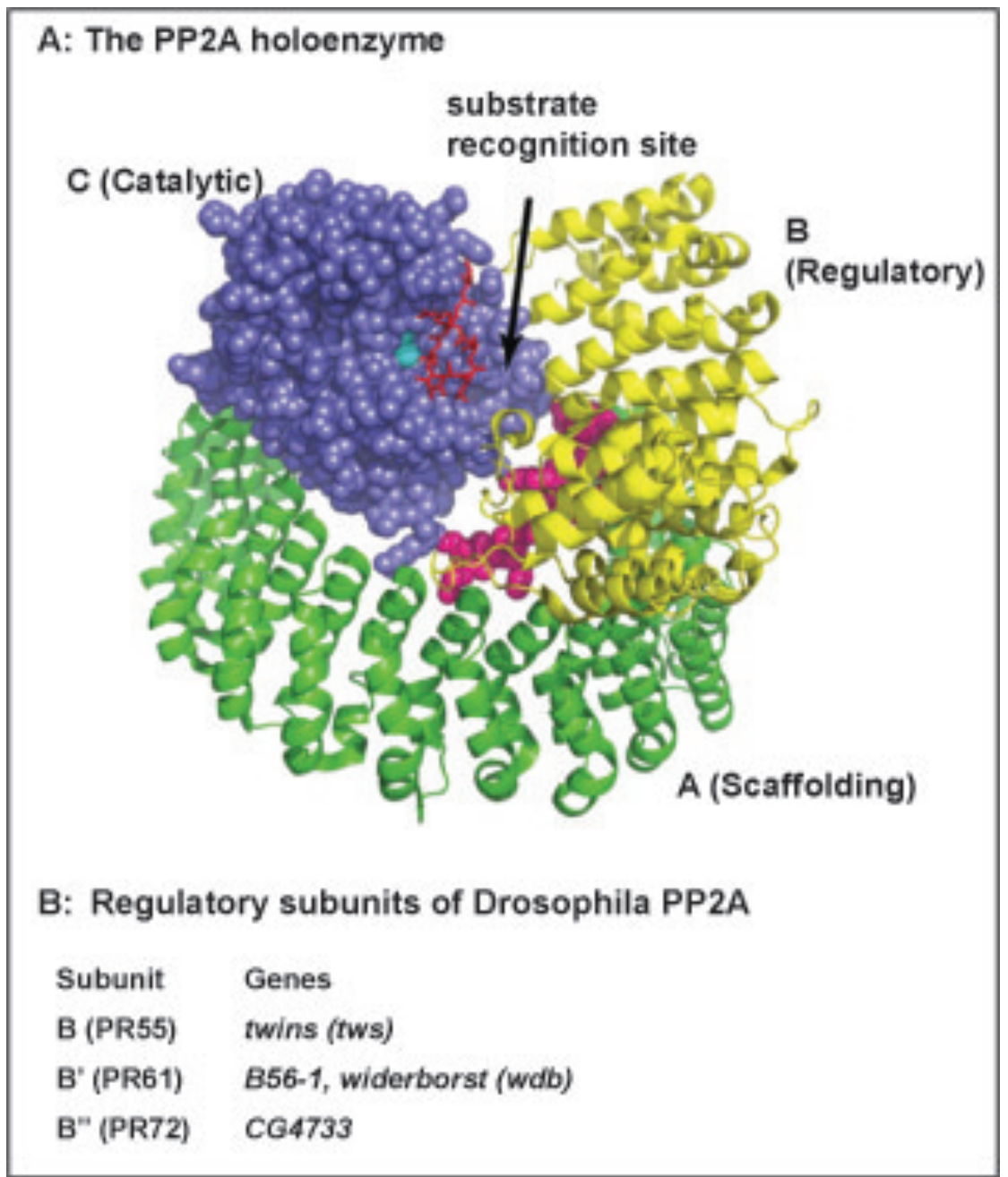

Fig. 9. The structure of PP2A holoenzyme and its regulatory subunits in Drosophila are represented. (A) The 3D structure of the PP2A holoenzyme containing the B56 $\gamma$ regulatory subunit (described in Mumby, 2007). The scaffold/A-subunit is depicted as green ribbon diagram. The catalytic subunit (Blue) and the regulatory subunit (yellow) are assembled on the A-subunit. (B) The names of the regulatory subunits in Drosophila and the corresponding genes are indicated (adapted from Sathyanarayanan et al., 2004). 


\section{Chapter 2}

Drosophila CK2 regulates lateral-inhibition during eye and bristle development. 


\section{Abstract:}

Lateral inhibition is critical for cell fate determination and involves the functions of $\operatorname{Notch}(N)$ and its effectors, the Enhancer of Split Complex, $E(s p l) C$ repressors. Although $\mathrm{E}(\mathrm{spl})$ proteins mediate the repressive effects of $N$ in diverse contexts, the role of phosphorylation was unclear. The studies we describe implicate a common role for the highly conserved Ser/Thr protein kinase CK2 during eye and bristle development. Compromising the functions of the catalytic $(\alpha)$ subunit of CK2 elicits a rough eye and defects in the interommatidial bristles (IOB's). These phenotypes are exacerbated by mutations in CK2 and suppressed by an increase in the dosage of this protein kinase. The appearance of the rough eye correlates, in time and space, to the specification and refinement of the 'founding' R8 photoreceptor. Consistent with this observation, compromising CK2 elicits supernumerary R8's at the posterior margin of the morphogenetic furrow (MF), a phenotype characteristic of loss of $E(s p l) C$ and impaired lateral inhibition. We also show that compromising CK2 elicits ectopic and split bristles. The former reflects the specification of excess bristle SOP's, while the latter suggests roles during asymmetric divisions that drive morphogenesis of this sensory organ. In addition, these phenotypes are exacerbated by mutations in CK2 or $E(s p l)$, indicating genetic interactions between these two loci. Given the centrality of $E(s p l)$ to the repressive effects of $N$, our studies suggest conserved roles for this protein kinase during lateral inhibition. Candidates for this regulation are the $E(s p l)$ repressors, the terminal effectors of this pathway. 


\section{Introduction:}

Animal development is predicated upon signaling pathways that drive cell fate determination with remarkable spatial and temporal precision. These pathways are highly conserved and reiteratively utilized in diverse developmental programs. An example is the Notch $(N)$ pathway whose activities regulate the development of sensory organs such as the eye and bristles, and during myogenesis, oogenesis, etc (reviewed in Artavanis-Tsakonas et al., 1999; Lai, 2004; Mumm and Kopan, 2000). This pathway consists of the receptor $N$, its ligands Delta $(D /)$ or Serrate (Ser), its mediator, the transcription factor encoded by Suppressor of Hairless $(S u(H))$, and its final effectors, the $E(s p l)$ repressors. The $E(s p l) C$ encodes seven basic-Helix-Loop-Helix (bHLH) proteins ( $\mathrm{M} \beta, \mathrm{M} \gamma, \mathrm{M} \delta, \mathrm{M} 3, \mathrm{M} 5, \mathrm{M} 7, \mathrm{M} 8)$ and the non-bHLH protein Groucho (Gro) (Delidakis and Artavanis-Tsakonas, 1991; Hartley et al., 1988; Klambt et al., 1989; Knust et al., 1992; Schweisguth and Posakony, 1992).

The roles of $N$ have been intensively studied during Drosophila neurogenesis, a process that leads to the stereotyped patterning of sensory organs, the bristles (macrochaetes) and the compound eye (reviewed in Baonza and Freeman, 2001; Bray, 1997; Campos-Ortega, 1997). The onset of neural development is characterized by the expression of the bHLH transcription factors (proneural activators) encoded by the achaete-scute complex (ASC) and atonal (ato) (Heitzler et al., 1996; Jarman et al., 1995; Jarman et al., 1994; Modolell and Campuzano, 1998; Skeath and Carroll, 1991). The expression of these activators occurs in groups of cells, the proneural clusters (PNC's), and this expression serves to maintain neural competency (reviewed in Calleja et al., 2002; Dambly-Chaudiere and Vervoort, 1998; Gibert and Simpson, 2003). However, from each PNC only a fixed number of cells go on to adopt the neural fate, while others are redirected away from this cell fate. This selection process is initiated when one cell of a PNC gains an advantage over its neighbors because it expresses the highest levels of ASC or Ato. This cell is destined to become the SOP, and it, in turn, inhibits other cells of the PNC from adopting the neural fate. This 
process, involving $N$, has been termed lateral inhibition (Lehmann et al., 1983; Simpson, 1990). Specifically, the cell destined to become the SOP expresses $D /$ which activates $N$ in other cells of the PNC (non-SOP's). Activated $\mathrm{N}$ is cleaved and its intracellular domain $\left(\mathrm{N}^{\mathrm{ICD}}\right.$ ) converts $\mathrm{Su}(\mathrm{H})$ from a repressor into an activator enabling transcription of $E(s p l) C($ Bailey and Posakony, 1995; Lecourtois and Schweisguth, 1995; Schrons et al., 1992). The E(spl) repressors (in a complex with Gro) then antagonize the proneurals activators (ASC, Ato) and prevent non-SOP cells from adopting the neural (SOP) fate; in the case of the bristle this is the epidermal fate. In the SOP, however, the high levels of ASC do not lead to transcription of $E(s p l) C$ due to the repressive effects of $\mathrm{Su}(\mathrm{H})$ in combination with $\mathrm{H}, \mathrm{CtBP}$, and Gro (Castro et al., 2005; Hinz et al., 1994; Koelzer and Klein, 2003; Kramatschek and Campos-Ortega, 1994; Singson et al., 1994). SOP selection also involves the functions of senseless (sens), a zincfinger transcription factor(Jafar-Nejad et al., 2003). As a result, lateral inhibition amplifies the neural (SOP) versus non-neural dichotomy in time and space. Consistent with this, overexpression of $A S C /$ ato or loss of $N$ signaling $(N, S u(H), E(s p l) C)$ skews the balance in favor of the SOP fate, and manifests as ectopic bristles (neural hyperplasia)(Campos-Ortega, 1998; Skeath and Carroll, 1991). In contrast, overexpression of $E(s p l)$ or loss of ASC/ato antagonizes the SOP fate, and manifests as neural hypoplasia (bristle suppression) (Giebel and Campos-Ortega, 1997; Nakao and Campos-Ortega, 1996; Tata and Hartley, 1995). The distinction between neural versus non-neural cell-fates is not the only function of $N$. For example, during macrochaete development, the SOP undergoes two asymmetric cell divisions to generate four cell types (socket, bristle, sheath and neuron), a process that also involves $N$ and $E(s p l)$. Interference with $N$ or $E(s p l)$ functions after SOP selection thus skews these fates, and elicits missing or split bristles (reviewed in Bray, 1997; Campos-Ortega, 1997 and see Section 3).

The stereotyped patterning of the eye also involves reiterated $N$ signaling. Retinal histogenesis initiates in the third larval instar when the first neurons, the R8's, are specified in 
the eye imaginal disc (Jarman et al., 1994). Because the specification of all other cell types depends on signals from the R8 cell, it is called the 'founding' photoreceptor. The specification of R8's occurs in a wave of differentiation, the morphogenetic furrow (MF), that sweeps across the eye disc (Wolff and Ready, 1991). During this process, the epithelium is transformed into a hexagonal array of ommatidia, each derived from a 'founding' R8. In this context, biphasic $N$ functions, separated by time and space, are required for R8 specification (Ligoxygakis et al., 1998). At the anterior margin of the MF, $N$ elicits ato expression in groups of cells akin to PNC's. This phase of $N$ is $E(s p l)$-independent (Baker et al., 1996). At the posterior margin of the MF, $N$ mediates expression of $E(s p l)$ which restrict Ato to a single cell from each cluster and this cell goes on to become the R8 photoreceptor. However, unlike the bristle, in the eye the remaining cells of this group are transiently blocked from the neural fate, thus allowing for their subsequent recruitment as secondary (R1-R7) photoreceptors. In addition to R8's, lateral inhibition by $\mathrm{E}(\mathrm{spl})$ is required during other steps of retinal patterning, and the specification of the IOB's whose developmental pathway shares many, but not all, of the genetic determinants that also specify the macrochaetes (Cadigan et al., 2002; Cadigan and Nusse, 1996; Cagan and Ready, 1989b; Frankfort et al., 2004).

In spite of an immense body of evidence on $N$ signaling, it had remained unknown if lateral inhibition by $\mathrm{E}(\mathrm{spl})$ proteins is regulated and, if so, by what mechanism(s). Our previous studies had implicated the Ser/Thr protein kinase, CK2, as a regulator of $\mathrm{E}(\mathrm{spl})$. Specifically, we had shown that a variant of E(spl)M8 that replaced Ser ${ }^{159}$, the CK2 phosphoacceptor, with the 'phosphomimetic' amino acid Asp (UAS-m8SD) generated an eyespecific dominant allele that mimicked (the reduced eye of) $E(s p l) D$, an allele of the $m 8$ gene (Karandikar et al., 2004). However, no allele-specific bristle defects were associated with these phosphorylation site variants. These results were enigmatic because the resolution of PNC's (SOP selection) during bristle patterning (lateral inhibition) employs mechanisms that are similar to those in the eye. Because a balance between proneurals and neurogenic 
proteins is crucial for proper neural patterning, we elected to directly address the role of this protein kinase in the eye and bristle.

We have compromised CK2 functions by two methods; UAS-RNAi against the catalytic subunit of this enzyme (CK2 $\alpha)$, or UAS-Tik encoding a dead catalytic subunit that behaves as a dominant-negative (CK2-DN, (Lin et al., 2002; Rasmussen et al., 2005)). We show that compromising CK2 by either approach elicits neural defects in the eye and bristle that are akin to those upon loss of $E(s p l)$. Furthermore, these phenotypes are exacerbated by mutations in $C K 2 \alpha$ or $E(s p l)$, providing evidence in favor of an interaction between these two loci. These neural defects reflect the specification of supernumerary 'founding' R8's and bristle SOP's, both characteristic of impaired lateral inhibition. These studies provide the first direct link between CK2 and neurogenesis and suggest a more general role for this enzyme in lateral inhibition.

\section{Results:}

\section{Role of CK2-mediated phosphorylation of E(spl)M8:}

We have previously reported studies implicating CK2 as regulator of E(spl)M8 in eye development (Karandikar et al., 2004). Our analysis of (UAS) constructs of $E(s p l) m 8$ that harbor alterations of the CK2 phosphoacceptor, Ser ${ }^{159}$, suggested a role in the eye (Fig. 1A, inset). Specifically, the 'phosphomimetic' variant, $m 8 S D$, elicited a severely reduced eye, reflecting attenuated R8's due to exacerbated antagonism of Ato. Similar eye defects are seen with $E(s p l) D$, an allele of $m 8$, that encodes a truncated protein known as $\mathrm{M}^{*}$ (Nagel et al., 1999; Nagel and Preiss, 1999), leading us to suggest that phosphorylation augments repression by $\mathrm{E}(\mathrm{spl}) \mathrm{M} 8$. In contrast, no allele-specific effects were found in the bristle upon (mis)expression of UAS-m8, -m8SA or $-m 8 S D$. This result was surprising because resolution of PNC's by lateral inhibition is similar in the eye and bristle (Fig. 1B, C), in that it requires $E(s p /) C$. How might one reconcile an eye-specific role for CK2? In the eye, recruitment of 
secondary R-cells (R1-R7), which is dependent on the 'founding' R8's, is completed in the third instar eye disc. In contrast, bristle SOP's are specified in the third instar wing disc, but their divisions and differentiation only occur in the pupal stage. We had speculated that while phosphorylation might allow for a rapid onset of M8 repressor activity, dephosphorylation following R8 refinement would avoid a protracted block of the neural fate, thus enabling recruitment of other R-cells. The possibility thus arose that this requirement for CK2 reflects intrinsic differences in the eye versus bristle, or is a limitation of analysis of ectopically (Gal4UAS) expressed $\mathrm{E}(\mathrm{spl})$ proteins. We considered the latter possibility more likely since proper neural patterning depends on a balance between $\mathrm{E}(\mathrm{spl})$ and ASC/Ato. To avoid skewing this balance, we compromised CK2 functions in the wild type, with the prediction that this should afford a precise and direct assessment of the roles of CK2 in the eye and the bristle. This approach was predicated on our observation that CK2 is expressed ubiquitously in the eye and the wing discs (data not shown).

\section{CK2-RNAi or CK2-DN perturb eye and bristle development:}

We have employed two approaches to compromise the catalytic subunit of CK2 (CK2 $\alpha$ ), i.e., RNA-interference (RNAi) and dominant-negative (DN) constructs. Our choice of these two approaches was based on the absence of conditional alleles or regulators of CK2 $\alpha$, and the difficulty of generating mitotic clones given that $C K 2 \alpha$ is centromere-linked, plays critical roles in the cell cycle and is required for cell-viability (Lin et al., 2002; Pinna, 2002b). We employed two constructs to target CK2 $\alpha$. These are, UAS-CK2 $\alpha-R N A i$ (referred to as CK2-RNAi) and UAS-Tik that was based on an allele of CK2 $\alpha$, called Timekeeper (Tik). Tik was identified in a screen for dominant modifiers of the circadian clock, and in a heterozygous condition displays no overt neural abnormalities apart from the clock defect (Lin et al., 2002). Tik harbors two substitutions, $\mathrm{M}^{161} \mathrm{~K}$ and $\mathrm{E}^{165} \mathrm{D}$. The former substitution resides in the ATP. binding pocket (Niefind et al., 2001), blocks nucleotide-binding and thus eliminates catalytic 
activity (Lin et al., 2002; Rasmussen et al., 2005). As a result, Tik homozygotes are lethal. The UAS-Tik construct was thus based on the expectation that ectopic Tik would incorporate into the endogenous CK2 holoenzyme, the heterotetramer of CK2 $\alpha$ and the regulatory subunit CK2 $\beta$ (Glover et al., 1983) and dominantly block functions, i.e., a UAS-Tik is a CK2-DN. We note that the holoenzyme is required for proper CK2 function in vivo (Jauch et al., 2002).

We elected to first use scaGal4 because this driver enables the simultaneous analysis of bristles and eyes. scaGal4 is active in neural precursors in the embryo, in PNC's in the imaginal discs, in SOP's and later in the lineage of the external sense organs, and in the MF of the eye disc (Nakao and Campos-Ortega, 1996). As expected, the Gal4 driver or the UASlines (CK2-RNAi or UAS-Tik) did not exhibit any eye or bristle abnormalities (Fig. 1D, E and data not shown). Surprisingly, expression of CK2-RNAi or UAS-Tik by scaGal4 did not perturb the eye (Fig. 1F, F', and data not shown), but led to subtle, yet reproducible, defects in patterning of the IOB's (Fig. 1F'). This was not due to non-functional CK2-RNAi or UAS-Tik constructs, because these flies exhibited defects in bristle patterning (Fig. $1 \mathrm{G}$ and see below). To test the possibility that the wild type eye reflected insufficient knockdown of CK2, we increased the dosage of scaGal4 and/or the CK2-RNAi insertions. We, however, found no ommatidial defects with 2xscaGal4 plus 2xCK2-RNAi (data not shown), conditions that led to more severe bristle defects such as ectopic, split and missing, than those associated with 1xscaGal4 plus 1xCK2-RNAi (Fig. 1G, H). Such a split bristle phenotype is not observed with homozygous scaGal4 or CK2-RNAi stocks.

\section{CK2-RNAi or CK2-DN leads to a rough eye phenotype:}

Given the dynamics of retinal patterning and the domain of scaGal4, we thought that a number of reasons might underlie the unperturbed eye. First, retinal (R8) patterning initiates in the MF (Treisman, 2004; Voas and Rebay, 2004; Wolff and Ready, 1991b). Second, expression of scaGal4 is restricted to cells at stage $2 / 3$ of the MF (Fig. 2A), where refinement of R8 'founders' occurs (reviewed in Hsiung and Moses, 2002). Third, because retinal 
histogenesis proceeds approximately one column of ommatidial founders (R8's) every two hours (Ready et al., 1976), scaGal4 mediated 'knockdown' might be delayed (hysteretic) relative to the time/space (the $\mathrm{MF}$ ) where $\mathrm{E}(\mathrm{spl})$ mediates $\mathrm{R} 8$ refinement. To uncover a role in the eye, it might thus be necessary to drive expression anterior to the sca domain (the MF). In the case of CK2-RNAi, this would permit turnover of endogenous CK2 to a rate-limiting level, whereas in the case of UAS-Tik, it would allow for accumulation of inactive CK2 $\alpha$ and 'poisoning' of the holoenzyme. We thus conducted analysis with Gal4 drivers whose expression patterns relative to the MF are well defined. These are, $h$ Gal4, eyGal4, $h t h G a l 4$, tshGal4, E(spl)Gal4, and gmrGal4 (Fig. 2A).

We find that eye defects are uncovered only when CK2-RNAi or UAS-Tik are expressed with eyGal4 (Fig. 2B). These include a rough eye and defective patterning of IOB's, both restricted to the posterior of the eye field (Fig. 2C, C', F). It is important to note that in the case of eyGal4+UAS-Tik, the perturbed eye was manifest at $29^{\circ} \mathrm{C}(\mathrm{Fig} .2 \mathrm{~F})$, but not at $24^{\circ} \mathrm{C}$ (data not shown), presumably reflecting increased expression. No such defects are associated with individual stocks, the corresponding controls (Figs. 1E, 2I), or when eyGal4+CK2-RNAi crosses are carried out at $18^{\circ} \mathrm{C}$ (see Fig. 3 inset). Because UAS-Tik did not appear as effective as CK2-RNAi, all further analysis was conducted with the latter construct. We next sought to determine if expression anterior to the MF with hth Gal4 and tshGal4 also elicits eye defects. However, expression of CK2-RNAi with both drivers was lethal at $18 / 24^{\circ} \mathrm{C}$ (Fig. 2B), presumably reflecting greater developmental expression (Fig. 2A and (Bessa et al., 2002)). Expression of CK2-RNAi in the MF with $E(s p l) G a l 4$ or posterior to it with gmrGal4 did not elicit any ommatidial/IOB defects (Fig. 2B), suggesting that the rough eye with eyGal4+CK2RNAi might not be due to a general 'sickness' of the eye disc.

\section{CK2-RNAi effects are exacerbated by Tik and suppressed by increased dosage of}

\section{$\mathrm{CK} 2 \alpha:$}

We next sought to confirm that the rough eye due to RNAi was target (CK2) specific. 
We thus conducted studies in a CK2 mutant background (Tik/+), with the expectation that exacerbation of the rough eye would provide evidence for specificity. We find that unlike the posterior restriction with $C K 2-R N A i$ alone, the rough eye encompasses virtually the entire eye field when eyGal4 drives CK2-RNAi in a Tik/+ background (compare Fig. 2C, D). In addition, these eyes displayed fused ommatidia (Fig. 2D') and patterning of the IOB's was more severly affected (compare Fig. 2C', D'). The ectopic IOB's are also characteristic of impaired lateral inhibition, and are in agreement with our studies on bristle patterning (see below). We do note that the eye field is slightly reduced in eyGal4/CK2-RNAi; Tik/+ animals. None of these phenotypes are associated with Tik/+ animals or other control combinations (Fig. 2G, I), suggesting that endogenous levels of CK2 are not rate-limiting (in the eye) and are specific effects of the Tik allele. We also tested whether the CK2-RNAi effects can be suppressed by the simultaneous expression of a UAS-CK $2 \alpha$ construct. This appears to be the case. A simultaneous increase in the dosage of $C K 2 \alpha$ reverses the effects of CK2-RNAi, wherein the hexagonal packing of the ommatidia and the patterning of the IOB's now appear virtually identical to the wild type (compare Fig. 2C', E'). By itself, overexpression of CK2 $\alpha$ does not lead to any eye defects (Fig. $2 \mathrm{H}$ ), confirming that CK2 $\alpha$ levels are not rate-limiting in this tissue. Together, these results suggest that the ommatidial and IOB defects of CK2-RNAi are target (CK2) specific.

Given the exacerbated effects of Tik, we next sought to determine whether this allele might provide a sensitized background, one where scaGal4+CK2-RNAi now elicits an eye defect. However, no eye defects were observed in scaGal4/+; CK2-RNAi/Tik flies, even though these displayed bristle abnormalities that were unique to this combination (Fig. 2I, and see below). It thus appears that for scaGal4 the absence of an eye defect reflects expression in the MF, and this is overcome by the more anterior expression with eyGal4. We also tested for heteroallelic interactions, but find no eye defects in Tik/E(spl)D flies (Fig. 2I). 


\section{The rough eye correlates to the onset of $\mathrm{R} 8$ patterning:}

While the rough eye of CK2-RNAi could reflect compromised lateral inhibition during R8 refinement, it could as well reflect indirect effects because this phenotype was uncovered when it was expressed anterior to the MF with eyGal4. This was a possibility since eyGal4 is active earlier in development, and we thus sought to assess the phenocritical period. For this, we have taken advantage of the observation that the eye defects of eyGal4+CK2-RNAi are temperature dependent, rough at $24^{\circ} \mathrm{C}$, but not at $18^{\circ} \mathrm{C}$ (Fig. $2 \mathrm{C}$ and inset in Fig. 3). Thus crosses were established at $18^{\circ} \mathrm{C}$, and vials were shifted to $24^{\circ} \mathrm{C}$ at four time periods after egg laying (AEL). Each temperature shift lasted for 24 hours, following which vials were returned to $18^{\circ} \mathrm{C}$ until eclosion. The four periods were chosen relative to the onset of $\mathrm{R} 8$ patterning (oRP) at 186 hours AEL (Kumar and Moses, 2001). A schematic of these temperature shifts is shown in Fig. $3(A-D)$, and the eye phenotypes are depicted in Fig. 3 ( $\left.A^{\prime}-D^{\prime}\right)$.

We find that temperature shifts at $150-174$ hours or $216-240$ hours do not perturb the eye (Fig. 3A', D'), suggesting that CK2-RNAi effects do not persist for long periods $(\geq 12$ hours), perhaps, due to continuous synthesis of new CK2. In contrast, a temperature shift from 192-216 hours, that overlaps the oRP (at 186 hours), elicited a rough eye (Fig. 3C'). However, temperature shifts at 168-192 hours did not elicit an eye defect, even though it overlapped oRP by 6 hours (Fig. 3B'). In this case, a likely reason for the wild type eye is that CK2-RNAi effects are hysteretic, and the refractory period represents the time required for turnover of CK2 protein to a rate-limiting level. Given the temporal dynamics of retinal patterning, i.e., one column of R8's every two hours (Wolff and Ready, 1991), we estimate that the hysteretic period for $C K 2-R N A i$ is roughly 4-6 hours, and this probably accounts for the inability of scaGal4, $E(s p l) G a l 4$ or $h$ Gal4 to elicit a perturbed eye (Fig. 2B). Examination of

Fig. 3C' supports this reasoning, because ommatidia display the normal hexagonal pattern for 2-3 rows at the posterior margin and the anterior half of the eye (Fig. 3C', asterisks), with roughening restricted to the posterior (dotted circle in Fig. 3C'). Thus the rough eye correlates 
to the onset of retinal (R8) patterning.

\section{Compromising CK2 elicits supernumerary R8's in the MF:}

We reasoned that the rough eye due to CK2-RNAi (Figs. 2C, 3C') might reflect altered patterning of R8's due to impaired lateral inhibition. In this regard, it is important to note that ato, the proneural for R8 cells, is expressed as a broad stripe at stage 1 of the MF (Fig. 2A,(White and Jarman, 2000)). This broad expression of ato is mediated by $N$ in an $E(s p l)$ independent manner (Ligoxygakis et al., 1998). By stage $2 / 3, N$ elicits $E(s p l)$ expression, which then antagonize Ato to mediate lateral inhibition and refinement of the R8's (see Fig. 2A). As a result, by stage 4, the 'founding' R8's are identifiable as precise phase-shifted Atopositive cells. Moreover, this phasing, which dictates the hexagonal patterning of the adult eye, is highly sensitive to mutations in $E(s p /) m 8$, i.e., the classical severe reduced eye of $N^{\text {spl }} / Y ; E(s p l) D /+$ flies (Nagel et al., 1999). Given that M8 is phosphorylated by CK2, we sought to assess whether the rough eye (of CK2-RNAi expressing flies) reflected defects at this critical step in retinal histogenesis. Crosses were established at $24^{\circ} \mathrm{C}$, and eye discs from late third instar larvae were immunostained for Ato. While discs derived from CK2-RNAi larvae (Fig. 4A) or eyGal4 alone (data not shown) exhibited a wild type pattern, discs derived from eyGal4+CK2-RNAi larvae displayed supernumerary R8's at the posterior margin of the MF (Fig. 4D). This phenotype is characteristic of impaired lateral inhibition during R8 refinement, and has been unambiguously demonstrated in clones lacking $E(s p l) C$ or $S u(H)$ (Ligoxygakis et al., 1998), or upon ectopic expression of a UAS-ato construct (White and Jarman, 2000). The extra R8's in the CK2-RNAi discs are not due to generalized hyperactivity of ato at stage 1 (akin to ato overexpression), because the R8 equivalence groups do not appear to be perturbed. Moreover, CK2-RNAi discs stained for the R8 marker, Sens (reviewed in Jafar-Nejad and Bellen, 2004), exhibit supernumerary Sens-positive cells (compare Fig. 4B, E), evidence that the extra Ato-positive cells do, in fact, differentiate into R8 photoreceptors. Consistent with this, double staining against Sens+ELAV displayed 
photoreceptor clusters containing two Sens-positive cells (data not shown).

\section{R8 patterning defects are not due to apoptosis:}

Given the role of CK2 in the cell cycle (Hanna et al., 1995; Pepperkok et al., 1994), we sought to confirm that the R8 patterning defects in CK2-RNAi discs were not a consequence of apoptosis due to a block in the cell cycle. Staining with Acridine Orange demonstrates that the pattern of apoptosis in discs expressing CK2-RNAi (Fig. 4F) appears similar to discs harboring (but not expressing) the CK2-RNAi construct (Fig. 4C), or those derived from eyGal4 alone (data not shown). Furthermore, disc size was unaffected (Fig. 4C, F). A low level of apoptosis is characteristic of eye discs at this developmental time point and has been well documented by others (Cagan and Ready, 1989a; Miller and Cagan, 1998; Wolff and Ready, 1991a). Thus the R8 patterning defects are not the consequence of general 'sickness' due to a defect in the cell cycle or apoptosis.

\section{Compromising CK2 leads to bristle abnormalities:}

As stated above, expression of CK2-RNAi or UAS-Tik by scaGal4 elicited bristle abnormalities. This result would suggest that the functions of this protein kinase might also apply to lateral inhibition during bristle SOP selection, a process mechanistically similar to that of the 'R8's in the eye, with respect to the nexus between $E(s p l)$ and ASC. We therefore sought to better define the nature of the bristle defects, with the expectation that commonalities in these two developmental contexts would make the roles of this enzyme more general than previously envisioned. We thus employed a number of Gal4 drivers that have been extensively used for bristle patterning. These are the scaGal4, G455.2 and $E(s p l) G a l 4$. We find that CK2-RNAi expression with all three drivers elicits ectopic bristles (Fig. 5), whereas the Gal4 drivers or CK2-RNAi or UAS-Tik insertions, by themselves, did not display any bristle defects (data not shown). Expression of CK2-RNAi by scaGal4 elicited ectopic bristles on the scutellum (Giebel and Campos-Ortega, 1997), and virtually identical results were observed upon expression of UAS-Tik (Fig. 5A, B). Flies expressing CK2-RNAi 
or UAS-Tik typically displayed $\sim 5.0 \pm 0.01$ bristles on the scutellum, a number significantly

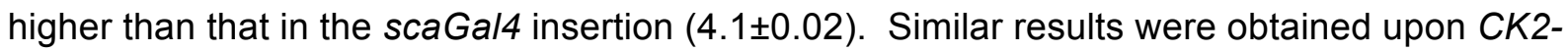
RNAi expression by the $\mathrm{G} 455.2$ insertion (Fig. $5 \mathrm{C}$ ), whose expression domain is restricted to the scutellum. In contrast, CK2-RNAi expression by $E(s p l) G a l 4$ led to the severest bristle defects which included the scutellum and the thorax (Fig. 5D).

We next employed an anti-Achaete (Ac) antibody to confirm that ectopic bristles reflect an increase in the SOP's in the wing disc. This study was based on observations that the PNC's give rise to macrochaetes that are characterized by unique positions, size and shapes of clusters, and time of specification (reviewed in Simpson et al., 1999). Consistent with the bristle defects, discs expressing CK2-RNAi display an increased number of Ac-positive cells in a position that corresponds to the scutellar clusters (Fig. 5E, F). Thus compromised CK2 favors the SOP fate, a phenotype that mimics loss of $E(s p l) C$. These results suggest that this enzyme also plays a role during lateral inhibition amongst PNC's in the bristle.

\section{Tik exacerbates the bristle defects of CK2-RNAi:}

We next sought to confirm that the ectopic bristles reflect compromised CK2 functions by testing for modulation of this phenotype by mutations in CK2. For this, we employed two alleles of CK2 $\alpha$, Tik and TikR (Lin et al., 2002). As mentioned above, Tik is a CK2-DN allele. In contrast, the revertant allele, TikR, deletes 7 amino acids and replaces $\operatorname{Arg}^{242}$ with Glu, in addition to the two original mutations seen in Tik. Thus, TikR is also likely catalytically inactive, but the deletion perturbs protein folding and prevents TikR from 'poisoning' endogenous CK2. We note that Tik/+ or TikR/+ flies do not display any bristle defects (Fig. $6 G)$.

We find unique bristle defects when scaGal4 drives CK2-RNAi in a Tik/+ background. These are, split bristles and branching of the shaft cell (Fig. 6A, A'). Neither phenotype is seen in the Gal4 or UAS stocks, or in other control combinations (Figs. 6G). While a split bristle is characteristic of impaired lateral inhibition, the 'branched' bristle might represent 
defects in shaft cell morphogenesis. Similar defects have been reported upon expression of UAS-m8 after SOP specification (Tata and Hartley, 1995) or in a 'gain-of-function' screen utilizing scaGal4 and the insertion line EP(3)0596 that interacts genetically with $H$ (AbdelilahSeyfried et al., 2000). While a mechanism for the 'branched' shaft in either instance remains to be defined, it is conceivable that in our case this reflects defects in cytoskeletal polarity due to a lower threshold of CK2 that is conferred by the (dominant) Tik allele. This interpretation would be consistent with just such a role (for CK2) in budding and fission yeast (Rethinaswamy et al., 1998; Snell and Nurse, 1994), and that altered cytoskeletal polarity elicits branched bristles in the fly ((Geng et al., 2000), and references within). In contrast, no split or 'branched' bristle defects are seen in scaGal4/+; CK2-RNAi/TikR flies (Fig. 6B). In this case, the ectopic bristles presumably reflect the baseline defects of scaGal4+CK2-RNAi (Fig. 5A). Allele-specific enhancement thus argues for a direct role for CK2 in the bristle lineage as well. One mechanism to account for the exacerbation (of bristle phenotypes) by Tik was that CK2 activity was decreased to an extent greater than that with CK2-RNAi alone. If this was the case, could this effect be mimicked by increasing the dosage of the CK2-RNAi construct. We find that expression of two copies of the CK2-RNAi construct also elicits 'branched' bristles (Fig. 6C, C'). Given that the 'branched' shaft is found by two independent routes, argues against the possibility that this is due to a secondary mutation in the Tik chromosome or is a mere artifact. On the contrary, it might reflect different thresholds of residual CK2 activity. To address the issue of thresholds of CK2 and the bristle phenotypes, we increased the dosage (2x each) of scaGal4 and CK2-RNAi, and find that the bristle phenotypes are more in line with defects in lateral inhibition. These are ectopic, split and missing macrochaetes (Fig. 6D). Occasionally split microchaetes were also observed (data not shown), as have also been shown upon ectopic expression of UAS-m5 (Tata and Hartley, 1995). SEM analysis of two closely placed bristles, one endogenous and one ectopic (Fig. 6E') indicates that both contain a socket cell, and their adjacent placement suggests impaired lateral inhibition during 
SOP selection. In contrast, the split bristle (Fig. $6 \mathrm{E})$ is reminiscent of those in $N^{s p l} / Y$; $E(s p l) D /+$ flies (Nagel and Preiss, 1999), and is likely to reflect altered sister cell fates, i.e., socket to shaft transformation (Fig. 1C). On the other hand, the missing bristle and its replacement by epidermal tissue (Fig. 6D) has been described previously for loss of function mutations in the $E(s p l) C$, and might have arisen due to a defect in the division of the SOP into plla and pllb sister-cell fates (Fig. 1C).

\section{CK2 genetically interacts with $E(s p I) D$ :}

We next sought to assess whether the bristle defects of CK2-RNAi are exacerbated by mutations in $E(s p l)$, as this would provide evidence for genetic interactions between these two loci. We elected to use the $E(s p l) D$ allele (Welshons, 1956), because this is the most restricted lesion in the $E(s p l) C$ and, most importantly, is one that mutates the $m 8$ gene, whose product is a CK2 target. We find that split bristles are elicited when scaGal4 drives CK2-RNAi in an $E(s p /) D /+$ background (Fig. $6 \mathrm{~F})$, while none of the relevant controls display such defects (Fig. 6G). Thus the split bristle is only observed upon an increased (2x) dosage of the scaGal4 and CK2-RNAi or when CK2-RNAi is expressed in an $E(s p l) D /+$ background (Fig. 6D, F). These results suggest that the effect of a double dose of CK2-RNAi is (pheno) mimicked by $E(s p l) D$, providing genetic evidence that supports the interaction of these two (CK2-M8) proteins.

\section{Discussion:}

\section{Lateral inhibition during eye and bristle development:}

Neurogenesis reflects the outcome of a complex balance between the activities of transcription factors that favor this cell fate (ASC/Ato) and those that oppose it $(\mathrm{E}(\mathrm{spl}))$. It is increasingly apparent that formation of the eye and bristle are predicated on a similar mechanistic framework, even though the proneurals that participate in these two developmental programs are distinct. For example, the PNC's in the eye (the R8 cell) require 
ato, while those in the bristle (macrochaetes and IOB's) require ASC (reviewed in Calleja et al., 2002; Gibert and Simpson, 2003; Hsiung and Moses, 2002). Nevertheless, one common feature of the resolution of PNC's in the eye and the bristle is the centrality of $E(s p /) C$, since loss of $E(s p l) C$ leads to exaggerated neurogenesis in both contexts (reviewed in CamposOrtega, 1997)). In the eye it leads to excess R8's, rough eyes, and duplicated IOB's, while in the bristles this manifests as ectopic, split and missing bristles. Extensive analyses have identified the genes involved with these developmental programs, the feedback loops that reinforce proneural expression in R8's/SOP's, and the role of $E(s p /) C$ for lateral inhibition (see introduction). In contrast, it has remained unclear how phosphorylation contributes to the dynamics of this process.

It has been thought that transcription of $E(s p l)$ was, by itself, necessary and sufficient for lateral inhibition. This model emerged from studies on bristle development, where ectopic $E(s p l)$ proteins extinguished the SOP's, whereas loss of $E(s p l)$ favored this cell fate (reviewed in Bray, 1997; Campos-Ortega, 1997). This model, we suggest, needs qualification because similar outcomes have not been recapitulated in the eye. In this context, loss of $E(s p l)$ demonstrably compromises lateral inhibition and elicits excess R8's (Ligoxygakis et al., 1998). However, ectopic expression of $E(s p l)$ members does not block the R8 fate, and consequently the eye displays the normal hexagonal packing of the ommatidia; the only defect is loss of the IOB's whose developmental program bears similarities to that of the macrochaetes (Karandikar et al., 2004). In contrast, R8 formation is blocked by the truncated M8* protein encoded by the $E(s p /) D$ allele, or by the CK2 phophomimetic variant M8SD (Fig. 1A). It is important to note that the eye defect of $E(s p l) D$ requires $N^{s p l}$, a recessive allele that attenuates ato, but not $E(s p l)$, expression (Baker et al., 1996). The inability of M8* to recruit Gro, which compromises repression, thus necessitates a sensitized background, one conferred by $N^{\text {spl }}$. Accordingly, M8SD (which binds Gro) elicits eye defects independent of $N^{\text {spl }}$ (Karandikar et al., 2004). Based on the observation that both M8* and M8SD display exacerbated and 
equivalent interactions with Ato, we proposed that CK2 phosphorylation switches M8 into an active repressor by uncovering the Orange domain, and it is this regulation that is bypassed by the $E(s p l) D$ mutation (Karandikar et al., 2004). Given that the Orange domain mediates binding to other proneurals as well, this regulation by CK2 should have been more general to lateral inhibition. The studies we report here suggest just such a role in the eye and the bristle.

\section{Roles for CK2 during lateral inhibition:}

Our studies support the notion that CK2 is a participant in lateral inhibition. We have shown that compromising CK2 by a number of independent routes, i.e., in wild type and backgrounds mutant for $C K 2$ and $E(s p l)$, elicits neural defects in the eye and bristle. These include rough eyes due to the specification of excess 'founding' R8 cells, and ectopic bristles (macrochaetes and IOB's) due to the specification of excess SOP's (Figs. 1, 2, 4, 5). These phenotypes are hallmarks of impaired lateral inhibition, and have been previously described for loss of function of the $E(s p l) C$ (reviewed in Campos-Ortega, 1997). We also provide evidence for genetic interactions between $C K 2$ and $E(s p l)$. While our studies provide multiple lines of evidence, the absence of suitable antibodies have precluded us from formally demonstrating that $\mathrm{E}(\mathrm{spl})$ repressors are, in fact, phosphorylated in cells undergoing lateral inhibition. Nevertheless, the congruence of our results utilizing CK2-RNAi or CK2-DN in conjunction with extant mutants and cell fate in imaginal discs, together, constitute a plausible argument supporting a role for this protein kinase.

Our studies also suggest secondary roles for CK2 in the bristle lineage. In contrast to R8 patterning, the roles of $N$ and $E(s p l)$ are different during bristle morphogenesis. In the case of the macrochaete or the IOB, $N$ and $E(s p l)$ are re-deployed following SOP selection (Fig. 7A). Specifically, the SOP gives rise to the pl neuroblast that undergoes two asymmetric divisions to generate four cell types characteristic of the sensillum; socket, shaft, sheath and neuron (Fig. 7A), and these divisions are dependent on $N$ - and $E(s p l)$-inhibitory 
signaling(Hartenstein and Posakony, 1990). Thus loss of $E(s p l)$ following SOP selection manifests as split bristles (aberrant division of the plla cell) or missing bristles (aberrant division of the pl cell). The split bristles we describe (Figs. 1,6) thus suggest a role for CK2 during the socket-to-shaft sister cell fate (Fig. 7A). In contrast, while the missing bristles (Fig. 6) suggest a role for CK2 during the plla-vs-pllb fates (Fig. 7A), this phenotype could result from loss of the SOP itself, a possibility if CK2 levels become rate limiting for cell division. Despite the fact that the timing of the asymmetric divisions of pl, plla and pllb are well known (see Fig. 1C), CK2-RNAi or CK2-DN are not suitable for dissecting the roles of CK2 at these later steps of bristle development. Conditional alleles of CK2, e.g., temperature-sensitives, will be necessary to better define its roles during specification of these sister-cell fates.

One major question that emerges from these studies is why is phosphorylation necessary, given that not all members of the $E(s p l) C$ are targets of $C K 2$. We think that evolutionary principles, the diversities and/or affinities of interactions between $\mathrm{E}(\mathrm{spl}) \mathrm{C}$ and ASC/ato, and their spatial expression patterns, perhaps, offer insights.

\section{A conserved role for CK2 in lateral inhibition:}

As stated above, of the seven $\mathrm{E}(\mathrm{spl})$ proteins, three (M8, M5, and $\mathrm{M} 7)$ are targeted by CK2, and these are also the most closely related (Ledent and Vervoort, 2001; Massari and Murre, 2000). Amongst all E(spl) members, two regions largely account for length heterogeneity and divergence (Delidakis and Artavanis-Tsakonas, 1991). These are sequences between HLH and Orange and those between Orange and WRPW, the CtD (Fig. 7B). However, within the CtD of M8 (and M5 and M7 as well) is a highly invariant sequence, the phosphorylation domain (P-domain) that harbors the CK2 site (Fig. 7B). Given the phylogenetic relationships of these species (Beverly and Wilson, 1984), it is noteworthy that over a period of $\sim 50$ million years the P-domain and the CK2 site have been remarkably conserved. For example, of all M8 homologs, only D. pseudoobscura, D. grimshawii and D. hydei harbor a Glu residue, in place of Asp, at the $n+3$ position of the CK2 phosphoacceptor. 
While we have not experimentally confirmed that these homologs are phosphorylated, the possibility is high because this change still conforms to the consensus (S/T-D/E-x-D/E) for recognition by CK2 (Kuenzel et al., 1987). We note that the virtually identical consensus site that is present in mammalian Hes6 is, in fact, targeted by CK2 in vivo (Gratton et al., 2003).

\section{CK2 phosphorylation and protein-protein interactions:}

The mechanisms by which $\mathrm{E}(\mathrm{spl})$ proteins mediate repression have been intensely studied. In essence, E(spl) proteins repress ASC/Ato. Repression was initially thought to involve binding to a DNA sequence, the N-box (Jennings et al., 1999; Jimenez and IshHorowicz, 1997). This, however, is not the case, because $\mathrm{E}(\mathrm{spl})$ proteins neutralized for DNAbinding still function as potent repressors (Giebel and Campos-Ortega, 1997). Furthermore, no $\mathrm{N}$-box has been found in the regulatory region of ato (Sun et al., 1998), while that in sc is dispensable for repression in non-SOP's (Culi and Modolell, 1998). It is now thought that direct (protein-protein) interactions between $\mathrm{E}(\mathrm{spl})$ and proneurals are more critical for repression, the protein-tether model (Giagtzoglou et al., 2003). In this model, repression by $E(s p l)$ occurs via direct interactions with enhancer bound proneurals, rather than by activator sequestration. This model is consistent with direct interactions between $\mathrm{E}(\mathrm{spl})$ and ASC/Ato proteins (Alifragis et al., 1997; Gigliani et al., 1996; Nagel and Preiss, 1999). It was, in fact, the analyses of various binary combinations that were the first to suggest that these interactions are regulated and non-redundant, two aspects that appear relevant to our findings.

Analysis of $\mathrm{M} 8$ and its $E(s p l) D$ encoded variant, $M 8^{*}$ provided the first hint that these antagonistic interactions are regulated. For example, Nagel and Preiss (1999) reported that, in addition to Ato (see above), M8* interacts with a much higher affinity with Ac, Sc, and Ase (they did not test L'sc). We have described a similar case for M8SD, which interacts with Ato or L'sc with affinities significantly higher than M8 or its non-phosphorylatable variant M8SA ((Karandikar et al., 2004), and our unpublished data). It is noteworthy that phosphorylation of 
mammalian Hes6 by CK2 (Fig. 7A) is also a pre-requisite for its interactions with Hes1(Gratton et al., 2003). Thus CK2 phosphorylation influences antagonistic interactions between the $\mathrm{E}(\mathrm{spl})$ and the ASC/Ato. Because these studies have employed two hybrid, instead of direct protein, approaches the possibility that these are kinetic effects remains open. This interpretation is consistent with the observations that a $2 x$ dosage of a UAS- $m \delta$ construct interferes with Ato and blocks eye development in the wild type(Ligoxygakis et al., 1998), whereas that of $m 7, m 5$ or $m 8$ requires $N^{s p l}$. Together, these findings argue that $\mathrm{E}(\mathrm{spl})$-ASC interactions are of variable strengths and are isoform-specific (Alifragis et al., 1997). Given that only a subset of $E(s p l)$ and $A S C$ members are expressed in the eye and wing disc (Cooper et al., 2000; Cubas et al., 1991; Koelzer and Klein, 2003; Lecourtois and Schweisguth, 1995; Skeath and Carroll, 1991), the possibility thus arises that distinct domains of ASC define sub-regions of the proneural field (reviewed in Gibert and Simpson, 2003). In this context, $E(s p l)$ members might have been selected based on their affinities and/or specificities for these proneural factors. Thus the type of $E(s p l)$ repressors that are deployed might reflect the combinations and levels of proneurals, with CK2 playing an integrative role. The currently available techniques preclude a distinction between these possibilities.

It is presently unclear if/how CK2 activity is modulated during neurogenesis. Expression of this enzyme appears to be constitutive in the eye and wing disc (Karandikar et al., 2004, and our unpublished data). Holoenzyme formation, proposed to be a dynamic process in vivo (Filhol et al., 2003), represents an attractive regulatory mechanism, given that CK2 $\beta$ modulates substrate recognition and that the fly $C K 2 \beta$ gene encodes for non-redundant isoforms of this regulatory subunit (Bidwai et al., 1993; Jauch et al., 2006). Alternatively, CK2 might be regulated by assembly into multiprotein complexes and/or via interactions with protein phosphatases (Giot et al., 2003; Heriche et al., 1997). Such a coordinated function has been described for regulation of Period, the central component of the circadian clock, by CK2 and the phosphatase PP2A (Lin et al., 2002; Sathyanarayanan et al., 2004). Future 
studies aimed at the identification of protein phosphatase(s) that counteract the phosphorylation of $\mathrm{E}(\mathrm{spl}) \mathrm{m} 8 / 5 / 7$ by $\mathrm{CK} 2$, or multiprotein complexes containing $\mathrm{E}(\mathrm{spl})$ and/or CK2 will be required to better define the regulatory dynamics of this process during eye and bristle development.

\section{Experimental Procedures}

\section{Fly stocks:}

Flies were raised at $24^{\circ} \mathrm{C}$ on standard Yeast-Glucose medium or at $18^{\circ} \mathrm{C}$ when indicated. The Gal4 drivers were generously provided by other researchers or obtained from the Bloomington Stock Center (denoted by the prefix B). These drivers are; G455.2, scaGal4, hGal4 (B1734), gmrGal4 (B1104), hthGal4 and tsh-Gal4, eyGal4 (ey3.5-Gal4(II), B8220), and $E(s p l) G a l 4$ (B8225). Two different insertions of UAS-RNAi flies (gift of Rob Jackson), or UASTik were used in these studies. The Tik and TikR alleles have been described, as is the $E(s p l) D$ stock (B2447).

Fly crosses and phenotypes:

All crosses were performed at $24^{\circ} \mathrm{C}$, unless indicated otherwise. Fly heads were passed through a graded alcohol series for 24 hours each (25-50-75-absolute). Finally, heads were passed through Hexamethyldisalizane, and mounted on EM stubs using carbon tape (Ted Pella). Fly heads were dried for 24 hours, sputter coated with gold, and examined with a JEOL-6400 scanning electron microscope at an accelerating voltage of $20 \mathrm{kV}$. Images were acquired, processed with Adobe Photoshop and collated in Adobe Illustrator. For bristle phenotypes, newly eclosed adults were photographed using a Nikon digital camera attached to a Leica MZ16 stereomicroscope. Alternatively, nota were processed for SEM analysis as described for the eye. For quantitative analysis of the bristle phenotypes, crosses were established in triplicates, and newly eclosed adults were scored for bristle artifacts. In every case between 100-250 flies were scored. 


\section{Immunostaining and cell death analysis:}

Imaginal discs were isolated from late third instar larvae and processed as described with modifications. Discs were fixed in $4 \%$ paraformaldehyde in $1 x$ phosphate buffered saline (PBS) for 15 minutes, and washed three times with PBS containing $0.1 \%$ Triton X-100 (PBSTX). The discs were incubated for 12 hours at $4^{\circ} \mathrm{C}$ in PBS-TX containing $5 \%$ normal goat serum and then immunostained. The following antibodies were used in this study: rabbit antiAto (gift of Yuh Nung Jan) at a dilution of 1:5000 and guinea pig anti-Sens (gift of Hugo Bellen) at a dilution of 1:500. Secondary antibodies (Molecular Probes) were goat-anti rabbitIgG coupled to Alexa Fluor 594 (1:800) and donkey anti-guinea pig-IgG coupled to Alexa Flour 546 (1:500). Wing discs from late third instar larvae were processed similar to the eye discs. PNC's were identified using a monoclonal anti-Achaete at a dilution of 1:20 followed by goatanti mouse-lgG coupled to Alexa Fluor 594 (1:800). The anti-Achaete antibody developed by Jim Skeath was obtained from the Developmental Studies Hybridoma Bank developed under the auspices of the NICHD and maintained by The University of lowa, Department of Biological Sciences, lowa City, IA 52242. Discs were mounted in Vectashield. For apoptosis assays, eye discs were isolated from late third instar crawling larvae in Drosophila Ringers, and stained with $1 \mathrm{mM}$ Acridine Orange (Sigma) for 5 minutes. Discs were rinsed once in Ringers, and imaged without any further processing.

\section{Confocal microscopy:}

An Olympus FluoView (FV1000) was used for confocal imaging. Images in Figs. 4 and 7 were generated from scans acquired every $1 \mu \mathrm{m}$ along the apicobasal axis of the discs. The scanning was limited along the Z-axis to acquire full spectral output of the fluorophores. Fluorophores were excited using appropriate excitation wavelengths. Individual Z-axis images were acquired, compressed as a Z-stack, and exported as TIFF files that were processed in Adobe Photoshop and collated in Adobe Illustrator. 


\section{Acknowledgments:}

We are grateful to Yuh Nung Jan for anti-Atonal antibodies, Hugo Bellen for antiSenseless antibodies, and Claiborne Glover for anti-CK2 antibodies. We thank Claude Desplan, Rob Jackson, Anette Preiss, Utpal Banerjee and the Bloomington Stock Center for fly stocks. We thank Diane Schwegler-Berry (NIOSH, CDC) for assistance with SEM analysis. 
A: Phenotypes with CK2-specific variants of E(spl)M8:

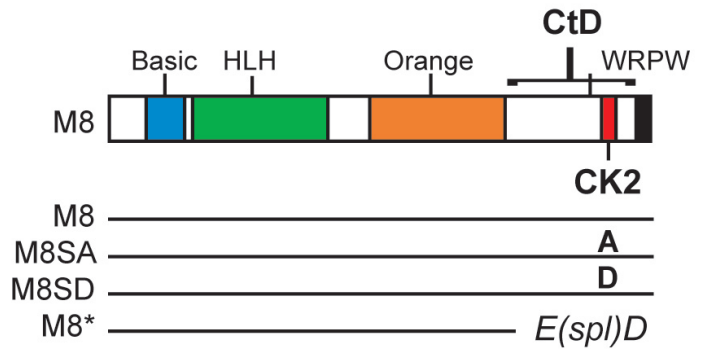

\begin{tabular}{|ll|}
\hline \multicolumn{2}{|l|}{ Eye Phenotypes } \\
UAS-m8 & Wild Type \\
UAS-m8SD & Reduced $^{1}$ \\
UAS-m8SA & Rough $^{2}$ (but not reduced) \\
UAS-m8* & Wild type $^{3}$ \\
UAS- $m 8^{*}$ & Reduced $^{4}$ (in NSpI/M) \\
\hline
\end{tabular}
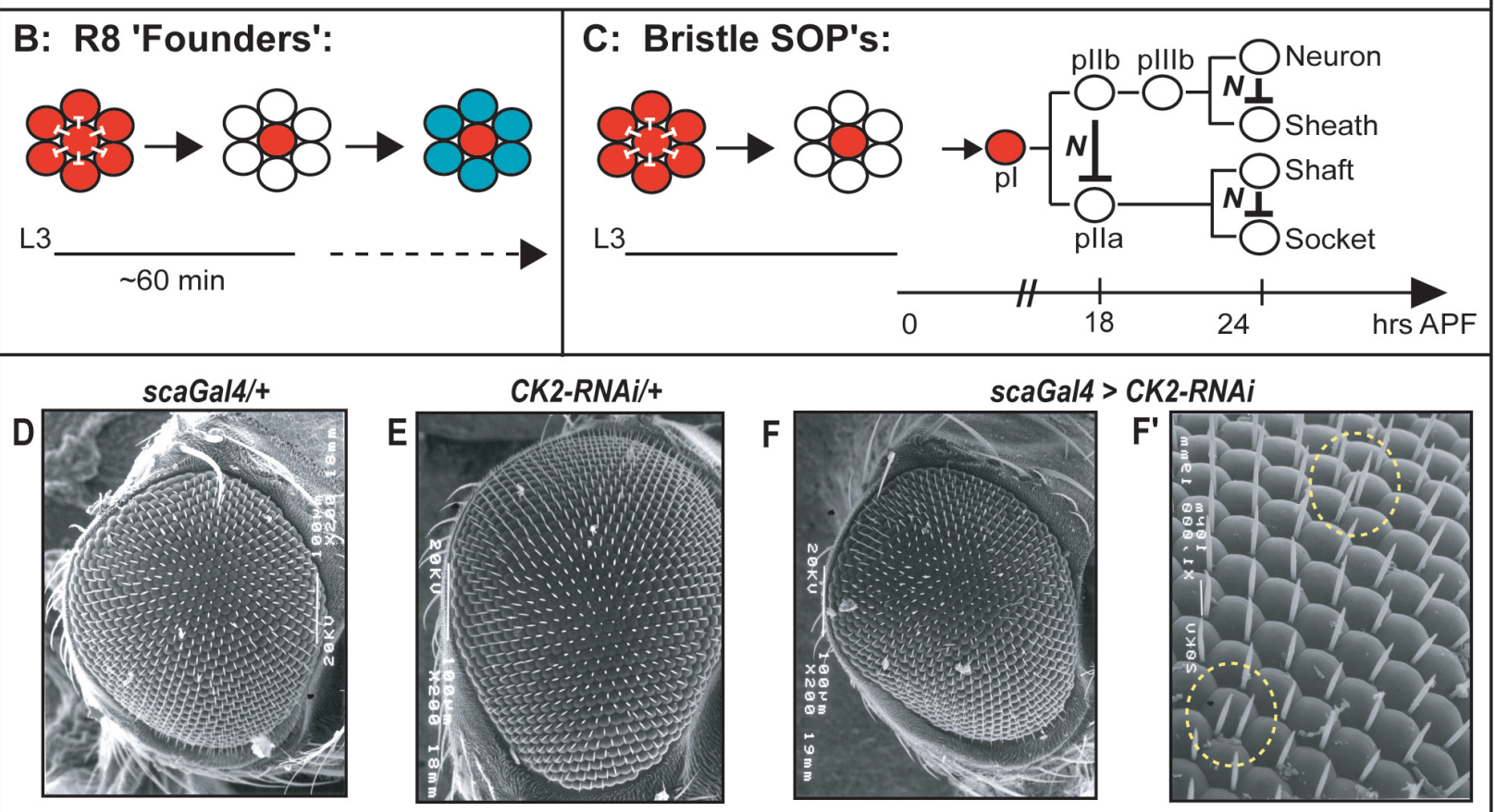

G

$$
\begin{array}{ll}
\frac{\text { scaGal4 }}{+} ; \frac{\text { UAS-CK2-RNAi }}{+} & \text { Ectopic } \\
\frac{\text { scaGal4 }}{\text { scaGal4 } ;} \frac{\text { UAS-CK2-RNAi }}{\text { UAS-CK2-RNAi }} & \begin{array}{l}
\text { Ectopic } \\
\text { Split } \\
\text { Missing }
\end{array}
\end{array}
$$

$2 X s c a G a l 4>2 X C K 2-R N A i$

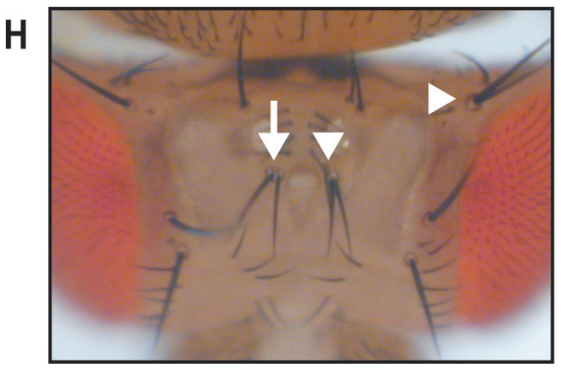


Fig. 1. (A) Summary of eye phenotypes elicited by (mis)expression of $E(s p l) m 8$ and its CK2 specific variants. M8SA and M8SD are Ala and Asp substitutions of $\operatorname{Ser}^{159}$, the CK2 phosphorylation site (SDCD), the deletion in $E(s p /) D\left(M 8^{*}\right)$ is indicated. Eye phenotypes have been described previously by others and us. Superscripts 1-4 indicate conditions leading to ommatidial defects. ${ }^{1}$ Severely reduced eye in $s c a G a / 4>U A S-m 8 S D$ flies. ${ }^{2}$ Rough eye in 109-68>UAS-m8SA flies, necessary to overcome lethality of scaGal4>UAS-m8SA. ${ }^{3}$ The wild type eye in $h^{H 10} \mathrm{Gal} / \mathrm{UAS}-m 8^{*} .{ }^{4}$ Severely reduced eye of $h^{H 10} \mathrm{Gal} / 4 \cup A S-m 8^{*}$ in a $N^{s p l} / Y$ background is similar to that in $N^{s p l} / Y ; E(s p l) D /+$. (B-C) Schematic of refinement of the R8 'founders' and bristle SOP's. Phases during which lateral inhibition by $\mathrm{N}$ mediates cell fate specification are indicated. The glial cell is omitted in panel $\mathrm{C}$ for simplicity. The effect of a UAS-CK2a-RNAi construct (abbreviated as CK2-RNAi) was assessed following expression with scaGal4. The genotypes are indicated and magnifications are $200 x$ for panels $D-F$ (posterior is towards the left), $F^{\prime}$ is a $1000 x$ magnification of a region of panel $F$. (G) Bristle abnormalities of flies with one or two copies of the scaGal4 and UAS-CK2-RNAi insertions. $(\mathrm{H})$ Bristle defects in $2 x s c a G a / 4>2 x C K 2-R N A i$ flies; ectopic bristle (arrow) and split bristles (arrowheads). 


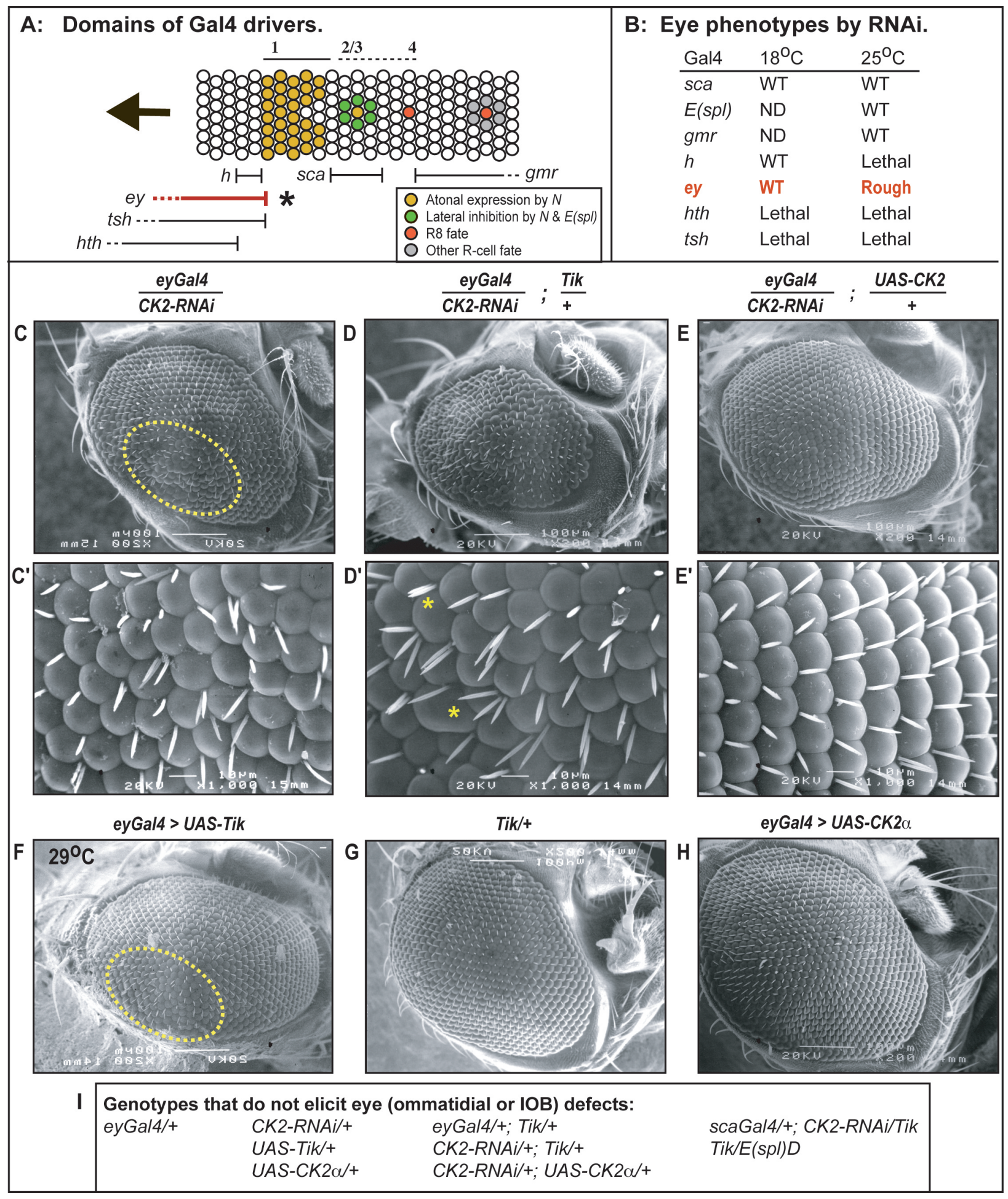


Fig. 2. Compromising CK2 elicits eye defects. (A) Expression domains of Gal4 drivers are shown relative to the MF of the eye disc. Cells at stage 1 are at the anterior margin of the MF (solid line) while those at stage 4 are at its posterior margin in accordance to the description of Frankfort and Mardon (2002). The arrow denotes MF progression. Inset described color scheme. The expression of Gal4 drivers relative to the MF are denoted. Dotted lines indicate expression earlier in development (ey, hth, tsh) or posterior to the MF (gmr). (B) Summary of eye phenotypes (WT denotes wild type and ND denotes not determined). Eye phenotypes were determined by SEM of the indicated genotypes at $24^{\circ} \mathrm{C}$ $(\mathrm{C}, \mathrm{D}, \mathrm{E}, \mathrm{G}, \mathrm{H})$ or at $29^{\circ} \mathrm{C}(\mathrm{F})$. Asterisks in panel D' denote fused ommatidia. Magnification is 200x (C, $D, E, F, G, H$ ) or 1000x (C', D', and E'). Dotted circles in $C$ and $F$ denote region of roughening. 


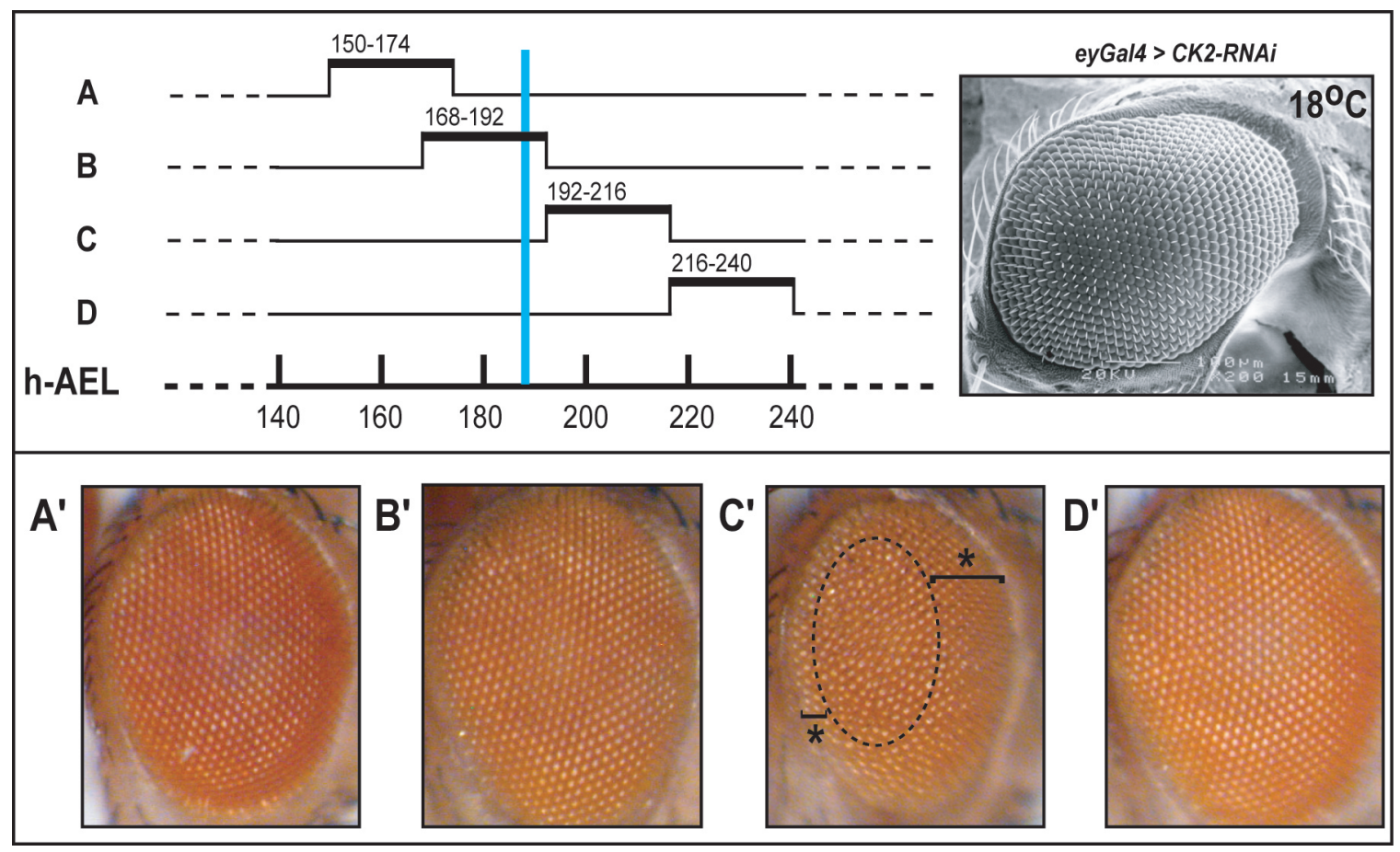

Fig. 3. The rough eye due to CK2-RNAi correlates to the onset of retinal patterning. Crosses of eyGal4 and CK2-RNAi were established at $18^{\circ} \mathrm{C}$. The vials were shifted the indicated times to $24^{\circ} \mathrm{C}$, and following a $24 \mathrm{~h}$ period were returned to $18^{\circ} \mathrm{C}$. Adult eye phenotypes were documented by photomicroscopy. The temperature shifts A-D are shown relative to the onset of retinal (R8) patterning (oRP, vertical blue line), and their corresponding effects on the eye are shown in panels A'-D'. Posterior is to the left. In panel C', asterisks denote normal phasing of ommatidia, and dotted circle denotes aberrant ommatidial phasing. 


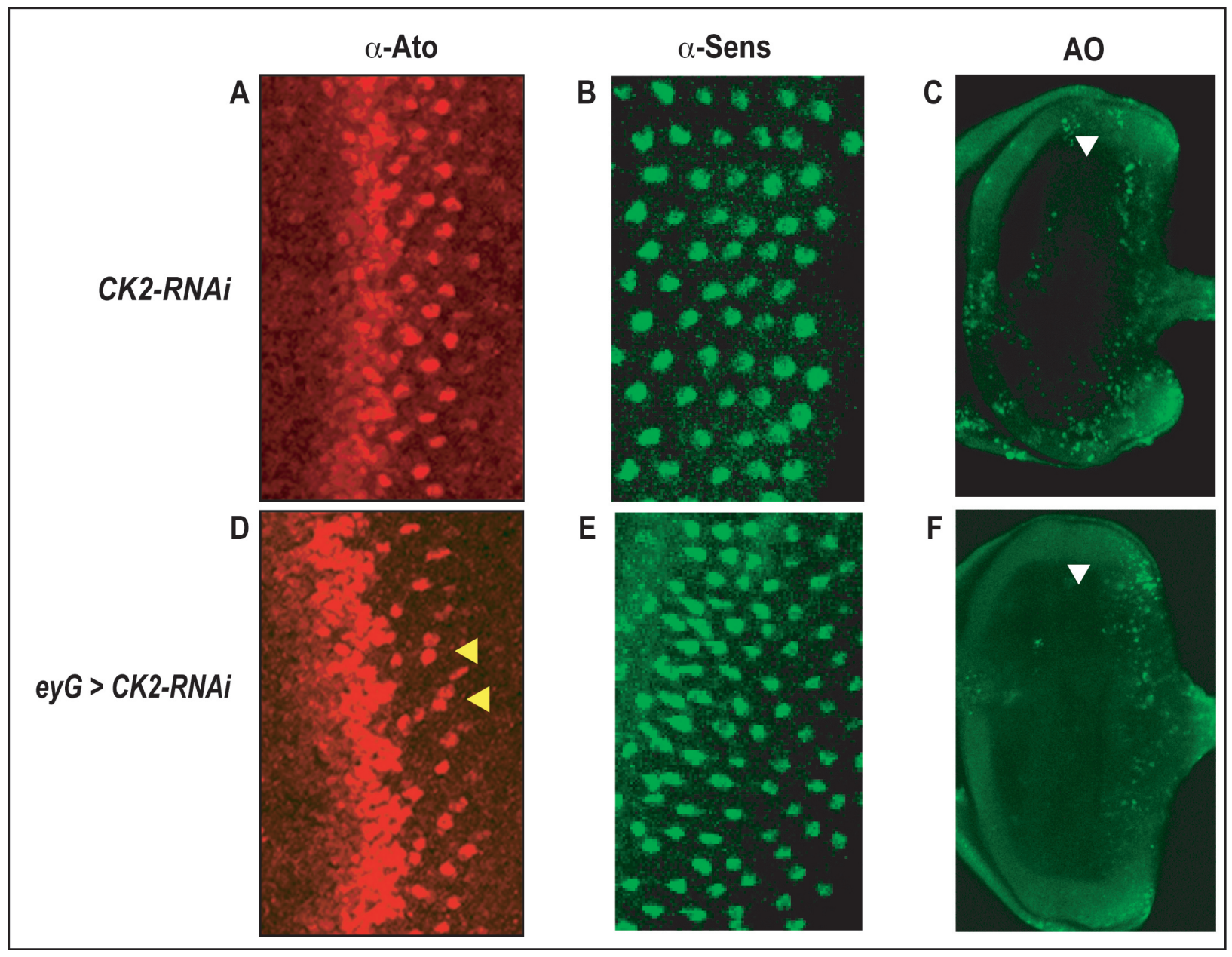

Fig. 4. Compromising CK2 elicits supernumerary R8's. Eye discs of the indicated genotypes were isolated and processed as described. A-C are discs harboring, but not expressing, the CK2-RNAi construct, whereas D-F are discs where CK2-RNAi was expressed by eyGal4 (eyG). Immunostainings were conducted using a-Atonal $(A, D)$, a-Senseless $(B, E)$, and Acridine Orange $(C, F)$. Posterior is to the right in each panel. Supernumerary R8's posterior to the MF are indicated by arrowheads in panel D. Only the relevant area of the eye disc is shown for a-Ato and a-Sens immunostainings. Arrowheads in $\mathrm{C}$ and $\mathrm{F}$ denote the MF. 


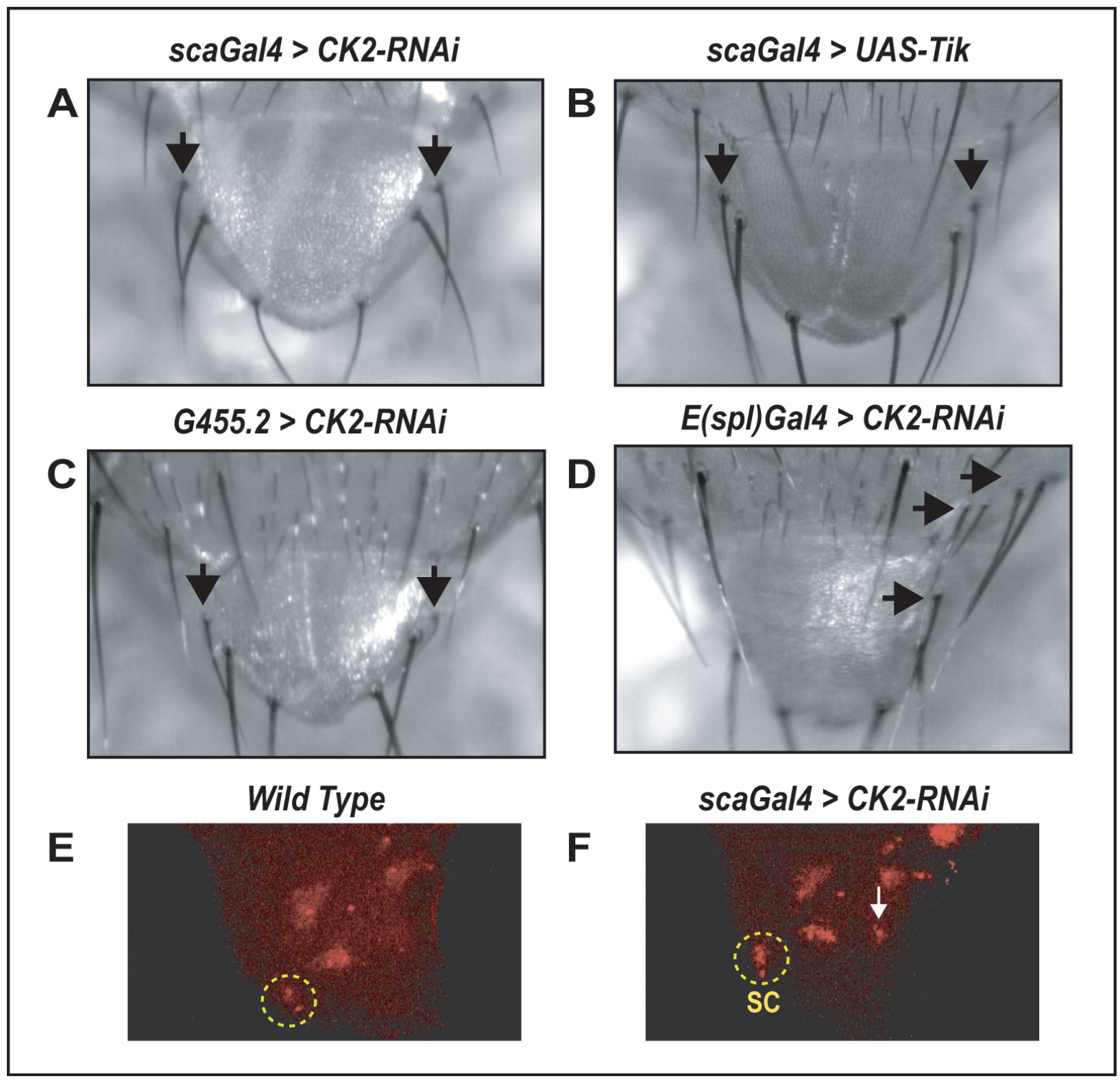

Fig. 5. Compromising CK2 elicits bristle patterning defects. The effect on bristle development was assessed following expression of the UAS-CK2-RNAi or a CK2-DN (UAS-Tik) constructs with scaGal4 (A, B), G455.2 @ , E(spl)Gal4 (D). Arrows denote ectopic bristles. Wild type and CK2-RNAi expressing wing discs were immunostained using a-Achaete $(E, F)$. Dotted circles denotes the scutellar PNC's, and the arrow in $\mathrm{F}$ denotes ectopic Ac-positive cells. 


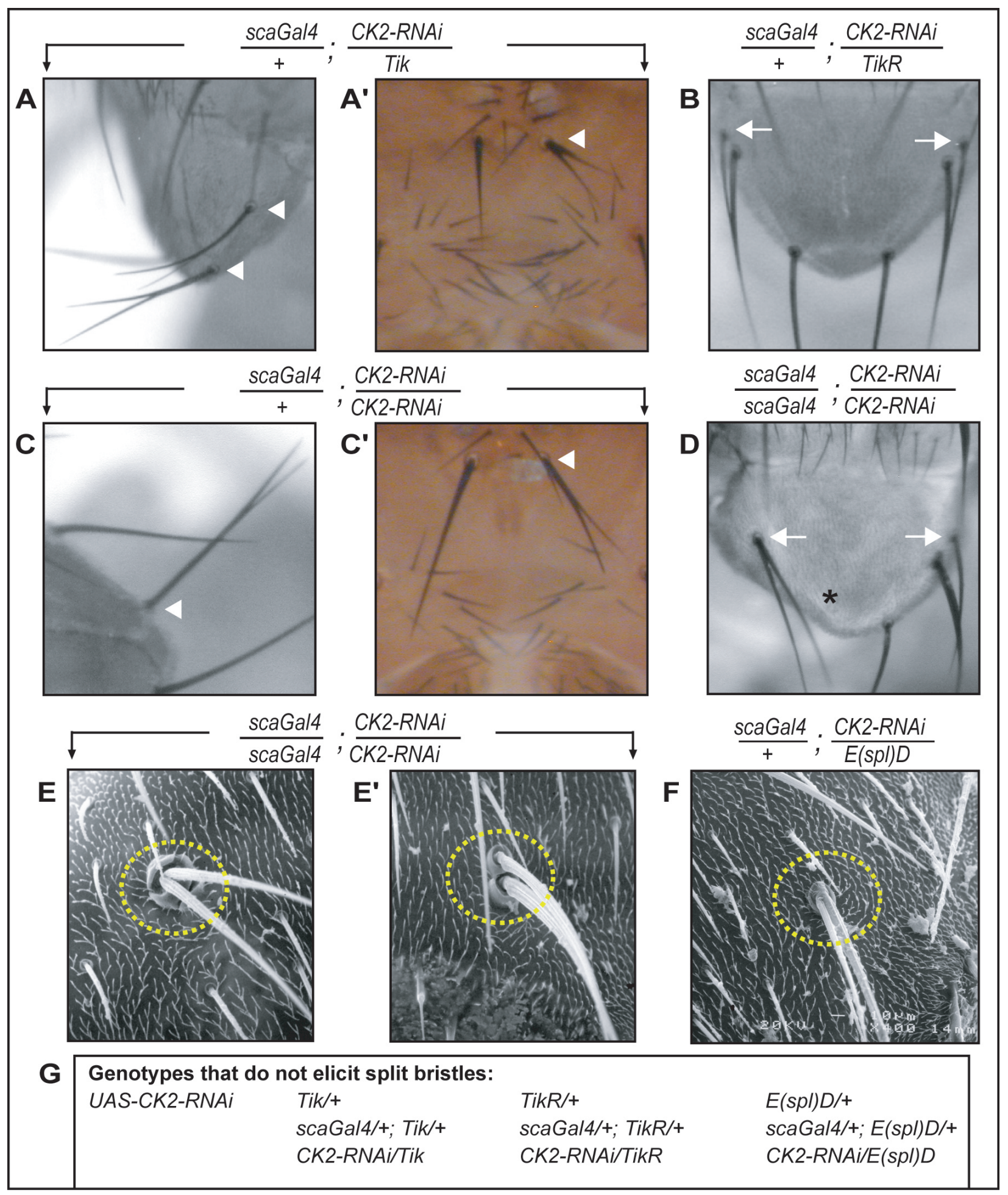


Fig. 6. Mutations in $C K 2$ or $E(s p l)$ modulate bristle defects of CK2 compromised flies. Bristle development was assessed following expression of a CK2-RNAi construct by scaGal4. Genotypes are indicated. Panels A-D are light micrographs of the scutellum (A, B, C, D) or the head ( $\left.A^{\prime}, C^{\prime}\right)$. Panels $\mathrm{E}, \mathrm{E}$ ' and $\mathrm{F}$ are SEM's. Arrows denote ectopic/split bristles, arrowheads denote 'branched' bristles, and asterisk denotes missing bristle and its replacement by epidermal tissue. 


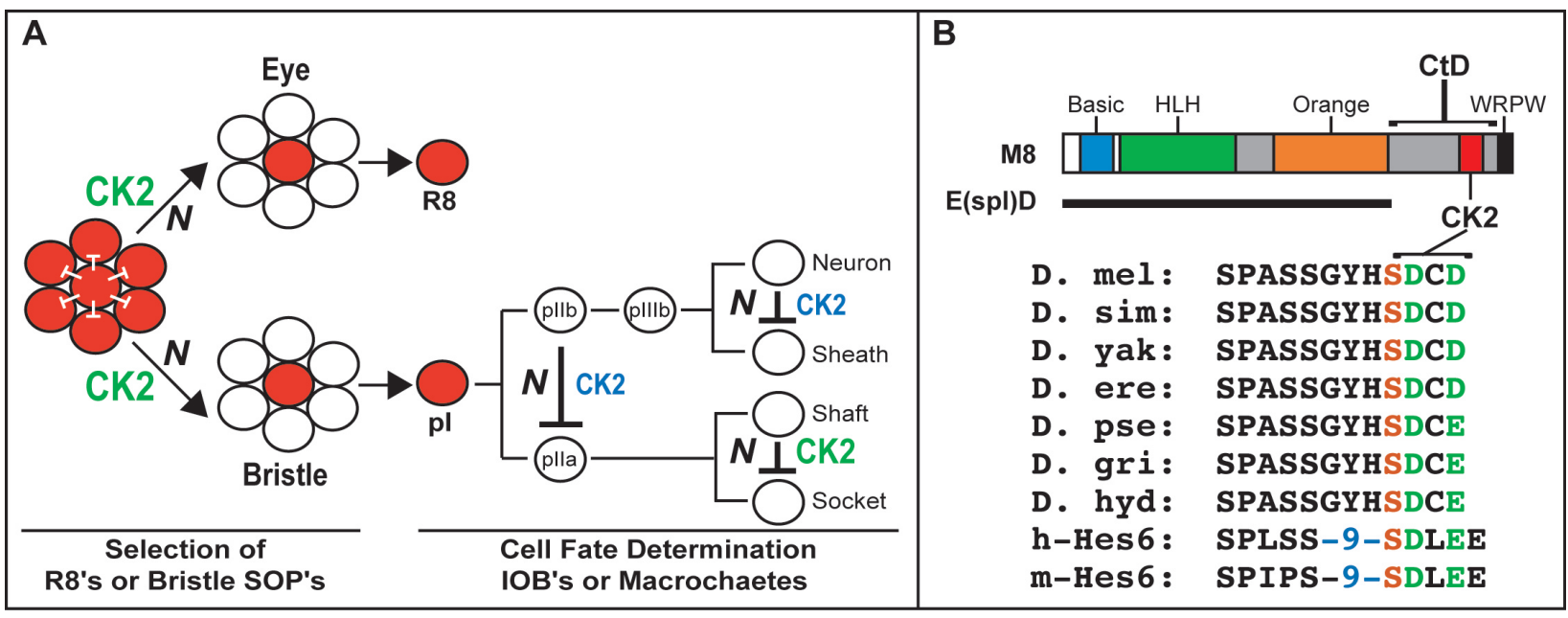

Fig. 7. Roles for CK2 during lateral inhibition. (A) Schematic of selection of R8's/SOP's from proneural clusters and SOP lineages in the bristle. The R8/SOP, which express high levels of the proneural proteins Ato or Ac, elicit inhibitory signaling via activation of Notch (white lines). Cells receiving this signal express $E(s p l)$ repressors, which redirect their cell fate away from the R8/SOP fate (shown in white). In the bristle lineage, the SOP gives rise to the pl neuroblast which divides asymmetrically to give rise to the second order precursors plla and pllb. Division of the plla gives rise to the socket and shaft cells, while pllb divides to generate a glial cell (not shown) and a third order precursor plllb. Finally, plllb divides to give rise to the neuron and sheath cells. These asymmetric divisions are also mediated through inhibitory Notch signaling (black lines) and involve the activities of $\mathrm{E}(\mathrm{spl})$ repressors. Steps requiring CK2 functions inferred from genetic analysis are shown in green letters, while blue letters denote steps where CK2 functions are predicted. (B) Schematic of the domains in $\mathrm{E}(\mathrm{spl}) \mathrm{M} 8$. The grey boxes denote linker regions, and the red box denotes the Phosphorylation-domain (P-domain) that is conserved amongst M5/7/8 and harbors the consensus site for phosphorylation by CK2. Sequences are; mel, melanogaster; sim, simulans; yak, yakuba; ere, erecta; pse, pseudoobscura; gri, grimshawi; hyd, hydei. Nine amino acids have been omitted from human $(\mathrm{h})$ or murine $(\mathrm{m})$ Hes6 for clarity. The CK2 phosphoacceptor is shown in red and critical acidic residues that are required for phosphorylation are shown in green. 


\section{Chapter 3}

Role for PP2A in Notch-mediated lateral inhibition. 


\section{Abstract:}

Accumulating evidence indicates that repression of Ato and the R8 fate by $\mathrm{E}(\mathrm{spl})-\mathrm{m} 8$ requires phosphorylation by protein kinase CK2. This role has been inferred from analysis of a CK2-phosphomimetic variant of $\mathrm{E}(\mathrm{spl})-\mathrm{M} 8$ and by the controlled expression of $R N A i$ constructs that target the catalytic subunit of CK2. In the bristle lineage, it has however, remained unclear whether repression is regulated by dephosphorylation. The studies described in this chapter extend earlier work describing opposing functions of CK2 and the phosphatase PP2A in the circadian clock. Using the bristle, we provide evidence that neural repression by ectopic M8, indeed, depends on endogenous levels of CK2. In addition, we demonstrate that increased dosage of the PP2A, regulatory subunit, widerborst (wdb), elicits bristle and wing margin defects that closely mimic loss of Notch functions. These effects appear to be sensitive to CK2 levels. Given that Wdb confers substrate-specificity to PP2A, we have assessed for its activities in backgrounds mutant for Notch and $E(s p /)$. We demonstrate that $w d b$ gain- or loss-of-function elicit inverse effects on the eye defects of $N^{s p l}$, effects that involve the specification and differentiation of the 'founding' R8 photoreceptors. Similarly, wdb gain-of-function rescues the eye and R8 defects of $N^{s p l} /+; E(s p l) D /+$ flies, a genetic interaction that was instrumental in the identification of the $E(s p l) C$. Wdb overexpression also mitigates loss of the eye field elicited by a phosphomimetic variant of M8, raising the possibility that $\mathrm{PP} 2 \mathrm{~A}$ may target residues of $\mathrm{M} 8$ that are subject to secondary phosphorylation, and appear to be necessary for full repressor activity. Together, the studies identify that $w d b$ is a participatory regulatory subunit that functions during Notch signaling, and indicate that reversible phosphorylation influences $\mathrm{E}(\mathrm{spl})$ activity. 


\section{Introduction:}

During neurogenesis, Notch-mediated lateral inhibition regulates the dichotomy of neural versus epidermal cell fate and is vital for proper patterning of sensory organs (Artavanis-Tsakonas et al., 1999; Blaumuller and Artavanis-Tsakonas, 1997; Bray, 2006; Gibert and Simpson, 2003). The process of lateral inhibition has been particularly well characterized during patterning of two sensory organs, the Drosophila compound eye and the mechanosensory bristles such as the machrochaetes (MC's), the microchaetes (mc's) and the interommatidial bristles (IOB's) (Modolell, 1997; Modolell and Campuzano, 1998; Simpson, 1990).

The adult Drosophila eye is characterized by the arrangement of facets (ommatidia) in a precise hexagonal pattern. In the developing retina, a wave of cell specification and differentiation called the morphogenetic furrow (MF), progresses from the posterior to the anterior of the eye disc and in its wake photoreceptors are specified in an ordered manner (Wolff and Ready, 1991). The first cell type to be specified in the eye disc is the R8 photoreceptor (Jarman et al., 1994). Because the R8's recruit all secondary photoreceptors of the ommatidia, this cell type is commonly referred to as the 'founding photoreceptor'. The hexagonal patterning of the adult compound eye, to a large extent, depends upon the precise positioning of R8 cells in the eye disc (Cagan and Ready, 1989a; Cagan and Ready, 1989b). It is likely that the mechanism of R8 patterning is more general to other developmental contexts as well. It is, therefore, of interest and importance to better understand the processes governing the specification and the phasing of R8's. Analysis of mutants has shown that the loss of R8 specification leads to the loss of secondary photoreceptor recruitment and manifests as a reduced eye, whose severity is proportional to the number of R8's that are lost (Jarman et al., 1994). In contrast the specification of excess R8's results in aberrant secondary cell recruitment and manifests as a rough eye (White and Jarman, 2000). 
Both, the specification and number of R8's, requires a precise control over Notch pathway activity (Baker, 2001; Baker, 2002; Baker, 2004; Baker et al., 1990; Baker et al., 1996; Baker and Zitron, 1995; Lee et al., 2000; Ligoxygakis et al., 1998), which includes receptor processing, maturation, etc., outlined in details in Chapter 1.

Inhibitory Notch signaling also plays a role in the position and numbers of MC's, mc's and IOB's. All of these bristle types depend upon the proper positioning of a precursor cell, the bristle sensory organ precursor (SOP). Akin to the R8 cells, the loss of the SOP results in loss of MC's, mc's and IOB's (Giebel and Campos-Ortega, 1997; Nakao and Campos-Ortega, 1996). In contrast, excess SOP's result in their development as ectopic MC's, mc's and IOB's (Campos-Ortega, 1998). After its specification, the SOP undergoes a series of asymmetric cell divisions (see Chapter 1 ) to give rise to the socket, the shaft, the neuron and the sheath cells. The stoichiometric specification of these four cell types defines these mechanosensory organs. In essence, the specification of R8's, SOP's and the cells of the SOP lineage depend on Notch-mediated lateral inhibition.

Prior to lateral inhibition, group of cells in the eye and wing anlagen (from which the thorax arises), known as the pro-neural clusters (PNC's), express the proneural basic- helixloop-helix (bHLH) transcriptional activators encoded by atonal (ato) or the genes of the achaete-scute complex (ASC) (Jarman et al., 1995; Modolell and Campuzano, 1998). These proneural activators bestow neural competency to all cells of the PNC. During lateral inhibition, the presumptive neural precursor in the PNC, invariably a single cell, expresses the ligand Delta at a level sufficient to activate Notch signaling in the adjacent cells of the PNC. This leads to the expression of the bHLH repressors encoded by the Enhancer of split Complex, E(spl)C (Delidakis and Artavanis-Tsakonas, 1991; Klambt et al., 1989; Knust et al., 1992). The $\mathrm{E}(\mathrm{spl})$ repressors along with the obligate co-repressor Groucho (Chen and Courey, 2000; Paroush et al., 1994) antagonize Ato/ASC, thereby restricting the R8 or SOP fate to a single cell from each PNC. Thus a complex interplay between the proneural 
activators and the $\mathrm{E}(\mathrm{spl})$ repressors regulate the final outcome of neural cell fate specification. In accordance, overexpression of Ato/ASC or loss of $E(s p l)$ leads to neural hyperplasia, whereas increased $E(s p l)$ dosage or loss of Ato/ASC manifests as neural hypoplasia.

Given the importance of $E(s p l)$ in regulating Ato/ASC activities, a number of studies have focused on identifying the functional domains of these repressors and the mode of their regulation. A long standing view is that repression by $\mathrm{E}(\mathrm{spl})$ activity primarily reflects the transcription and accumulation of these proteins in the non-R8/SOP cells. This view is incomplete. Studies from our lab indicate that of the seven $\mathrm{E}(\mathrm{spl})$ repressors, three members, i.e., M8, M5 and M7, are phosphorylated by CK2 (Trott et al., 2001b). Of these three members, M8 is expressed in early eye development during R8 patterning (see below), and its phosphorylation by CK2 is required for repressor activity against Ato during R8 selection (Karandikar et al., 2004). Specifically, it was found that a CK2-specific phosphomimetic variant of $M 8\left(M 8 S^{159} D\right)$ elicits a severe reduced eye, a phenotype that is not seen upon overexpression of wild type $\mathrm{M} 8$ or $M 8 \mathrm{~S}^{159} \mathrm{~A}$, a variant that is refractory to CK2 (Karandikar et al., 2004). In the case of $M 8 S^{159} \mathrm{D}$, the reduced eye reflects the loss of the Ato-positive cells, i.e., the founding R8's. These effects of $M 8 S^{159} \mathrm{D}$ closely mimic those elicited by the $m 8$ allele $E(s p l) D$ in combination with $N^{s p l}$ (Nagel and Preiss, 1999). $E(s p l) D$ encodes a truncated protein called $M 8^{*}$ that lacks the $56 \mathrm{C}$-terminal residues $(\mathrm{CtD})$, including the $\mathrm{CK} 2$ phosphorylation and Gro-recruitment sites (Tietze et al., 1992). Because of its resultant inability to recruit Gro, $E(s p l) D$ is termed as Gro-independent hypermorph (Nagel et al., 1999). Moreover, it has been reported that M8SD and M8* exhibit an enhanced interaction with Atonal, as compared to that with M8 or M8SA (Karandikar et al., 2004). To account for the similar effects of M8SD and M8* on eye development, it has been proposed that nonphosphorylated M8 is autoinhibited in an intramolecular manner by its CtD. In this model, phosphorylation of the CtD would lead to its displacement, thereby enabling repression. This 
conformational restraint is bypassed by CtD deletion in $\mathrm{M}^{*}$. If so, phosphorylation would be integral to lateral inhibition by Notch, and indicate that CK2 plays a critical role in the regulation of Notch signaling.

Previous studies, employing CK2-RNAi and CK2 dominant negative $(D N)$ constructs have also enabled an assessment of the role of CK2 in lateral inhibition. Specifically, the loss of CK2 results in the specification of ectopic R8's and SOP's in the eye and wing disc, respectively (Bose et al., 2006). While these studies implicate a role for CK2 in Notchmediated lateral inhibition, not all $\mathrm{E}(\mathrm{spl})$ members are regulated by phosphorylation. The differential phosphorylation suggests that the conformation of individual $E(s p l)$ proteins is an important underlying structural feature that is essential for repression and may distinguish the activities of individual members. The ability of CK2 to elicit conformational changes would be consistent with the roles of phosphorylation in regulating rapid control over the activity of diverse proteins. In addition, to the highly conserved CK2 phosphorylation site, the CtD of M8 also conserves a number of additional serine residues. More recent studies from our lab suggest that phosphorylation at these secondary sites potentiates M8 activity. Given that Notch-mediated lateral inhibition is reiteratively employed in cell fate specification during eye and bristle development, the possibility arises that a precise control over repression by M8 involves reversible phosphorylation via phosphatases. A candidate phosphatase that has emerged is PP2A. This possibility is supported by studies that increased dosage of microtubule star ( $m t s)$, the catalytic subunit of the phosphatase PP2A, elicits ectopic bristles and notched wings akin to Notch loss of function (Kunttas-Tatli et al., 2009).

In the case of PP2A, its ability to dephosphorylate a target protein requires the association of specific regulatory subunits (for details see chapter 1 ). The studies described in this chapter provide more direct in vivo evidence that repression by M8 involves both 
phosphorylation and dephosphorylation. In addition, these studies identify a role for the PP2A B' regulatory subunit, widerborst, in Notch signaling.

\section{Results:}

\section{Loss of CK2 activity inhibits repression by M8:}

As described in Chapter 2, compromising CK2-activity leads to the specification of ectopic R8's and bristle SOP's, hallmarks of loss of E(spl) activity and impaired lateral inhibition. Moreover, these effects are exacerbated in an $E(s p l) D /+$ background, where the dosage of wild type M8 is halved. The possibility arises that these effects may reflect the hypophosphorylation of full length $M 8$ encoded by the $E(s p l)^{+}$allele. We reasoned that if phosphorylation by endogenous CK2 permits M8 activity, impairing CK2 levels should mitigate the effects of ectopic M8 on bristle development.

For these studies the scaGal4 enhancer trap was used, because it is active in the PNC's that give rise to the MC's, mc's and IOB's (Giebel and Campos-Ortega, 1997; Nakao and Campos-Ortega, 1996). As previously reported by others and us, ectopic M8 elicited a strong loss of all of these three bristle types (Fig. 1A, A'). The ability of ectopic M8 to elicit these effects was mitigated by the co-expression of a UAS-CK2-RNAi construct, in which case the specification of these three bristle types was restored (Fig. 1B, B'). To quantify the extent of this rescue we focused on the four scutellar MC's because they are number and position invariant. In contrast to the four MC's in wild type flies (not shown), ectopic M8 results in an average MC count of $0.7 \pm 0.4$ (Fig. $1 \mathrm{C}$ ), whereas co-expression of a CK2-RNAi construct

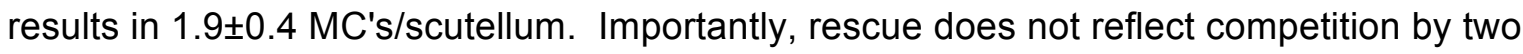
UAS-constructs for a rate-limiting amount of Gal4 produced by scaGal4, because coexpression of a UAS-LacZ construct did not mitigate the effects of ectopic M8 (data not shown). The expression of the CK2-RNAi construct on its own lead to ectopic scutellar MC's 
whose severity averaged $5.1 \pm 0.1$, as previously described (Bose et al., 2006). On their own, the Gal4 driver or the UAS lines for $m 8$ or CK2-RNAi do not affect the numbers/patterning of the MC's. An inverse test for the enhancement of the activity of ectopic M8 by a simultaneous increase in CK2 dosage was precluded by the absence of UAS-m8 lines that elicit marginal (weak) bristle loss.

Increased widerborst (wdb) dosage elicits bristle and wing defects akin to Notch loss of function:

It has remained unclear whether phosphatases oppose CK2 activity during inhibitory Notch signaling. A candidate phosphatase that has emerged as an attractive possibility is PP2A (Kunttas-Tatli et al., 2009), based on the observation that increased dosage of the PP2A catalytic subunit, $m t s$, elicits bristle, wing and retinal defects akin to Notch loss of function. In addition, coordinated activities between CK2 and PP2A appear to regulate the phosphorylation of Period, a central regulator of the Drosophila clock (Sathyanarayanan et al., 2004). Therefore, the possiblity arises that a similar antagonism between CK2 and PP2A might operate in the regulation of M8 activity.

We focused on the B' regulatory subunit of PP2A, encoded by widerborst (wdb), since it had been identified in a gain of function screen for genes that affect the Drosophila adult peripheral nervous system (Abdelilah-Seyfried et al., 2000). In this screen, scaGal4 driven expression of UAS-wdb (wdb ${ }^{E P 3559}$ ) elicited ectopic and split MC defects. However, no follow up studies have been conducted to define the underlying mechanisms. Based on these studies and the possible antagonism between CK2 and PP2A, we tested if the bristle defects of UAS-wdb are modulated in a CK2 gain/loss of function background, but found this to not be the case (data not shown). Given that $s c a G a / 4 /+;$ UAS-wdb/+ flies display bristle defects with a strong and complete penetrance, the possibility arose that this is a limit phenotype that is not amenable to modulation. Therefore, we used a weaker driver, 109-68Gal4 that is active in 
PNC's (Kahali et al., 2009; Kahali et al., 2010).

We find that 109-68Ga/4/+; UAS-wdb/+ flies display ectopic and split MC's, and a mild loss of the IOB's, but did not affect the hexagonal patterning or the size of the adult eye (Fig. $\left.2 \mathrm{~A}, \mathrm{~B}, \mathrm{C}, \mathrm{C}^{\prime}\right)$. This indicates that the threshold requirement of $\mathrm{Wdb}$ to perturb the eye is more than that of the bristles. However, we predicted that if the antagonism between CK2 and PP2A involves direct interactions, the MC defects of $w d b$ should be sensitive to CK2 dosage. Indeed, we find this to be the case. While 50\% 109-68/+; UAS-wdb/+ flies, display split MC's (Fig. 2D), the co-expression of a UAS-CK2 construct suppressed this defect, whereas it was enhanced in a background with reduced CK2 dosage (CK2-RNAi, see Fig. 2D). Importantly, the co-expression of a UAS-Tik construct was ineffective at mitigating the effects of ectopic Wdb. Because Tik harbors two mutations, one of which, $E^{165} \mathrm{D}$ perturbs a putative PP2A binding site, raising the possibility that the CK2-PP2A interaction may be required to downregulate phosphatase activity. Expression of a UAS-LacZ construct did not modulate the MC defects of 109-68/+; UAS-wdb/+ flies (data not shown) indicating that the suppression by UAS-CK2 is not due to competition by two UAS-constructs for rate limiting amount of Gal4. Moreover, none of the UAS-lines, by themselves, exhibit split MC's (data not shown), indicating that the effects we see may reflect interactions between PP2A and CK2.

\section{Wdb overexpression elicits Notched wings:}

To independently evaluate the role of $w d b$ in Notch signaling we used $E(s p l) G a l 4$. We find that expression of $w d b$ elicits notched wings with a high penetrance (Fig. 2E), but did not elicit any bristle defects (data not shown) since the enhancer trap presumably mimicks some but not all expression domains of endogenous $E(s p l)$. Together, the bristle and wing defects of UAS-wdb that we find are akin to those recently reported upon expression of the PP2A catalytic subunit, UAS-mts with either 109-68Gal4 or E(spl)Gal4 (Kunttas-Tatli et al., 2009). Thus $w d b$ and $m t s$ activities appear to occur in similar developmental contexts, and the likelihood is high that Wdb may positively regulate PP2A activity during Notch signaling. 
Wdb dosage modulates the retinal defects of $\boldsymbol{N}^{s p l}$ :

Lateral inhibition during SOP or R8 selection involves similar mechanisms and appears to also involve CK2 (Chapter 2, see above). While a decrease in CK2 levels impairs lateral inhibition and results in specification of ectopic SOP's/R8's, increased dosage is without effect. Given this, the lack of an effect of ectopic Wdb on the hexagonal architecture of the adult eye (Fig. $2 \mathrm{C}$ ) raised the possibility that the threshold requirements for this phosphatase might be different in the case of R8 selection from those in the SOP.

To evaluate the possibility that $w d b$ does play a role in $\mathrm{R} 8$ selection, we employed the Notch mutation, $N^{s p l}$. Even though Notch is required in multiple developmental contexts, the $N^{s p l}$ mutation only affects eye and bristle development, indicating that it perturbs the threshold requirement for Notch in these developmental contexts, than others. Specifically, the $N^{\text {spl }}$ mutation increases receptor sensitivity to Delta, thereby allowing inappropriate Notch activity in R8 precursors (Li et al., 2003), resulting in the loss of R8's. Because the morphogen Dpp, which is secreted by differentiated R8's, is required for atonal expression anterior to the MF (Baonza and Freeman, 2001), further R8 specification becomes abrogated. This leads to an overall reduction in facet number and a loss of ommatidial phasing, which manifest as reduced and uniformly rough eyes, respectively (Fig. 3A). Consistent with $N^{\text {spl }}$ rendering R8's sensitive to inhibitory Notch signaling, its eye defects are rescued by decreased dosage of $E(s p l)$ repressors (Parks et al., 1995). In addition, conditions that should result in the hypophosphorylation of M8 are expected to impair M8 activity. If so, these should lead to the suppression of the retinal defects of $N^{\text {spl }}$. Consistent with this reasoning, a targeted reduction of CK2 functions (CK2-RNAi) or Mts gain of function (UAS-mts) suppresses the retinal defects of $N^{\text {spl }}$ (Kahali et al., 2009; Kunttas-Tatli et al., 2009). $N^{\text {spl }}$ therefore appears to provide a sensitized background where modulation of endogenous $\mathrm{E}(\mathrm{spl})$ activity by CK2 or PP2A can be easily assessed.

We therefore assessed whether $w d b$ recapitulates the effects of $m t s$ during R8 
development, as it would further implicate wdb as a participating regulatory subunit. To assess restoration of the eye and R8 defects inherent to $N^{s p l}$, we used the enhancer trap $h^{H 10} \mathrm{Gal}$, previously used by others and us (Huang and Fischer-Vize, 1996; Kahali et al., 2010) to drive transgene expression anterior to the MF. In contrast to the reduced and rough eye of $N^{s p l}$ males (Fig. 3A), expression of UAS-wdb increases the size of the eye field and appears to also restore ommatidial phasing (Fig. 3B). Furthermore, UAS-wdb expression also suppresses the ectopic and the missing IOB defects of $N^{s p l}$ and restores patterning of the IOB's at alternating positions in the ommatidial lattice (compare Fig. $3 A^{\prime}, B^{\prime}$ ) akin to that in wild type flies (not shown). To further evaluate these findings we tested $w d b$ with additional Gal4 drivers whose expression relative to the MF is well defined (Bessa et al., 2002). Expression of $w d b$ with 109-68Gal4 (within the MF) also rescued the reduced and rough eye of $N^{s p l}$ to a level that was similar to that upon its expression with $h^{\text {H10Gal4 }}$ (Fig. 3D). Expression of $w d b$ with gmrGal4 (posterior to the MF, after R8 patterning) had modest effects on the reduced and rough eye of $N^{\text {spl }}$ (data not shown). At present the reason for this modest suppression is unclear. Even though wdb is known to play a role in apoptosis (Schreiber et al., 2002), the partial rescue by ectopic Wdb is unlikely to reflect attenuated apoptosis because overexpression of the inhibitor of apoptosis p35 does not rescue the eye defects of $N^{\text {spl }}$ ( Li et al., 2003).

Given that PP2A gain of function suppresses the retinal defects of $N^{s p l}$, we next tested whether PP2A loss of function exacerbates the retinal defects of $N^{s p l}$. We tested the recessive lethal loss of function allele $m t^{\text {XE2258 }}$ (Wassarman et al., 1996) and found that it did not exacerbate the reduced eye of $N^{s p l}$. This result indicates that a $50 \%$ reduction in PP2A catalytic activity is not rate limiting for phosphatase activity. Given that increased dosage of $w d b$ suppresses the retinal defects of $N^{s p l}$, we tested for enhancement by $w d b$ loss of function. Indeed, $w d b^{K G 02997}$, a hypomorph (Bellen et al., 2004), exacerbated the rough and reduced eye of $N^{\text {spl }}$ (Fig. 3C). This effect only occured when $w d b^{K G 02997}$ was homozygous (Fig. 3C). In 
addition, enhanced IOB patterning defects were found in $N^{s p /} / Y ; w d b^{K G 02997} / w d b^{K G 02997}$ flies (Fig. 3C) where multiple IOB's are found at normal and ectopic positions in the ommatidial lattice. Homozyous $w d b^{K G O 2977}$ flies, on their own, do not exhibit facet or IOB defects (data not shown). To determine specificity, we tested a homozygous lethal mutant of twins, tws ${ }^{02412}$ (Wang et al., 2009), but found that it did not modulate $N^{\text {spl }}$. Mutations in the other regulatory subunits (B56-1 and B") could not be tested in a similar manner, as these are currently unavailable.

To quantify the modulation of $N^{s p l}$, we determined facet counts, an approach used to determine eye size (Kahali et al., 2009; Kahali et al., 2010; Kunttas-Tatli et al., 2009; Nagel et al., 1999; Shepard et al., 1989). For each genotype, facet numbers from $\geq 10$ flies were quantified. The baseline facet number for $N^{\text {spl }}$ males is $\sim 320 \pm 10$ (Fig. 3D). In contrast, expression of $w d b$ with $h^{H 10 G}$ and $109-68 G a l 4$ significantly increases the facet count to $500 \pm 10$ and $480 \pm 11$, respectively (Fig. 3D). As controls, we also analyzed $N^{\text {spl }}$ in combination $h^{H 10} \mathrm{Gal} 4$ or $109-68 \mathrm{Gal} 4$, but found that these flies display facet numbers $(330 \pm 10)$ that are indistinguishable from those in $N^{\text {spl }}$ (Fig. 3D). The absence of an effect indicates that rescue requires $w d b$ overexpression. Consistent with the adult eye, $N^{s p /} / Y ; w d b^{K G 02997} / w d b^{K G 02997}$ flies display a significantly reduced facet count of $\sim 200 \pm 10$ (Fig. 3D). A single dose of $w d b^{K G 02997}$ in combination with $N^{s p l}$ displayed facet numbers similar to $N^{s p l}$ (Fig 3D). The opposite effects of wdb gain and loss of function on $N^{\text {spl }}$ strengthen the potential role of this regulatory subunit in Notch signaling.

\section{$W d b$ effects on $N^{s p l}$ reflect $R 8$ specification.}

We next sought to determine if rescue of $N^{\text {spl }}$ by ectopic Wdb reflects restored $\mathrm{R} 8$ specification. We thus stained third instar larval eye discs for Senseless (an R8 specific marker) and Elav (a pan-neuronal marker). In wild type discs (Fig. 4A) Sens expression demarcates the initiation of R8 differentiation (Frankfort et al., 2001; Nolo et al., 2000), following which Sens levels are maintained throughout the eye field. In addition, these Sens+ 
cells are surrounded by Elav+ cells, whose numbers systematically increase towards the posterior margin of the eye disc. This increase in Elav+ cell numbers reflects the recruitment of the secondary (R1-R7) photoreceptors. In $N^{\text {spl }}$ eye discs (Fig. 4B) Sens expression is not uniform along the dorso-ventral axis and is not sustained along the antero-posterior (AP) axis indicating that the inappropriate Notch activity impairs proper R8 differentiation. Consistent with this, the number of Elav+ cells does not systematically rise towards the posterior margin of the eye field. Moreover, a number of R8 cells fail to recruit secondary photoreceptors and display twinning. These effects are rescued by overexpression of Wdb. Ectopic Wdb enhances Sens expression in the MF and these cells appear to be patterned in a manner closer to that of wild type disc (Fig. 4C). The earlier expressivity of Sens in the presumptive R8 equivalence group at the leading edge of the MF may indicate that enhanced PP2A activity leads to diminishing M8 activity. If so, Ato activity in the equivalence group would be enhanced leading to upregulation of sens expression (Jafar-Nejad et al., 2003). Moreover, these Sens+ cells appear to recruit secondary photoreceptors with greater efficiency. Thus ectopic Wdb (enhanced PP2A activity) appears to have effects similar to the reduction of $\mathrm{E}(\mathrm{spl})$ dosage/activity (Shepard et al., 1989). In contrast $w d b^{K G 02977}$ enhances the R8 defects of $N^{s p l}$. In this case (Fig. 4D), Sens expression is further diminished and appears to not be sustained along the AP axis. Consequently, significant regions of the eye disc display a complete absence of R8's or secondary photoreceptors, and the spacing of these (Sens+ and Elav+) clusters appears to be more dispersed than in $N^{s p l}$ discs. The possibility thus arises that attenuated PP2A activity potentiates inhibitory Notch signaling and that the effects of wdb on the adult eye reflect R8 specific effects. In summary, UAS-wdb suppresses, whereas $w d b^{K G 02977}$ enhances the defects of $N^{\text {spl }}$ during R8 specification, differentiation and secondary photoreceptor recruitment.

\section{$U A S-w d b$ rescues the reduced eye in $N^{s p l} /+; E(s p l) D /+$ females:}

$N^{s p l}$ has been the subject of screens to identify enhancers and suppressors (Brand and 
Campos-Ortega, 1990). Surprisingly, neither $m t s$ nor $w d b$ have been identified in these studies. Our findings on the effects of $w d b$ indicate a potential role for PP2A in Notchmediated R8 selection. Given the novelty of this finding, we decided to independently test its effects in a $E(s p l)$ mutant background. For this study we employed the dominant $m 8$ mutation $E(s p l) D$. As mentioned above (see Chapter 1), $E(s p l) D$ severely enhances the reduced and rough eye in $N^{s p l}$ males, but, on its own, does not exhibit eye or bristle defects (Nagel and Preiss, 1999; Tietze et al., 1992). Given the recessive nature of the $N^{s p l}$ mutation, $N^{s p l} Y$; $E(s p l) D /+$ flies display a severely reduced eye with $<15$ facets, whereas that of $N^{s p l} /+$; $E(s p l) D /+$ (females) is more attenuated ( 250 facets, Fig. 5B, (Nagel and Preiss, 1999)). The modest effect in females raises the prospect that this is not a limit phenotype (Nagel et al., 1999), and one more amenable to modulation. Consistent with this reasoning, the reduced eye of $N^{s p l} /+; E(s p l) D /+$ is enhanced by halved dosage of ato or its heteromeric partner, daughterless $(D a)$, whereas it is suppressed by mutations in Delta that decreases Notch activity in R8 precursors (Nagel and Preiss, 1999; Shepard et al., 1989). Therefore, the retinal defects of $N^{s p l} /+; E(s p l) D /+$ are sensitive to proneural dosage and to a decrease in Notch activity. Therefore we predicted that attenuating phosphorylation of M8, either by decreased CK2 activity or enhanced PP2A activity should impair repressor activity leading to a rescue of the reduced eye of $N^{s p l} /+; E(s p l) D /+$ flies.

We find that overexpression of $W d b$ rescues the reduced eye of $N^{s p l} /+; E(s p l) D /+$ flies and appears to restore facet phasing (Fig. 5C). Quantification of the facet numbers indicates that ectopic Wdb significantly increases eye size (Fig. 5D). These effects require the ectopic expression of $\mathrm{Wdb}$, because no rescue is observed in the absence of a UAS-wdb construct or in other control genotypes (Fig. 5D). We next determined whether suppression involves a rescue of R8 specification and differentiation. Compared to WT (not shown) we find that in $N^{s p l} /+; E(s p l) D /+$ eye discs, there are numerous gaps between the specified and differentiated R8's, and these regions are devoid of secondary photoreceptors (Fig. 5B'). In contrast, 
expression of UAS-wdb in $N^{s p l} /+; E(s p l) D /+$ females increases the number of Sens+ R8's, restores the spacing between R8's, and enhances the number of Elav+ clusters (Fig. 5C'). Therefore rescue of the reduced eye of $N^{s p l} /+; E(s p l) D /+$ by increased dosage of $W d b$ reflects restored $\mathrm{R} 8$ specification and differentiation.

\section{Wdb gain of function suppresses the reduced eye of M8SD:}

We next sought to assess if M8 is an in vivo target for the PP2A holoenzyme containing Wdb. In the case of M8, its ectopic expression led to loss of the scutellar MC's, but this defect was not rescued by increased dosage of Wdb (data not shown). The possibility remains that a higher threshold of $w d b$ is required to modulate the effects of M8. As an alternative, we tested if ectopic Wdb can suppress the reduced eye of M8SD. For these studies, the weaker driver 109-68Gal4 was used to express a UAS-m8SD construct because the reduced eye is of moderate severity (Fig. 6B, (Karandikar et al., 2004)). In addition, 10968Gal4/+; UAS-m8SD/+ eye are uniformly rough and ommatidia are of variable size because secondary photoreceptor recruitment is suboptimal. These eye defects are rescued by increased dosage of $w d b$ (Fig. 6C). We quantified the extent of suppression with facet counts and found that 109-68Gal4/+; UAS-m8SD/+ flies display 350 10 facets, while in 109-68/+; UAS-m8SD/UAS-wdb flies that facet count increased to $\sim 500 \pm 15$ (Fig. 6D), indicating restoration of the eye field. On the other hand, the simultaneous expression of a UAS-lacZ construct did not modulate the facet counts of 109-68/+; UAS-m8SD/+ (Fig. 6D). This indicates that suppression of retinal defects by ectopic $\mathrm{Wdb}$ is not an effect of competition between two UAS-lines for Gal4.

To further assess the effects of Wdb on M8SD, we stained the corresponding eye discs with Sens and Elav. In 109-68/+; UAS-m8SD/+ eye discs, R8's are mispatterned and do not sustain Sens expression (Fig. 6B'). Consequently these compromised R8's inefficiently recruit secondary photoreceptors (Fig. 6B' inset). These R8 defects are strongly rescued by co-expression of Wdb (Fig. 6C'). Importantly, in discs co-expressing Wdb patterning of R8's 
appears closer to that in wild type (data not shown) or in discs only overexpressing Wdb (Fig. $\left.6 A^{\prime}\right)$. This effect does not reflect competition of two UAS-constructs because co-expression of LacZ is without effect (data not shown).

The findings that ectopic Wdb rescues the eye and R8 defects of $N^{s p l}$ males, $N^{s p l} /+$; $E(s p l) D /+$ females, and 109-68/+; UAS-m8SD/+ flies together indicate that Wdb is likely to contribute to PP2A activity that opposes repression by M8.

\section{Discussion:}

$\mathrm{E}(\mathrm{spl})$ repressors are the final effectors of inhibitory Notch signaling during which they antagonize transcriptional activities of the proneural activators, Ato and ASC. This antagonism, termed lateral inhibition (Lehmann et al., 1983; Simpson, 1990), was first thought to be exclusive to neural patterning, but later studies have uncovered more general roles in other developmental contexts where the selection of a single cell from a cluster/pool of equipotent progenitors is necessary for tissue patterning. It is therefore of importance to fully describe the underlying mechanisms.

Earlier studies focused on the specification and patterning of the bristle sensory organ precursors (SOP's). These studies established that loss of $\mathrm{E}(\mathrm{spl})$ repressors led to pervasive ASC activity in PNC's leading to the specification of ectopic SOP's and macrochaetes (MC's), whereas overexpression of $\mathrm{E}(\mathrm{spl})$ members led to ablation of the SOP's and the MC's (Campos-Ortega, 1998; Giebel and Campos-Ortega, 1997; Nakao and Campos-Ortega, 1996; Skeath and Carroll, 1991; Tata and Hartley, 1995). Consequently, it has been generally thought that transcription and accumulation of $E(s p l)$ repressors during lateral inhibition was, by itself, sufficient to antagonize ASC proteins during SOP selection, and that the $E(s p l)$ proteins play a redundant role in this process. Neither of these appears to be the case. In the eye, for example, the selection of a single R8 from a cluster of 'pre-R8' cells requires $E(s p l)$ activity, because loss of these repressors impairs lateral inhibition and leads to the 
specification of excess R8's. However, ectopic expression of M8 does not lead to exacerbated loss of R8's, as is the case in the SOP, even though endogenous $m 8$ is expressed during R8 selection (Giebel and Campos-Ortega, 1997; Karandikar et al., 2004). The most revealing insights into this conundrum have emerged from the R8 and eye defects of a unique dominant allele of $m 8$ called $E(s p l) D$ (Nagel et al., 1999; Nagel and Preiss, 1999). This allele encodes a truncated protein called $\mathrm{M}^{*}$ that lacks the 56 residue $\mathrm{C}$-terminal domain (CtD). Given the inactivity of full-length M8 and the hyperactivity of M8*, the late Dr. Jose Campos-Ortega presciently proposed that the C-terminus of M8 might be important in regulating M8 activity, but the underlying mechanism remained elusive.

Studies conducted in our laboratory uncovered that CK2 phosphorylates M8 in its CtD. It was subsequently shown that a variant of M8 harboring a phosphomimetic Asp residue at the CK2 site (M8-S $\left.{ }^{159} \mathrm{D}\right)$ ablated R8 specification and eye development (Karandikar et al., 2004), effects that were of a potency virtually identical to that of M8*. It was thus proposed that non-phosphorylated M8 is 'autoinhibited' by its CtD, and that phosphorylation would elicit CtD displacement to enable repression. The possibility thus arose that repression by M8 is regulated, and not simply the outcome of its accumulation in response to Notch signaling. Regulation of protein activities by phosphorylation is a widespread mechanism, and reflects the need for rapid switching between two conformational states. Consequently, these modifications are often, if not always, subject to fine-tuning, a function provided by the coordinated activities of phosphatases. One such phosphatase is PP2A, whose role in Notch signaling was first uncovered in a gain-of-function screen (Abdelilah-Seyfried et al., 2000), but was not followed-up in detail. It was subsequently shown by our laboratory that $m t s$, the unique catalytic subunit of Drosophila PP2A, is required for Notch signaling, and that CK2 and PP2A play opposing roles (Kunttas-Tatli et al., 2009), the former potentiating this process and the latter opposing it. However, the specific regulatory subunit that mediates the effects of PP2A remained to be identified, an aspect of importance because of their obligate 
requirement for recognition and dephosphorylation of target proteins (Eichhorn et al., 2009; Janssens and Goris, 2001; Silverstein et al., 2002; Virshup and Shenolikar, 2009).

In the studies reported here we have characterized the role for the PP2A-B' regulatory subunit, widerborst ( $w d b)$ in Notch-mediated lateral inhibition that further support the opposite functions of CK2 and PP2A. To assess its roles in this process, we have used Wdb gain/lossof-function analysis in wild type and $N$ and $E(s p l)$ mutant backgrounds. Together, the bristle, wing margin and eye analysis support the possibility that PP2A opposes inhibitory Notch signaling, that its effects may involve interactions with $\mathrm{CK} 2$, and that its target may be the M8 protein.

Our results demonstrate that the bristle loss phenotype of ectopic M8 is mitigated by a simultaneous reduction of CK2 levels/activity (Fig. 1). Thus CK2 is required for repression even in the SOP lineage, arguing against the possibility that phosphorylation is an eye/R8specific requirement (Karandikar et al., 2004). Of particular interest is our finding that the split and ectopic bristle defects of Wdb-gof (Fig. 2) are suppressed by co-expression of wild type CK2, but not by Tik, and modestly exacerbated in a CK2 compromised background. The lack of an effect of Tik is noteworthy because this variant of CK2 harbors an $E^{165} D$ mutation in a motif, HENRKL, previously proposed to mediate interaction of human CK2 with PP2A (Heriche et al., 1997). It may thus be the case that increased dosage of wild type CK2 displaces the regulatory Wdb subunit, thereby shifting the dynamics in favor of an inactive PP2A heterotrimer. Therefore, CK2 might oppose the effects of Wdb not only by antagonizing dephosphorylation of M8, but also by directly interacting with the PP2A core enzyme and inhibiting its activity. Further studies would be required to parse out the dynamics between PP2A holoenzyme and CK2 in the regulation of M8 activity. The notched wing phenotype and loss of wing margin bristles of flies overexpressing Wdb mirrors that upon overexpression of UAS-mts previously reported by us (Kunttas-Tatli et al., 2009). This phenotype is a hallmark of Notch loss of function mutants, supporting our proposal that a CK2-PP2A nexus is a 
component of Notch signaling.

Our studies in $N$ and $E(s p l)$ mutant backgrounds further support the proposed role for PP2A. As stated earlier, the eye defects of $N^{\text {spl }}$ result from increased Notch activity in R8 precursors (Li et al., 2003). This inappropriate Notch activity impairs the sustained expression of Sens, a transcription factor that is essential for R8 differentiation (Frankfort et al., 2001; Nolo et al., 2000). As a result, these R8's either lose Sens expression altogether leading to their apoptosis, or compromises their ability to recruit secondary photoreceptors. The outcome of these aberrations is a reduction of the eye size and the presence of ommatidia of varying size. Our finding on modulation of the eye defects of $N^{s p l}$ further support a role for Wdb. Increased $w d b$ dosage suppresses, whereas $w d b$ loss of function exacerbates the eye defects of $N^{\text {spl }}$ (Fig. 3). Importantly, the genetic interaction between $w d b^{K G 02977}$ and $N^{\text {spl }}$ provides direct genetic evidence of Wdb's role in Notch signaling. Moreover, Wdb-gof increases Sens levels and restores the hexagonal patterning of R8 cells, effects that are opposite to those with wdb-lof. It has been previously shown that decreased dosage of $\mathrm{E}(\mathrm{spl})$ or CK2 rescue the eye/R8 defects of $N^{\text {spl }}$ (Kahali et al., 2009; Shepard et al., 1989), as does an increase in the dosage of mts, the PP2A catalytic subunit (Kunttas-Tatli et al., 2009). In light of these results, it is reasonable to propose that $\mathrm{Wdb}$ is a relevant participatory regulatory subunit that enhances PP2A activity. In the sensitized R8 cell, this enhanced PP2A activity might elicit the dephosphorylation of endogenous M8 to attenuate repression, as appears to be the case of rescue by a decrease in $E(s p l)$ dosage. Our studies on the eye/R8 defects of $N^{s p l} /+; E(s p l) D /+($ Fig. 5) are also consistent with a role in Notch signaling.

If $\mathrm{Wdb}$ does dephosphorylate M8 to antagonize repression, one might expect that repression by $M 8$ should be mitigated by an increase in $w d b$ dosage. However, these studies did not bear out. On the other hand, an increase in $w d b$ dosage did rescue the dominant retinal defects of ectopic M8SD (Fig. 6), a paradoxical finding, given that the eye and R8 defects of this dominant (CK2) phosphomimetic variant are insensitive to the dosage of $E(s p l)$ 
or CK2 (Kahali et al., 2009). More recent data (Kahali and Bidwai, In preparation) indicates that the $\mathrm{CtD}$ of M8 harbors additional serine residues that conform to the recognition consensus for three additional kinases, i.e., MAPK, CK1 and GSK3. Moreover, it appears to be the case that repression of Ato during R8 selection requires modification by both $\mathrm{CK} 2$ and MAPK, with the former kinase functioning at the head of this hierarchy. It would be reasonable to suggest that PP2A may target the MAPK site of $M 8$, as appears to be the case in other systems (Junttila et al., 2008; Silverstein et al., 2002; Wassarman et al., 1996). In vitro phosphatase assays will be required to define the site(s) on M8 that are targets of the PP2A holoenzyme containing Wdb.

Together, emerging data indicates that a complex intersection of kinases and phosphatase(s) influence Notch signaling. Given the consistency of these diverse findings, it will be important to determine how this process is fine-tuned during R8/SOP selection. One possibility is that this intersection may serve to 'filter' transcriptional noise, i.e., stochastic fluctuations in Ato/ASC expression. In the case of the R8 cell, lateral inhibition may initiate only when an R8 precursor accumulates Ato above the 'threshold' for R8 specification. If so, PP2A may serve to prevent precocious onset of repression by $M 8$, a regulation that is bypassed by the $E(s p l) D$ mutation. An alternative possibility, one that we favor, is that PP2A may function to rapidly de-activate M8 immediately after R8 selection has occurred. Following differentiation, the R8 cell recruits surrounding uncommitted cells as the R2/5 photoreceptors, a process that also involves Notch and $\mathrm{E}(\mathrm{spl})$ activity. However, it is currently unknown which of the $E(s p l)$ genes mediates this binary cell fate determination. Nevertheless, it may be the case that a rapid de-activation of M8 by PP2A may be necessary to permit other $\mathrm{E}(\mathrm{spl})$ proteins to allow for the proper specification of secondary photoreceptors, a full complement of which are necessary for vision. Our findings would be in-line with emerging data that Groucho, the obligate co-repressor for all $\mathrm{E}(\mathrm{spl})$ proteins, is itself regulated by the activities of multiple kinases (Hasson et al., 2005; Lee et al., 2009). 
Sequence analysis indicate that the sites for phosphorylation are conserved among four out of seven $E(\mathrm{spl})$ members, i.e., M8, M5, M7 and $M \gamma$ (see Fig. 7, chapter 2), raising the possibility that PP2A may be involved in the regulation of these proteins as well. Moreover, the mammalian homolog of M8, Hes6, also harbors similar phosphorylation sites, and whose modifications are necessary for its interactions with Hes1, although the role of PP2A remains to be defined in this system. Hes6 regulates transitions in retinal cell fate specification, and during development of the cerebral cortex, where progenitors give rise to the ordered specification of neurons (first), astrocytes (second) and oligodendrocytes (third). It has, in fact, been proposed (Belanger-Jasmin et al., 2007) that regulated Hes6 activity (phosphorylation) may underlie the timing of transitions from neurogenesis to astrocyte specification, during which Hes6 promotes the former and inhibits the latter (Belanger-Jasmin et al., 2007; Gratton et al., 2003). In Drosophila, it may be the case that phosphorylation of M8 serves to first pattern the R8's, with dephosphorylation essential for the specification of the functionally distinct R1-7 photoreceptors. Phosphorylation of Xenopus NeuroD by GSK3 also serves as a timer during retinal development (Marcus et al., 1998; Moore et al., 2002).

Control by phosphorylation would be significantly faster and more robust, as compared to that based solely upon transcription. The similar regulation of M8 and Hes6 makes it likely that controlled phosphorylation and dephosphorylation hierarchy influencing M8 conformational switching is relevant to mammals. Future studies to identify the site on M8 that is a target for PP2A, and whether Hes6 is similarly regulated by controlled dephosphorylation will be required to more fully describe the mechanism(s) by which phosphorylation and dephosphorylation act as 'timers' controlling the onset of repression.

\section{Experimental Procedures:}

Fly stocks: 
Flies were raised at $24^{\circ} \mathrm{C}$ on standard Yeast-Glucose medium. The Gal4 drivers were generously provided by other researchers or obtained from the Bloomington Stock Center (denoted by the prefix B). The Gal4 drivers that were used in these studies are 109-68Gal4 (Jarman and Ahmed, 1998), scaGal4 (Giebel and Campos-Ortega, 1997), ey3.5-Gal4(II) (B8220), E(spl)Gal4 (B8225) and $h^{\mathrm{H10}} \mathrm{Gal}$ (Huang and Fischer-Vize, 1996). The mutant lines $w d b^{K G 02977}(\mathrm{~B} 2977), N^{s p l}(\mathrm{~B} 118, \mathrm{~B} 182)$ and $E(s p l) D(\mathrm{~B} 2447)$ were obtained from the Bloomington Stock Centre. Amita Sehgal, Rob Jackson and Ravi Allada generously provided the following fly lines, $w d b^{E P 3559}, U A S-R N A i$ and UAS-Tik. The UAS-M8 and UAS-M8SD flies were generated previously in the laboratory (Karandikar et al., 2004).

\section{Fly crosses and phenotypes:}

All crosses were performed at $24^{\circ} \mathrm{C}$, unless indicated otherwise. Fly heads were passed through a graded alcohol series for 24 hours each (25-50-75-absolute). Finally, heads were passed through Hexamethyldisalizane, and mounted on EM stubs using carbon tape (Ted Pella). Fly heads were dried for 24 hours, sputter coated with gold, and examined with a JEOL-6400 scanning electron microscope at an accelerating voltage of $20 \mathrm{kV}$. Images were acquired, processed with Adobe Photoshop and collated in Adobe Illustrator. For bristle phenotypes, newly eclosed adults were photographed using a Nikon digital camera attached to a Leica MZ16 stereomicroscope. For quantitative analysis of facets, crosses were established in duplicates, and 10-20 adult flies for each genotype were scored using light microscopy and SEM images.

\section{Immunostaining:}

Imaginal discs were isolated from late third instar larvae and processed as described with modifications. Discs were fixed in freshly prepared $4 \%$ paraformaldehyde in $1 \mathrm{x}$ phosphate buffered saline (PBS) for 45 minutes at $4^{\circ} \mathrm{C}$, and washed three times with PBS containing $0.3 \%$ Triton X-100 (PBS-TX). The discs were incubated for 12 hours at $4^{\circ} \mathrm{C}$ in PBS-TX containing 5\% normal goat serum and primary antibody, washed three times with 
PBS containing $0.3 \%$ Triton X-100 (PBS-TX) and immunostained for 2-3 hrs in secondary antibody. Following this, eye discs are washed three times with PBS containing $0.3 \%$ Triton X-100 (PBS-TX) and mounted in Vectashield. The following antibodies were used in this study guinea pig anti-Sens (gift of Hugo Bellen) at a dilution of 1:500 and mouse anti-Elav (DSHB) at a dilution of 1:500. Secondary antibodies (Molecular Probes) were goat-anti mouse-IgG coupled to Alexa Fluor 594 (1:1000) and goat anti-guinea pig-IgG coupled to Alexa Flour 594 (1:1000).

\section{Confocal microscopy:}

An Olympus FluoView (FV1000) was used for confocal imaging. Images in Figs. 4, 5 and 6 were generated from scans acquired every $1 \mu \mathrm{m}$ along the apicobasal axis of the discs. The scanning was limited along the Z-axis to acquire full spectral output of the fluorophores. Fluorophores were excited using appropriate excitation wavelengths. Individual Z-axis images were acquired, compressed as a Z-stack, and exported as TIFF files that were processed in Adobe Photoshop and collated in Adobe Illustrator. 


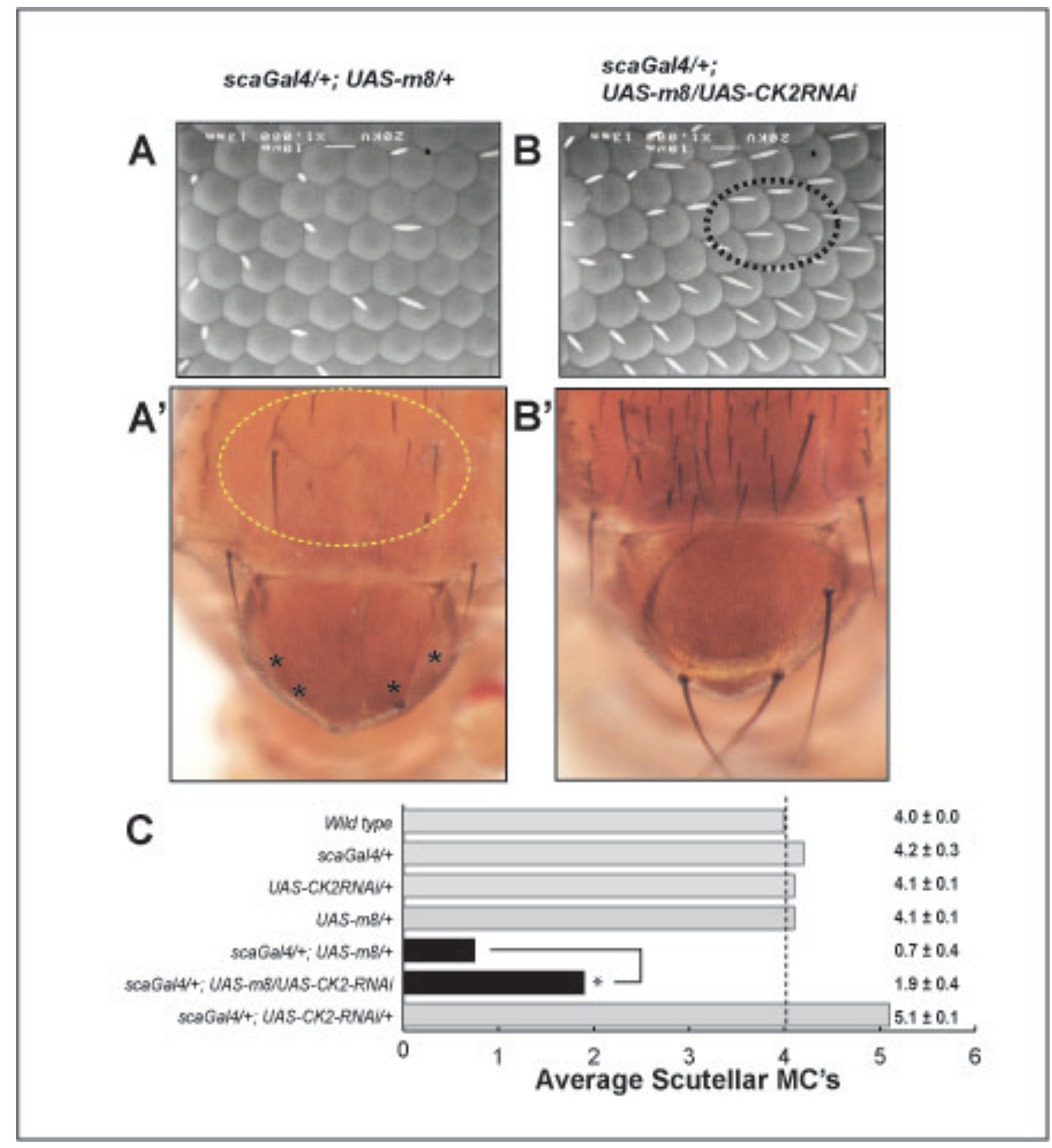

Fig.1. CK2-RNAi suppresses the bristle defects of ectopic M8. (A, B) Suppression of the IOB defects of scaGal4/+; UAS-m8/+ upon co-expression of UAS-CK2-RNAi. Dotted circle denotes restored IOB's. (A') Nota of flies expressing M8 alone. Yellow dotted circle denotes loss of mc's. Star denotes loss of scutellar MC's. (B') Nota of UAS-m8 + UAS-CK2-RNAi. (C) Suppression of the MC defects of $s c a G a / 4 /+;$ UAS-m8/+. Control genotypes (grey bars). Bristle defects were determined in $\geq$ 100 flies for each genotype. Solid line indicates genotypes compared. Asterisk denotes $P$-values $<$ 0.01 . Numbers to the right denote average scutellar MC counts. Dotted line denotes the wild type baseline for the MC counts. 


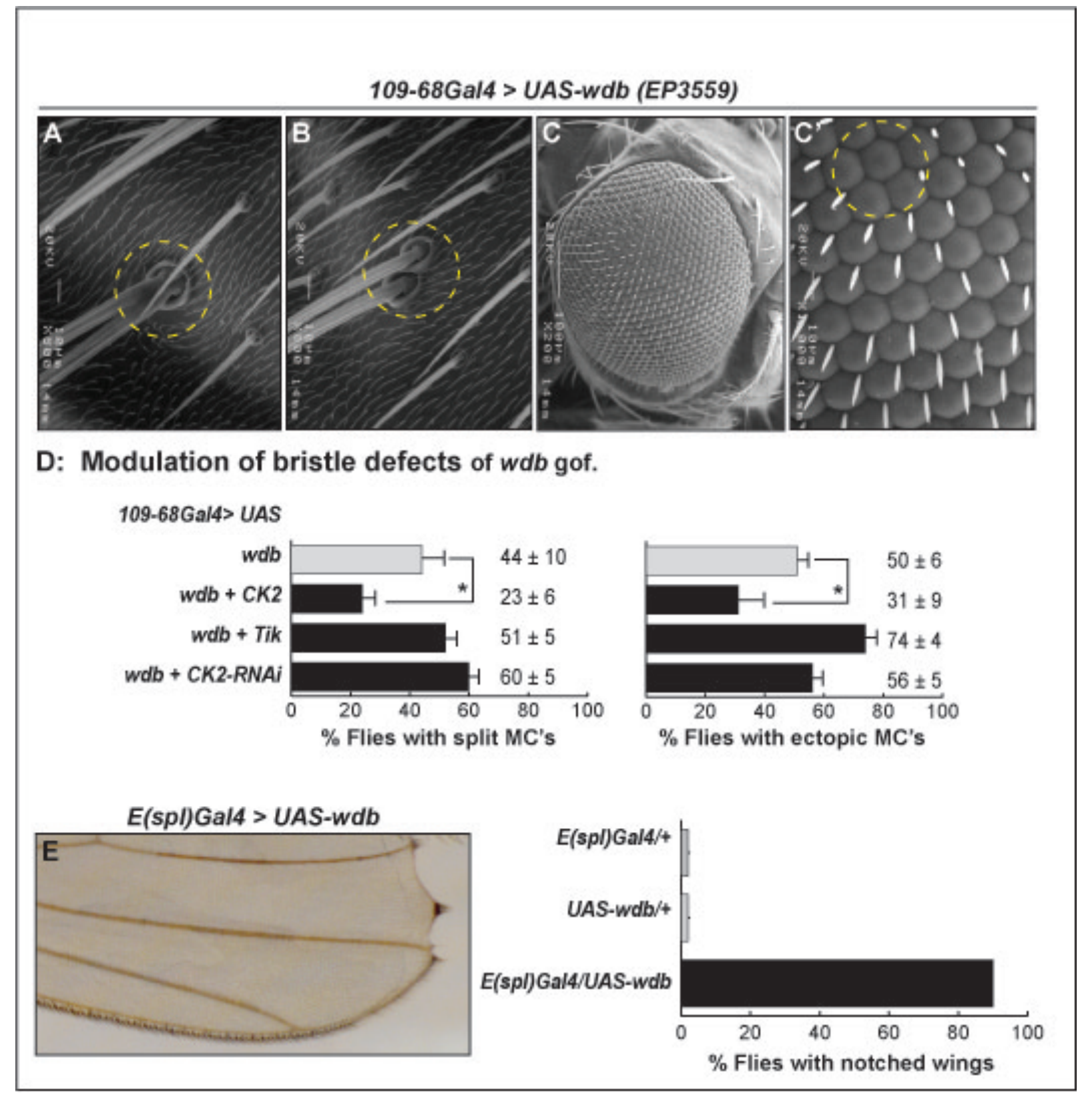

Fig. 2. Increased dosage of $w d b$ elicits bristle and wing defects akin to Notch loss of function.

Overexpression of $w d b$ in $N^{+}$flies elicit split MC's (A), ectopic MC's (B) and loss of IOB's (C, C'). Yellow dotted circle denotes the bristle defects. (D) CK2 dosage modulates the bristle defects of ectopic Wdb. Numbers to the right denote percent flies. Bristle defects were determined in $\geq 100$ flies of each indicated genotype. The solid line denotes compared genotypes. Asterisk denotes $P$ value $<0.01$ (E) Notched wing defects are elicited upon expression of UAS-wdb with $E(s p l) G a l 4$. Graph denotes percent flies with notched wings. 


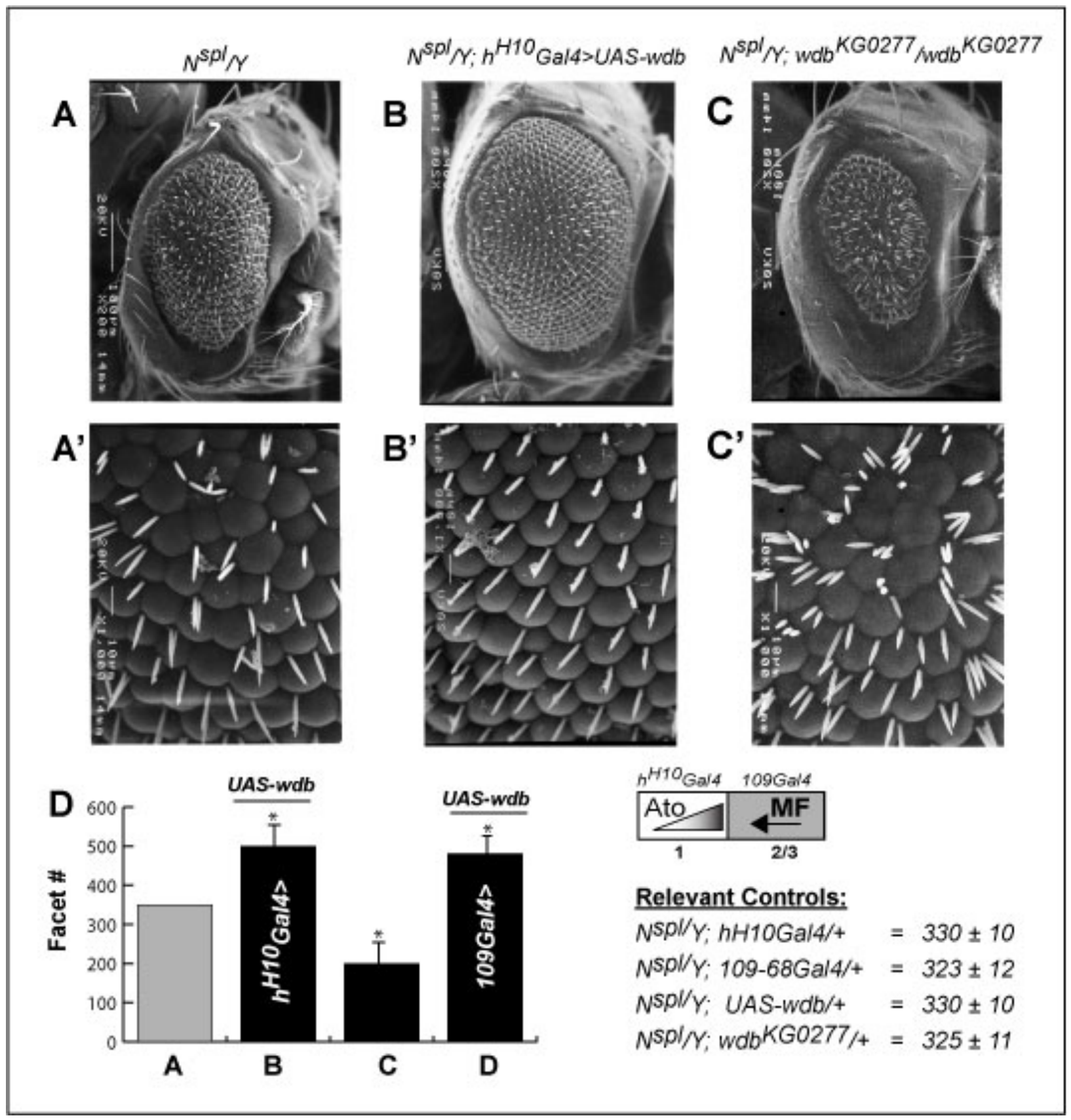

Fig. 3. Wdb modulates the retinal defects in $\boldsymbol{N}^{\text {spl }} / \boldsymbol{Y}$. (A-C') Adult eye phenotypes. The rough and reduced eye of $N^{s p l} / Y\left(\mathrm{~A}, \mathrm{~A}^{\prime}\right)$. Increased wdb dosage rescues the retinal defects of $N^{\text {spl }} / Y\left(\mathrm{~B}, \mathrm{~B}^{\prime}\right)$. Decrease in wdb dosage exacerbates the retinal defects of $N^{s p l} / Y\left(C, C^{\prime}\right)$. Anterior of the eye is to the right. Magnification 200X (A, B, C) and 1000X (A', B', C'). (D) Ommatidial (facet) counts were determined in $\geq 10$ flies of the indicated genotypes. Asterixes denote $P$-values $<0.01$. Inset in panel $D$ shows a schematic of the MF, the arrow denotes MF progression. 109-68Gal4 drives expression in the MF and $H^{h 10}$ Gal4 drives expression immediately anterior to the MF. 


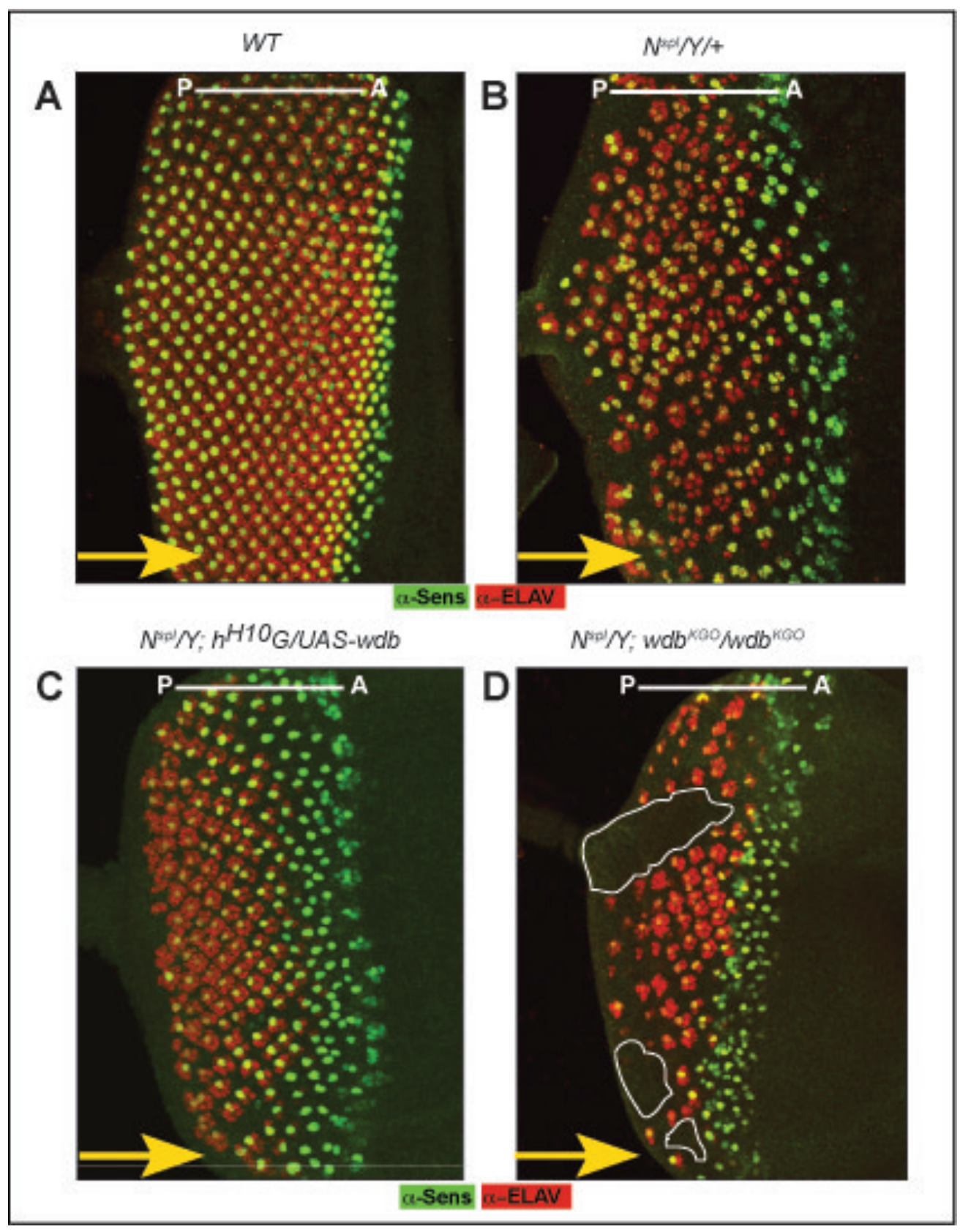

Fig. 4. Wdb effects on $\boldsymbol{N}^{\text {spl }}$ reflect R8 specification. The genotypes of the eye discs are wild type (A), $N^{\text {spl }} / Y(\mathrm{~B}), N^{\text {spl }} / Y ; h^{H 10}$ Gal4/UAS-wdb (C) and $N^{\text {spl }} Y ; w d b^{K G 02977} / w d b^{K G 02977}$ (D). Solid white lines denote posterior-anterior axis. Yellow arrows indicate direction of MF progression. In panel $D$ the encircled areas denote regions completely devoid of photoreceptors. 


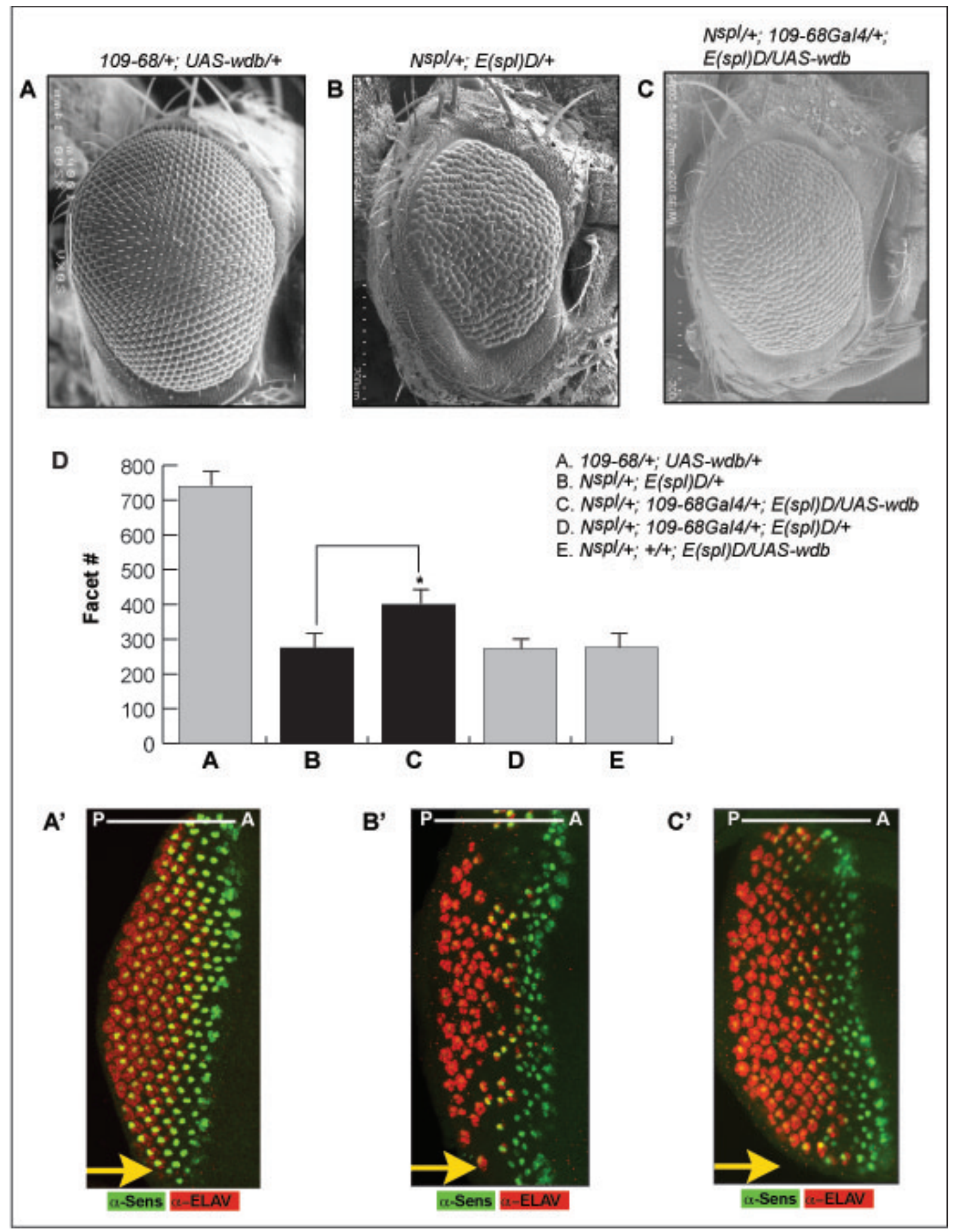


Fig. 5. Increased wdb dosage rescues the rough and reduced eye of $\boldsymbol{N}^{s p l} /+; E(s p l) D /+$ flies. (A-C) Adult eye phenotypes. The genotypes are 109-68/+; UAS-wdb (A), $N^{s p l} /+; E(s p l) D /+(\mathrm{B})$ and $N^{\text {spl } /+ \text {; }}$ 109-68/+; $E(s p l) D / U A S-w d b(C)$. The anterior of the eye is to the right. Magnification is 200X. (D) Quantitative analysis of the rescue determined by facet count in $\geq 10$ flies of each genotype. The relevant genotypes are indicated. Control genotypes are grey bars. The solid line denotes genotypes compared. Asterisk denotes $P$ value $<0.01$. ( $\left.A^{\prime}, B^{\prime}, C^{\prime}\right)$ Eye discs were immunostained with $\alpha$-Sens and $\alpha$-Elav. Genotypes of discs (A', B', C') correspond to those in panels (A, B, C) respectively. Solid white lines denote posterior-anterior axis. Yellow arrows denote direction of MF progression. 


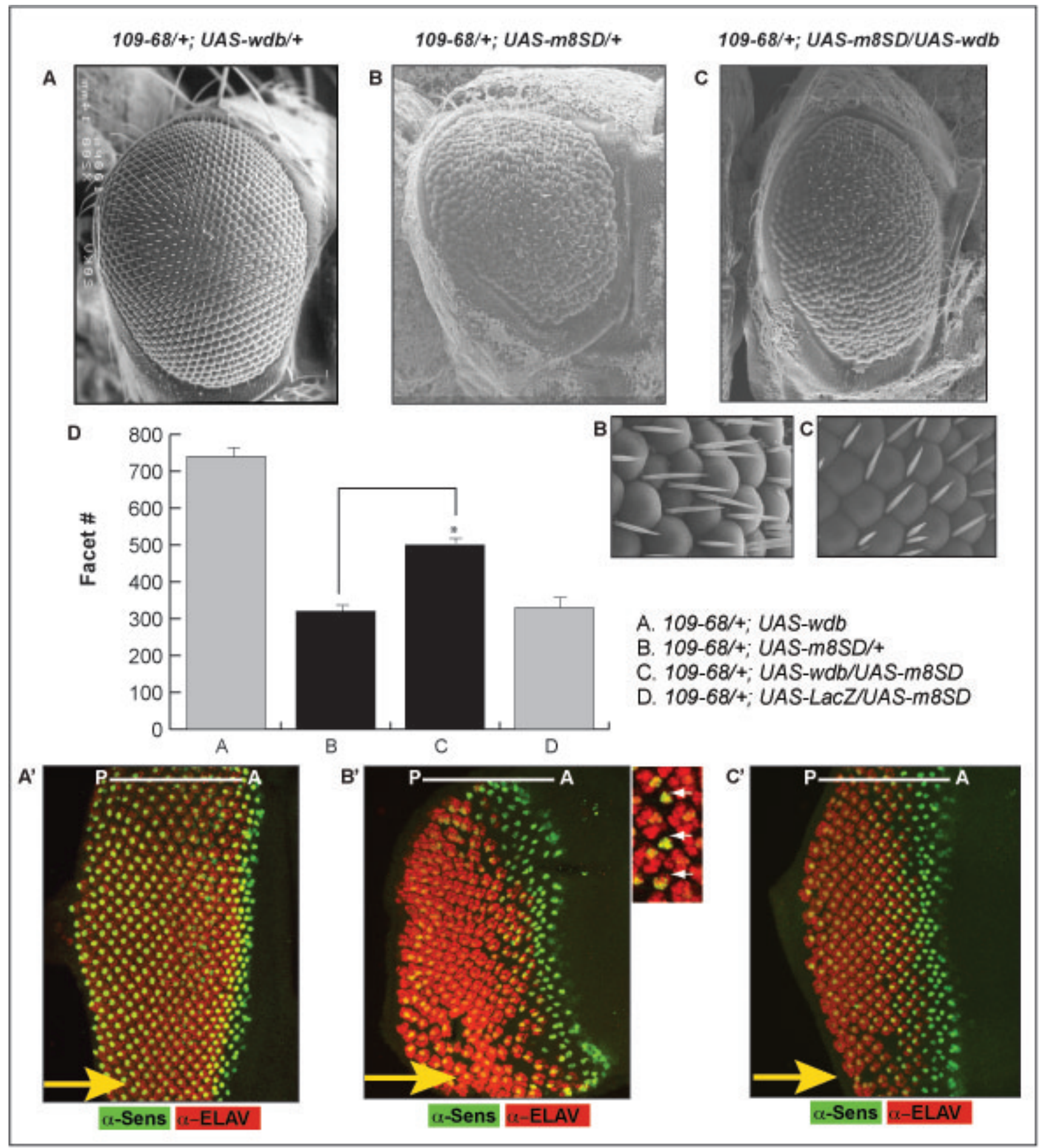


Fig. 6. Increased wdb dosage rescues the dominant retinal defects of ectopic M8SD.

(A-C) Adult eye phenotypes. The genotypes are 109-68/+; UAS-wdb (A), 109-68/+; UAS-m8SD/+ (B) and 109-68/+; UAS-m8SD/UAS-wdb (C). The anterior of the eye is to the right. Magnification is 200X. (B, C) inset magnification is $1000 X$ (D) Quantitative analysis of the rescue determined by facet count in $\geq 10$ flies of each genotype. The relevant genotypes are indicated. Control genotypes are grey bars. The solid line denotes compared genotypes. Asterisk denotes $P$ value $<0.01$. ( $\left.A^{\prime}, B^{\prime}, C^{\prime}\right)$ Eye discs were immunostained with $\alpha$-Sens and $\alpha$-Elav. Genotypes of discs (A', B', C') correspond to those in panels $(A, B, C)$ respectively. (B') white arrows in inset denote Sens+ cells (R8 photoreceptors) that lack Elav+ cell clusters (secondary photoreceptors). Solid white lines denote posterior-anterior axis. Yellow arrows denote direction of MF progression. 


\section{Chapter 4}

Rescue of Drosophila CK2 $\alpha$ mutants with temperature-sensitive alleles: A resource for analysis of CK2 functions in development. 


\section{Abstract:}

Protein kinase CK2 plays roles in diverse developmental programs, in addition to cell autonomous functions such as cell cycle progression and checkpoint control. Consequently, the complete loss of CK2 activity elicits early lethality, and the centromeric linkage of the CK2 $\alpha$ gene, which encodes the catalytic subunit of this protein kinase, has precluded analysis via mitotic clones. We have now rescued the lethality of CK2 mutants by the expression of putative $C K 2 \alpha$-ts alleles, which were previously generated using a yeast complementation assay. One of these is a variant that replaces $\mathrm{Gly}^{89}$ with Asp, a substitution that localizes to a critical loop in the vicinity of the active site. Expression of $C K 2 \alpha-G^{89} D\left(C K 2-G^{89} D\right)$ under control of the ubiquitous tubulin promoter rescues the pupal lethality of a hypomorphic allele $C K 2 \alpha^{M B 00477}$. We first demonstrate that $C K 2^{M B 00477}$ pharate adults exhibit rough and reduced eyes, due to the aberrant specification of the 'founding' R8 cells. Importantly, eye discs display 'twinned' R8's, a hallmark of loss of Notch pathway activity, in addition to defects in the morphogenetic furrow (MF). All of these defects are fully rescued by $C K 2-G^{89} D$ at the permissive temperature of $24^{\circ} \mathrm{C}$. At the non-permissive temperature of $32^{\circ} \mathrm{C}$, in contrast, $C K 2-G^{89} D$ appears to lose activity. Specifically, its ability to rescue $C K 2^{M B 00477}$ homozygotes is lost, and when temperature shifts are introduced later in development, ectopic bristle defects manifest. These defects are also characteristic of Notch loss of function. These studies provide a direct role for CK2 in eye and bristle development, and indicate that CK2$G^{89} D$ is a bona fide ts-allele. Given that analyses of the roles of CK2 in other developmental programs have necessitated reverse-genetic approaches, which are not always facile, flies rescued by a CK2-ts allele should be a resource of general utility to the Drosophila community. 


\section{Introduction}

The highly conserved and ubiquitous Ser/Thr kinase CK2 is involved in wide range of cellular functions that encompass cell cycle progression, transcription, translation and cell signaling (Hanna et al., 1995; Litchfield, 2003; Meggio and Pinna, 2003; ole-MoiYoi, 1995; Pepperkok et al., 1994). In addition to these cell autonomous functions, CK2 also plays roles in animal development. For example, the developmental roles of proteins such as Antennapedia, Engrailed, Odd-skipped and Ultrabithorax require phosphorylation by CK2 (Bourbon et al., 1995; Goldstein et al., 2005; Jaffe et al., 1997; Taghli-Lamallem et al., 2008). Moreover, recent studies have described roles for this kinase in Wingless/Wnt and Notch signaling pathways (Bose et al., 2006; Dominguez et al., 2004; Karandikar et al., 2004; Seldin et al., 2005).

Direct analysis of the role of CK2 in animal development has been less forthcoming because CK2 is required for cell cycle progression (Hanna et al., 1995; Rethinaswamy et al., 1998) and is essential for viability in yeast, mouse and Drosophila (Buchou et al., 2003; Lin et al., 2002; Lou et al., 2008; Padmanabha et al., 1990; Seldin et al., 2008). Null mutants of CK2 exhibit cell autonomous defects that lead to lethality, which in metazoan animals manifests early in development. Both in mouse and Drosophila, homozygous null mutants of CK2 are lethal (Buchou et al., 2003; Lin et al., 2002; Lou et al., 2008; Seldin et al., 2008). Therefore, in vivo studies of CK2 have focused on the identification of its targets based upon the presence of consensus sites for phosphorylation, followed by the expression of variants that are nonphosphorylatable or mimic the constitutively phosphorylated state to assess the relevance of these interactions (Bourbon et al., 1995; Jaffe et al., 1997; Karandikar et al., 2004; TaghliLamallem et al., 2008).

In addition to the analysis of CK2 targets, a direct assessment of the role of CK2 in vivo has been approached by conditional inactivation of CK2 such as by RNA interference 
(RNAi). The targeted expression of RNAi constructs would allow stage specific analysis of the roles of CK2 in development, bypassing early embryonic requirements for this enzyme. Studies to generate mitotic clones have been precluded by centromeric linkage of $C K 2 \alpha$. Given the availability of diverse molecular tools for spatial and temporal inactivation of genes, Drosophila has emerged as an ideal model system for such analysis. Moreover, human diseases and mammalian development are increasingly been modeled in this facile genetic model (Bier, 2005; Jackson et al., 2002; Joutel et al., 1996; Shulman and Feany, 2003).

In Chapter 2, we employed the commonly used Gal4-UAS (Brand and Perrimon, 1993) mediated expression of RNAi constructs (Lee and Carthew, 2003) for conditional inactivation of CK2. These studies have demonstrated a role in Notch signaling. While the RNAi approach has enabled analyses of CK2 function during patterning of R8 photoreceptors and SOP's, one of the problems encountered was a hysteretic delay in the onset of the dominant neural defects. Specifically, in the eye, R8 patterning defects were elicited only upon expression of CK2-RNAi constructs in a broad expression domain anterior to the morphogenetic furrow (MF) with eyeless (ey) Gal4 (Bose et al., 2006). On the other hand, expression of CK2-RNAi in the MF during R8 selection (with scaGal4), or immediately anterior to the MF during R8 cluster formation (with $h$ Gal4) did not elicit R8 defects (Bose et al., 2006). The possibility arose that expression with scaGal4 or $h$ Gal4 did not permit enough time to attenuate CK2 levels. This refractory period could therefore reflect the time required for turnover of preformed (endogenous) CK2 protein to a level that becomes rate limiting for lateral inhibition. As a result, this necessitated the expression of CK2-RNAi constructs prior to the onset of retinal patterning. Lateral inhibition is also involved in the specification of the R2/5, R3/4, R1/6 and R7 cell fates (Cagan and Ready, 1989a; Cooper and Bray, 1999a; Cooper and Bray, 2000; Fanto and Mlodzik, 1999; Tomlinson and Struhl, 2001), but these could not be analyzed for the aforementioned reason. 
The availability of conditional alleles, i.e., those that are temperature sensitive (ts) for enzymatic activity, would circumvent this problem with the RNAi approach. ts-alleles can often display a rapid onset of enzyme inactivation, and thus enable a more precise modulation of CK2 functions. The other advantage of ts-alleles is that they should enable a systematic analysis of CK2 functions during specification of secondary photoreceptors, at later steps in retinal patterning (see above).

Previous studies in our laboratory led to the generation of a number of putative $C K 2 \alpha$ ts alleles (Kuntamalla et al., 2009). These are not 'alleles' in the strictest sense, but this designation is used as a generic descriptor. The alleles were generated by site-specific replacements of residues, which were identified based on extant ts-alleles of yeast-CK2 and by molecular modeling of Drosophila CK2 $\alpha$ using the atomic coordinates of human CK2 (Kuntamalla et al., 2009). The two alleles used in these studies are $C K 2-G^{89} D$ and $C K 2-A^{177} T$. The $G^{89} D$ substitution is localized to a loop that connects the $\mathrm{N}$ - and C-terminal domains of CK2 $\alpha$ (Fig. 1A). It is expected that the replacement of glycine with aspartate may cause steric hinderance of the architecture of this loop. On the other hand, the $A^{177} T$ substitution is located in a hydrophobic pocket in the activation segment (Fig. 1B), thereby leading to a destabilization of phosphotranferase activity. Therefore, these substitutions may affect kinase functions without gross structural abnormalities.

These alleles have been tested for putative ts-behavior based on their ability to rescue a yeast strain lacking endogenous CK2 (Kuntamalla et al., 2009). Analysis with these rescued yeast strains revealed that the minimum permissive temperature (PT) and maximum nonpermissive temperature (NPT) for $C K 2-G^{89} \mathrm{D}$ are $35^{\circ} \mathrm{C}$ and $37^{\circ} \mathrm{C}$, respectively. Consistent with its presence in the activation segment, $C K 2-A^{177} T$ is more sensitive, and its PT and NPT are $32^{\circ} \mathrm{C}$ and $35^{\circ} \mathrm{C}$, respectively (Kuntamalla et al., 2009). These studies indicated that these two Drosophila CK2-ts alleles have distinct NPT's. 
We, therefore, decided to use these alleles to rescue null or hypomorphic alleles of Drosophila CK2. The studies of this chapter demonstrate rescue of lethality of flies lacking CK2. In addition, direct roles for CK2 in R8 and SOP selection are now evidenced. Given multiple developmental roles of this enzyme, this molecular modeling and reverse genetic approach have resulted in a resource that should be of general interest to the Drosophila community.

\section{Results and Discussion:}

\section{Mutations in Drosophila CK2 $\alpha$ :}

The first allele of $C K 2 \alpha$ was identified based on its ability to dominantly perturb the circadian clock. This allele, called Timekeeper (Tik, Fig. 2A), is a CK2 dominant-negative $(D N)$ that encodes a catalytically dead subunit (Lin et al., 2002; Rasmussen et al., 2005). Unlike its clock defect, Tik/+ flies display no overt defects in the eye or the macrochaetes. However, Tik homozygotes die at the first larval instar (Fig. 2B) precluding analysis of CK2 at later stages of development. A second allele, called $\mathrm{Tik}^{R}$, was identified as a spontaneous revertant whose clock defects are more muted. $T_{i k}{ }^{R}$ harbors additional mutations, which appear to impair the overall structure of the catalytic subunit (Kunttas-Tatli et al., 2009). Consequently, the TikR protein does not interact with the regulatory CK2 $\beta$ subunit, an interaction that is essential for the formation of the $\alpha_{2} \beta_{2}$ holoenzyme (Bidwai et al., 1992; Glover et al., 1983). This defect is not associated with Tik. Together these studies indicate that the DN-behavior of Tik reflects its incorporation into and poisoning of the endogenous holoenzyme. In contrast, $\operatorname{Tik}^{R}$ might be closer to a protein/genetic null. Accordingly, Tik $^{R}$ is also lethal when homozygous and the effective lethal phase occurs at the first larval instar (Fig. 2B). 
More recently a third allele of $C K 2 \alpha$ was identified. This allele called $C K 2^{M B 00477}$ involves the insertion of a minos element between two alternative, non-coding exons of the CK2 $\alpha$ gene (Fig. 2A). CK2 $2^{M B 00477} /+$ flies are viable and fertile and do not display developmental defects, but is pupal lethal when homozygous. Lethality occcurs relatively late during pupariation and analysis of pharate adults indicates that flies homozygous for $C K 2^{M B 00477}$ display rough eyes and ectopic MC's (Fig. 2B), previously described using the RNAi approach (Chapter 2, see above). Similar phenotypes are seen in flies that are transheterozygous for CK2 $2^{\text {MB00477 }}$ and either Tik or Tik ${ }^{R}$.

\section{The eye phenotype of $C K 2^{M B 00477}$ pharate adults:}

We first analyzed the eye defects of $C K 2^{M B 00477} / C K 2^{M B 00477}$ and $C K 2^{M B 00477} / T$ ik pharate adults. Both genotypes exhibit a reduced and rough eye phenotype (Fig. 3A, B) characterized by a loss in ommatidial phasing and the misassignation of interommatidial bristles (Fig. 3A', B'). In addition, a number of ectopic and fused ommatidia can be observed (Fig. 3B', yellow asterisk). None of these defects are seen in $C K 2^{M B 00477} /+$ or Tik/+ flies (data not shown, and see below). All of these aberrations have been observed upon expression of CK2-RNAi in the developing retina (Chapter 2, see above), although with reduced severity.

We next analyzed CK2 ${ }^{M B 00477}$ homozygous eye discs for the expression of Sens (R8specific marker) and Elav (pan-neural marker). While CK2 $2^{M B 00477} /+$ discs (Fig. 3C) display single, evenly spaced R8's, CK2 $2^{M B 00477} / C K 2^{M B 00477}$ discs exhibit ectopic and twinned R8's (Fig. 3A"). In addition, these excess R8's continue to maintain uniform levels of Sens expression, indicating that they are adopting the differentiated R8 fate efficiently. Consistent with this interpretation, these ectopic R8's initiate the process of secondary photoreceptor recruitment (Fig. 3A", inset). The lack of a normal complement of secondary (R1-R7) photoreceptors may reflect competition between adjacent R8's for a limited pool of retinal progenitor cells. In addition, we observe novel retinal defects (overlapping photoreceptor clusters) in $C K 2^{M B 00477}$ 
homozygous eye disc (Fig. 3A", dotted circle). This phenotype may be due to defects in the initiation of the morphogenetic furrow (MF, see below). Nevertheless, the R8 defects are consistent with our previous findings using CK2-RNAi (Chapter 2). In addition to the eye, $C K 2^{M B 00477} / C K 2^{M B 00477}$ adults also display ectopic MC's (data not shown) akin to those observed with CK2-RNAi.

\section{Compromised CK2 activity elicits defects in the morphogenetic furrow:}

During studies of R8 patterning defects in $C K 2^{M B 00477} / C K 2^{M B 00477}$ discs, we observed a region where photoreceptors appeared to be densely clustered (Fig. 3A"). In a Z-scan across the eye disc, a monolayer neuro-epithelium (Fig. 4A), this region of the eye disc displays Sens+ and Elav+ photoreceptors at two focal planes (Fig. 4A', A", see arrow). Consequently, images taken at these two planes uncover a gap in the center of the eye disc, that appears to lack any differentiated R8's or secondary R-cells. Imaging at a higher focal plane (Fig. 4A) indicates the presence of relevant R-cells in this 'gap', whose staining with Sens and Elav indicates that they are differentiated photoreceptors (Fig. 4A"). This defect appears consistently in homozygous $C K 2^{M B 00477}$ eye discs and the region is invariably located towards the posterior of the eye disc and spans the equator. Moreover, a closer examination reveals that Sens+ and Elav+ cells posterior to this region display mispatterning.

During eye development Notch is required at multiple steps, including the initiation of retinal histogenesis to the specification of successive photoreceptor cell fates (Cagan and Ready, 1989a; Kumar and Moses, 2001; Lee and Treisman, 2002). At present the mechanism for these defects is unclear, but may result from the induction of ectopic furrows, previously observed by others (Chanut and Heberlein, 1995). Our earlier studies with CK2RNAi have indicated a role for CK2 in MF initiation. The initiation of the MF is divided into two distinct phases (Kumar and Moses, 2001). The first is the initiation of the furrow at the posterior margin of the eye disc (Fig. 4B, furrow birth, see red arc) and the second phase 
termed 'furrow reincarnation' occurs at the D/V margins of the eye disc (Fig. 4B, furrow reincarnation, see orange arc). Studies indicate that these two phases are temporally and genetically seperable (Kumar and Moses, 2001). Furrow reincarnation is under the control of Egfr, Notch and Dpp signaling (Fig. 4B). Loss of either Egfr or Notch signaling leads to furrow defects at the lateral margins, but do not seem to affect initiation. Consequently, the furrow progresses only from the posterior margin of the eye disc as an arc'. A similar MF defect is seen upon targeted expression of a CK2-RNAi construct early during eye development (Fig. 4C, C'). This defect closely mimicks that reported with loss of Notch, Delta and E(spl) function (Kumar and Moses, 2001). Of interest, the only $\mathrm{E}(\mathrm{spl})$ member implicated in MF initiation is M8, a known target of CK2. Therefore, the possibility arises that CK2 may play a role in MF initiation, as well. Further analysis employing mutants of Egfr, $N, C K 2$ and $d p p$ would be required for defining the role of CK2 in proper formation of a uniform and linear MF.

\section{Rescue by putative ts-alleles of Drosophila CK2 $\alpha$ :}

We next sought to employ the putative CK2-ts alleles to rescue homozygous null mutants of Drosophila CK2 $\alpha$. We first used the Gal4-UAS approach, in which the CK2-ts alleles were expressed with the ubiquitous act5C-Gal4 driver (Fig. 5). However, the Gal4UAS system was found to be unsuitable as no rescue of Tik, TikR, or CK2 $2^{\text {MB00477 }}$ homozygous flies was evidenced (data not shown). We reasoned that act5C-Gal4 driver may not have expressivity at a level sufficient for rescue. We therefore tested the putative CK2-ts alleles under the contol of the actin promoter. In the case of $C K 2 \beta$, this promoter has been reported to be sufficient to rescue null alleles with site-specific variants of CK2 $\beta$ (Jauch et al., 2002). For reasons that remain unclear, the actin promoter was found to also be unsuitable (data not shown). The lack of rescue does not reflect an inactive protein, because wild type CK2 $\alpha$ under control of the actin promoter has since been found to be insufficient, whereas the tubulin promoter appears to be sufficient for rescue (Andreas Jenny, personal 
communication). We therefore decided to test the ubiquitous tubulin promoter (Fig. 5) to drive expression of the $C K 2-G^{89} D$ and $C K 2-A^{177} T$ alleles.

\section{Rescue of $C K 2^{M B 00477}$ pharate adults by tub-CK2-G ${ }^{89} D$ :}

We first employed the $C K 2-G^{89} D$ because it is more stable (in yeast), as compared to CK2-A ${ }^{177} T$ (see above). Instead of using Tik or TikR, we focused on the hypomorphic allele CK2 $2^{M B 00477}$. A schematic showing the rescue strategy is outlined in Fig. 6A. Females of the genotype tub-CK2-G ${ }^{89} D / t u b-C K 2-G^{89} D ; T m 6 B, T b /+$ were crossed to $C K 2^{M B 00477} / T m 3, S b^{1}$ males. The $\mathrm{F} 1$ progeny $\left(\mathrm{Sb}^{+}, \mathrm{Tb}\right)$ males and females were next mated and viable progeny were identified for the absence of $T m 6 B, T b$ balancer chromosome (Fig. 6A). In this cross $\mathrm{Tb}^{+}$ progeny would be homozygous for the $C K 2^{M B 00477}$ allele, and the presence of $t u b-C K 2-G^{89} D$ would manifest as a red eye. From this cross, we find that all $\mathrm{Tb}^{+}$adults that eclose have the tub-CK2-G ${ }^{89} D$ allele. In addition, the minos insertion is marked by GFP (see Fig. 2) expressed under the control of an eye specific (eyeless) enhancer (Metaxakis et al., 2005). We find that rescued flies express GFP with an intensity that is twice that of $C K 2^{M B 00477} /+$ flies (data not shown).

We then sought to verify rescue, assuming that $24^{\circ} \mathrm{C}$ would be close to or at the PT. For this analysis we crossed tub-CK2-G ${ }^{89} D / Y ; C K 2^{M B 00477} / C K 2^{M B 00477}$ (rescued males) with $C K 2^{M B 00477} / T m 3, S b^{1}$ females. As expected, the $\mathrm{Sb}^{+}$males from this cross did not eclose, whereas females rescued by a single dose of $t u b-C K 2-G^{89} D$ survived to adulthood. Therefore, $t u b-C K 2-G^{89} D$ rescues as a single copy. Similar crosses were also conducted at $32^{\circ} \mathrm{C}$, and at this higher temperature tub-CK2-G ${ }^{89} \mathrm{D} /+; C K 2^{M B 00477} / C K 2^{M B 00477}$ females were pupal lethal. It would thus appear to be the case that CK2 with a substitution of Gly ${ }^{89}$ with Asp, indeed, elicits ts-behavior.

\section{Phenotype of rescued flies:}


Following rescue of lethality in $C K 2^{M B 00477} / C K 2^{M B 00477}$ flies by tub-CK2-G ${ }^{89} D$, we analyzed the eye and bristle phenotypes in these rescued flies. We find that expression of the $C K 2-G^{89} D$ allele rescues the rough and reduced eye (Fig. $\left.7 A\right)$. Importantly, these rescued flies display a highly organized adult eye (Fig. 7A') where facet and IOB patterning mimic those of wild type flies (not shown) or those in $C K 2^{M B 00477} /+$ flies (Fig. 7B).

We next sought to assess if a shift to the NPT would elicit eye/MC defects. Given that CK2-G ${ }^{89} \mathrm{D}$ is non-functional at $32^{\circ} \mathrm{C}$, we conducted a temperature shift regimen schematically shown in Fig. $7 \mathrm{C}$. In this regimen, animals were transfered to $32^{\circ} \mathrm{C}$ from $\mathrm{L} 3$ to the mid-pupal stage, as this would cover both R8 and SOP selection. We find that $\sim 10 \%$ of rescued animals maintained at $24^{\circ} \mathrm{C}$ display on average $4.2 \pm 0.2 \mathrm{MC}$ 's/scutellum, a number slightly higher than that in otherwise wild type flies (data not shown). This would indicate that even at this lower temperature $C K 2-G^{89} D$ may be slightly attenuated in activity. Both, the penetrance and the expressivity of the ectopic MC phenotype were enhanced when cultures were raised at higher temperatures; the effect at $29^{\circ} \mathrm{C}$ was more muted than that at $32^{\circ} \mathrm{C}$. These results essentially corroborate our previous findings on the role of CK2 in MC development. A similar analysis in the eye did not bear out (data not shown). The possibility remains that eye development is more refractory to fluctuations in CK2 levels or activity, as previously proposed by us (Bose et al., 2006). Moreover, specification of the R8's is crucial for eye development and the hexagonal patterning of the adult compound eye. Since perturbations in either process are likely to negatively impact vision and thus survival, as compared to the effects of a misassigned bristle, evolution may have selected for a greater threshold of CK2, a critical regulator of M8 activity.

Given the muted temperature sensitivity of $C K 2-G^{89} D$, shifts to $32^{\circ} \mathrm{C}$ at earlier stages of development, or the use of higher temperatures may be necessary to inactivate CK2 to a level that will compromise lateral inhibition and R8 selection. Towards this end, the $C K 2-A^{177} T$ 
allele (Fig. 1B), which exhibits greater ts-behavior (in yeast), has now been used to rescue $C K 2^{M B 00477} / C K 2^{M B 00477}$ flies (data not shown). Analysis of these flies is currently underway. Alternatively, CK2 ts-alleles could be analyzed in backgrounds heterozygous for CDC37, a dosage-sensitive chaperone for CK2. In yeast, CK2-ts alleles are hyper-sensitized by reduced dosage of $c d c 37$ (Bandhakavi et al., 2002). In Drosophila, a background heterozygous for $c d c 37$ may accentuate the ts-behavior of $C K 2-G^{89} D$ or may engender a more rapid inactivation at the NPT.

The virtually complete rescue of the retinal and bristle defects of homozygous $C K 2^{M B 00477}$ indicates that the associated lethality and the retinal and the bristle defects are specifically due to loss of CK2 activity. In addition, the pupal lethality of Tik/CK2 $2^{M B 0047}$ or the larval 1 lethality of Tik/Tik has also been rescued by $t u b-C K 2-G^{89} D$ (data not shown). As with $C K 2^{M B 00477}$, these flies are viable and fertile. However, analysis of R8 patterning defects in rescued Tik homozygotes is precluded by the presence of roughoid, a recessive allele that perturbs the eye, independent of CK2 (Fig. 7D). For studies in the eye, this recessive allele will have to be recombined away from Tik. These complete rescues indicate that the expression of the $C K 2-G^{89} D$ allele from the tubulin promoter is robust and comparable to the levels of expression from the endogenous CK2 promoter.

In summary, the results reported in this chapter have led to the characterization of a bona fide $C K 2$-ts allele, $C K 2 G^{89} D$ that is stable and functional at permissive temperature, rescues null and hypomorphic mutants of CK2 and exhibits temperature sensitivity. This CK2ts-allele should be a resource for in vivo studies of CK2 functions in other developmental contexts, and should thus be of general utility to the Drosophila community.

\section{Experimental Procedures}




\section{Generation of transgenic flies:}

The previously generated cDNAs encoding the ts variants of Drosophila CK2 $\alpha$ (CK2$A^{177} T$ ) was isolated and subcloned into Drosophila transformation vector pCaSpeR-act. The inserts were subcloned into the $\mathrm{BamH} 1$ site and clones with proper orientation of the insert with respect to the actin promoter were isolated with restriction mapping. For this, internal restriction site in $\mathrm{CK} 2 \alpha$ (Nrul) and the additional restriction sites in pCaSpeR-act (HindIII, Sall and Pstl) were employed. This initial screen was followed by sequencing of the clone for final confirmation of the orientation of the insert in pCaSpeR-act-Bam. In the Drosophila transformation vector pCaSper-tub, the ts-variants of Drosophila CK2 $\alpha(C K 2-A 177 T$, CK2G89D) were directionally subcloned into the BamHI and Xho1 sites. The transgenes are thus downstream of the ubiquitous act5C or tubulin promoter. Multiple transgenic lines with the ts

alleles of Drosophila CK2 $\alpha$ were generated by germline transformation of $w^{1118}$ embryos. Red eye colored $\left(w^{+}\right)$progeny were identified and the location of the transgene insertions determined by crosses with flies harboring balancer chromosomes containing dominant visible markers.

\section{Fly crosses and phenotype analysis:}

Flies were raised at $24^{\circ} \mathrm{C}$ on standard yeast-glucose medium. All crosses for the generation of rescue stock were performed at $24^{\circ} \mathrm{C}$. Temperature sensitive analysis of the rescue stocks was conducted at $24^{\circ} \mathrm{C}, 29^{\circ} \mathrm{C}$ and $32^{\circ} \mathrm{C}$. In case of pupal lethal flies, pharate adults were dissected for analysis of adult eye and bristle phenotypes. Adult fly heads were passed through a graded alcohol series for 24 hours each (25-50-75-absolute). Finally, heads were passed through Hexamethyldisalizane, and mounted on EM stubs using carbon tape (Ted Pella). Fly heads were dried for 24 hours, sputter coated with gold, and examined with a JEOL-6400 scanning electron microscope at an accelerating voltage of $20 \mathrm{kV}$. Images were acquired, processed with Adobe Photoshop and collated in Adobe Illustrator. For bristle 
phenotypes, newly eclosed adults or dissected pharate adults were photographed using a Nikon digital camera attached to a Leica MZ16 stereomicroscope. Immunohistochemical analysis of eye discs were performed as described in Chapter 3. 


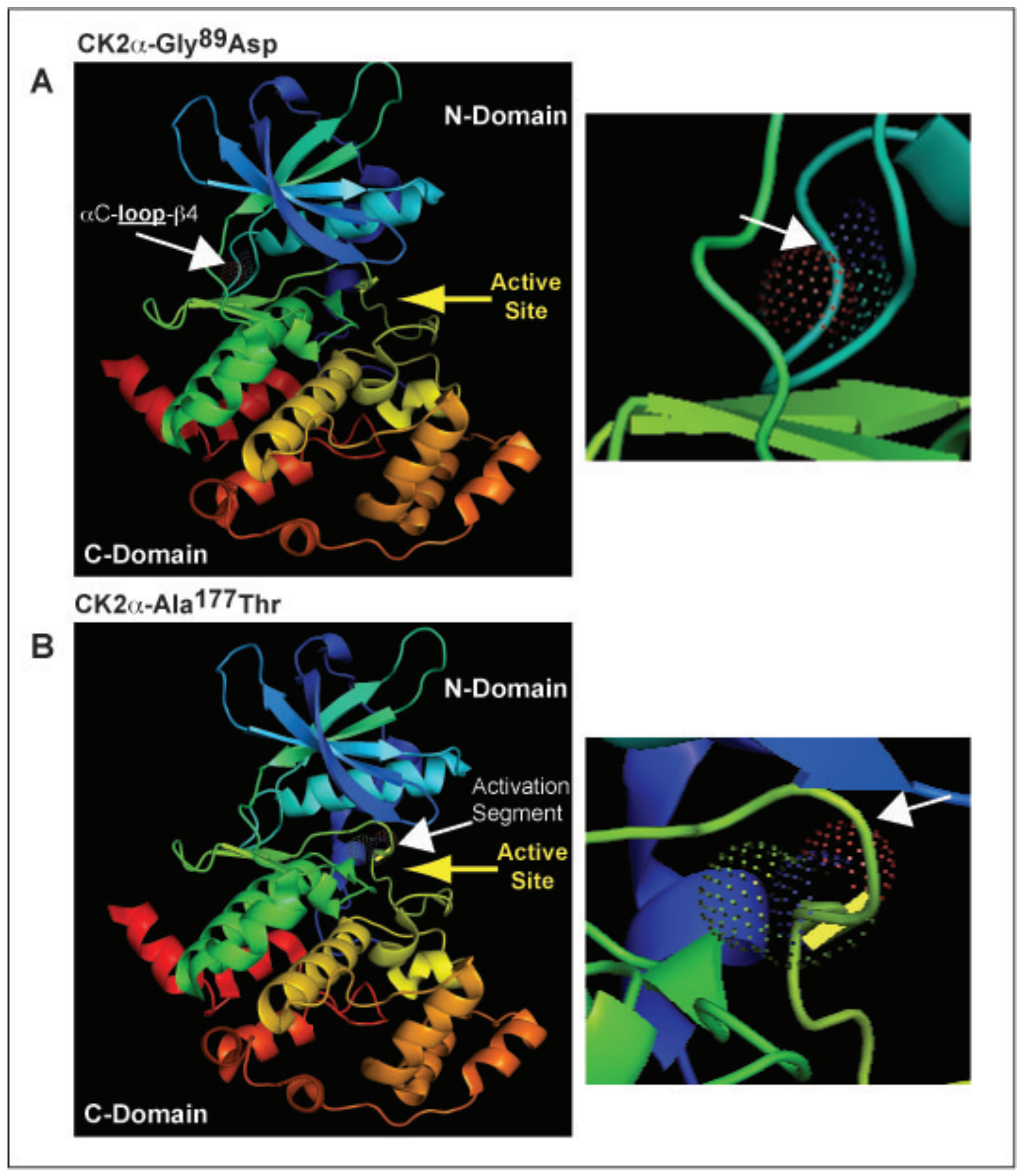

Fig. 1. Molecular modeling of the TS variants of Drosophila CK2 $\alpha$. (A) The localization of the $G^{89} D$ substitution. (B) The localization of the $A^{177} T$ substitution. The structural simulation was done using the PyMol molecular modeling software. The crystal co-ordinates of human CK2 $\alpha$ (PDB\# 1LP4) were used as the basis for model generation. 


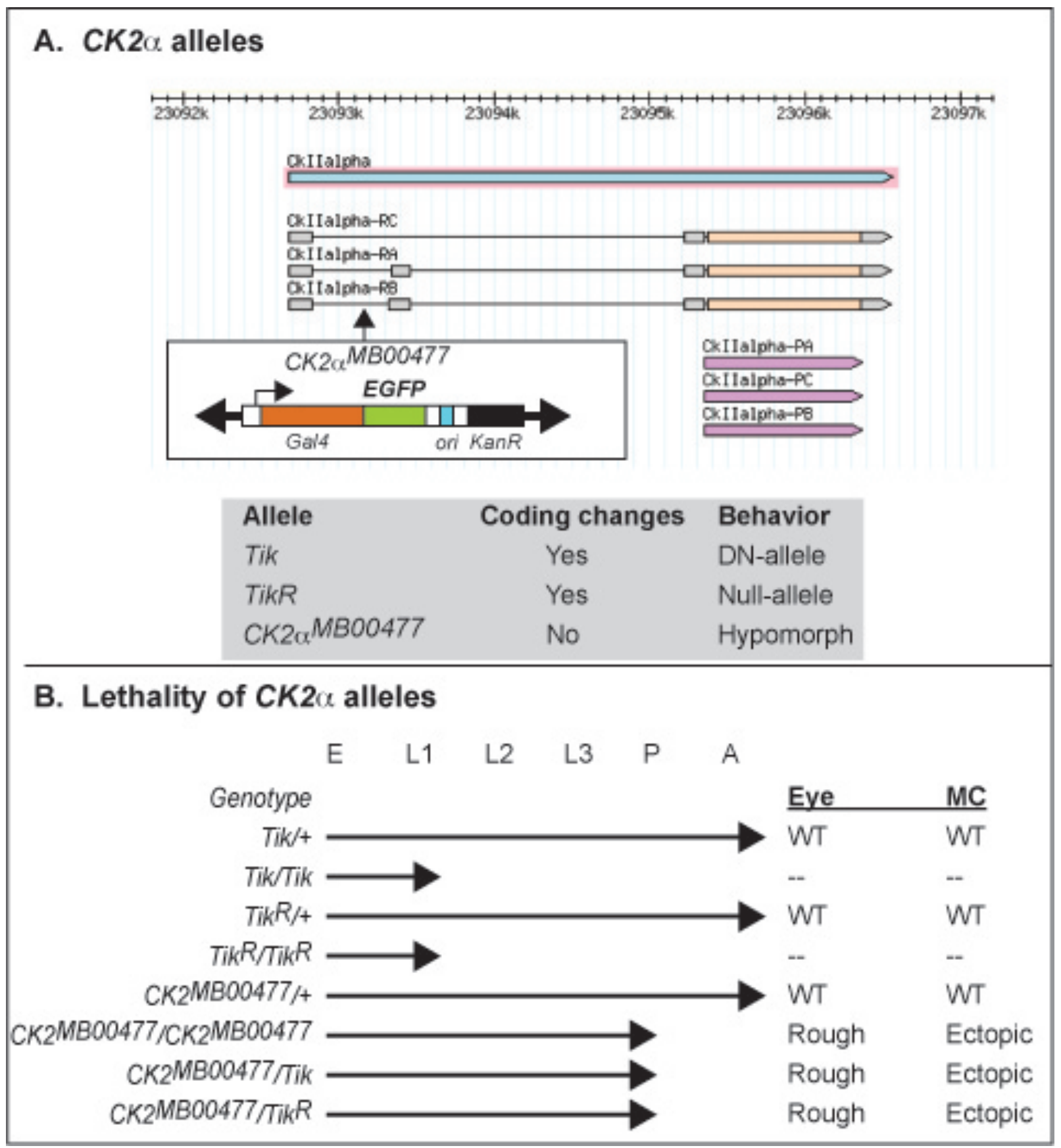

Fig. 2. A schematic representation of of the Drosophila CK2 $\alpha$ mutants and their developmental progression. (A) The $C K 2 \alpha$ alleles. (B) Schematic representation of the lethality of $C K 2 \alpha$ mutants. Eye and macrochaete defects for each genotype are denoted on the right side. 


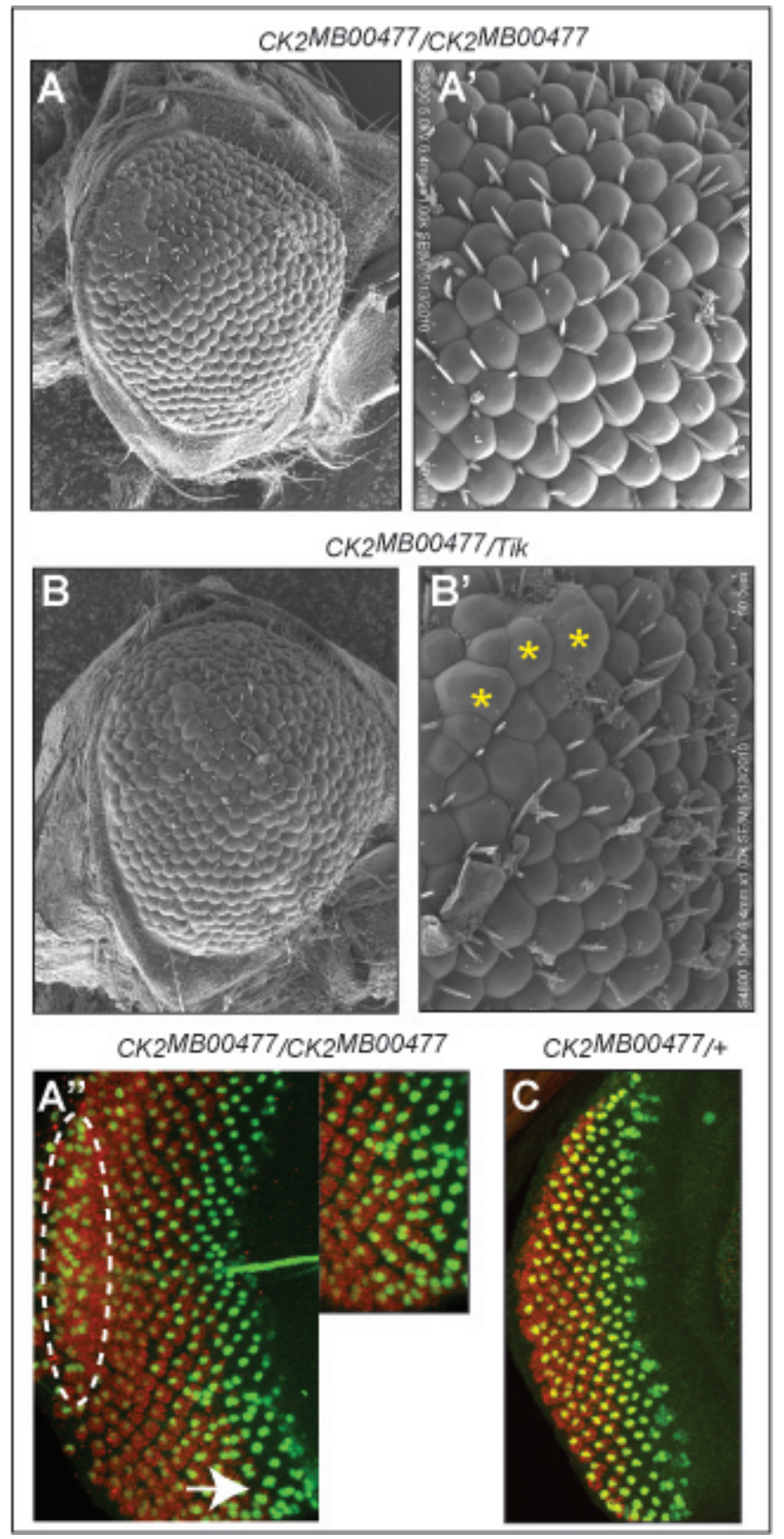


Fig. 3. Phenotypic analysis of homozygous $C K 2^{M B 00477} / C K 2^{M B 00477}$ flies. (A-B') Adult eye phenotypes. The genotypes are (A, A', A") $C K 2^{M B 00477} / C K 2^{M B 00477}$. (B, B') $C K 2^{M B 00477} /$ Tik (C) $C K 2^{M B 00477} /+$. Magnifications (A, B) 200X and (A', B') 1000X. (A", C) Eye discs are stained with $\alpha-$ Sens (green) and Elav (red). The white arrow indicates ectopic R8's. The white dotted circle denotes the densely clustered photoreceptors. 


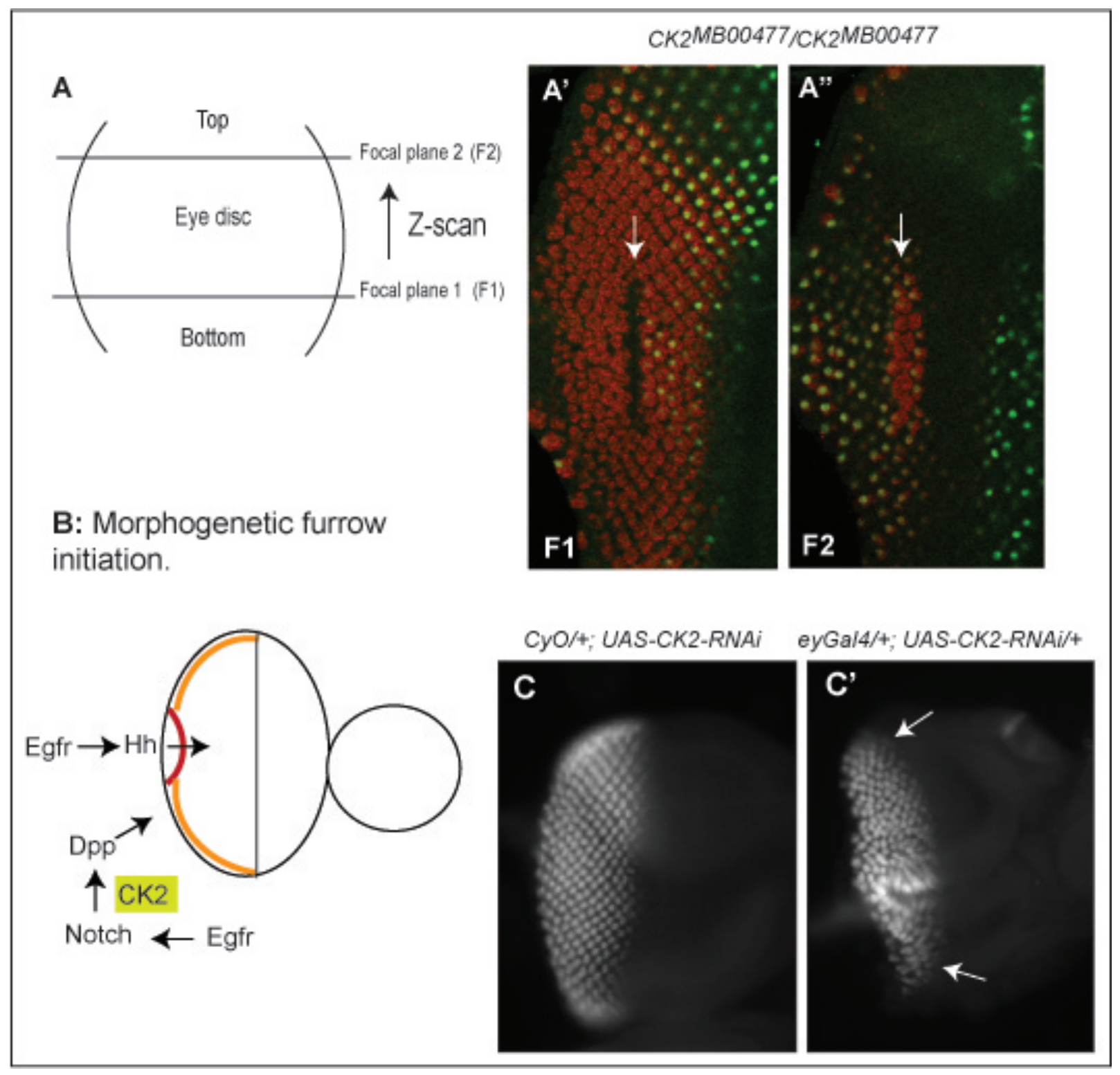

Fig. 4. Compromised CK2 activity elicits morphogenetic furrow defects. (A) Schematic of the Zscan, indicating the two focal planes. (A, A') Eye disc of $C K 2^{M B 00477} / C K 2^{M B 00477}$, arrow indicates the position of the scar. The focal planes are indicated, eye discs stained with $\alpha$-Sens and Elav. (B) A schematic representation of MF initiation. (C, C') Eye discs of CyO/+; UAS-CK2-RNAi/+ and ey-Ga/4/+; UAS-CK2-RNAi/+ stained with Elav. 


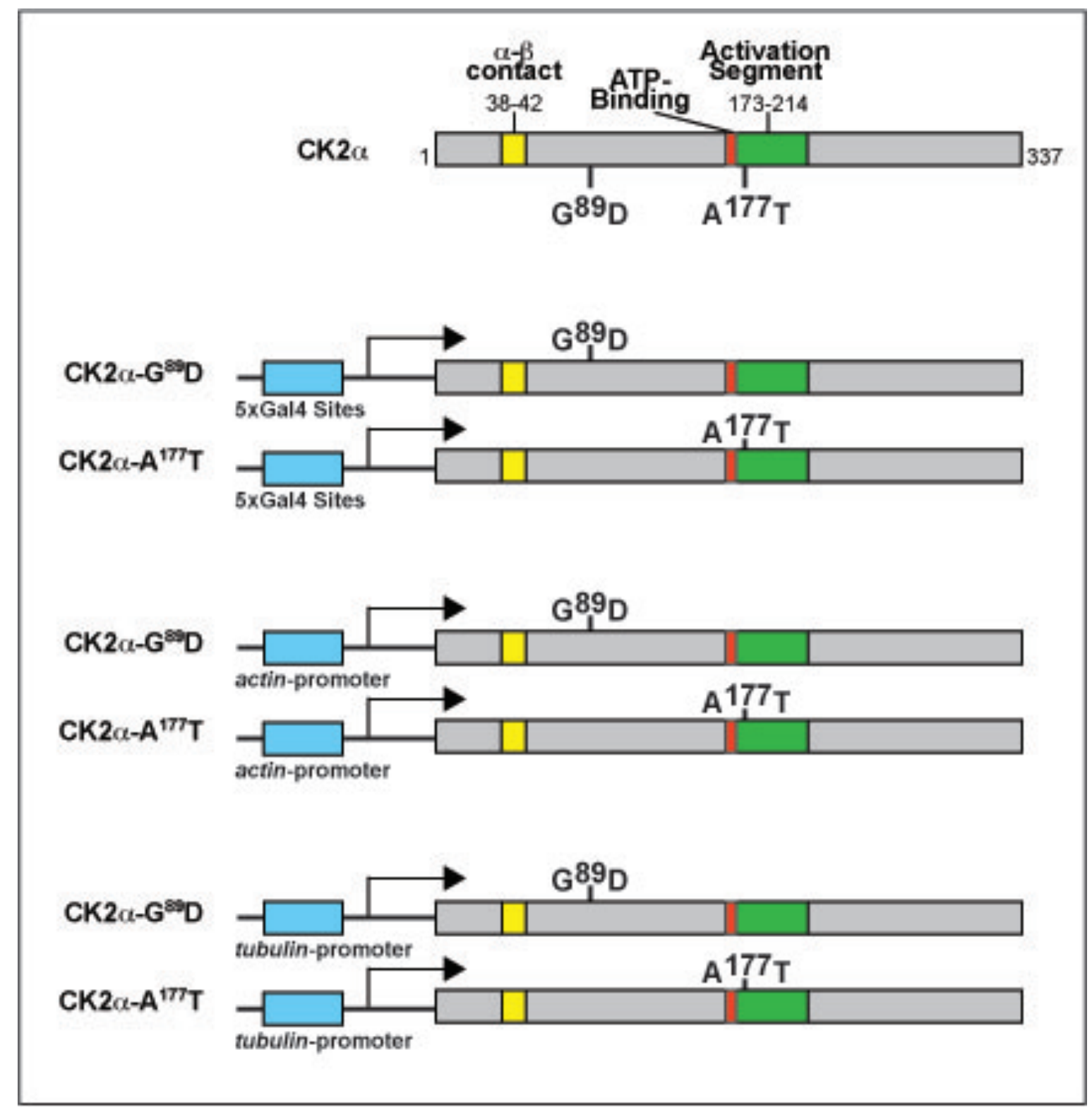

Fig. 5. A schematic representation of the constructs employed for rescue of homozygous null mutants of Drosophila CK2 $\alpha$. The localization of the substitutions with respect to the different domains of $\mathrm{CK} 2 \alpha$ is denoted. The activation segment, ATP binding pocket and the contact region between CK2 $\alpha$ and CK2 $\beta$ are indicated. 


\section{A. Rescue by CK2 $\alpha$ alleles}

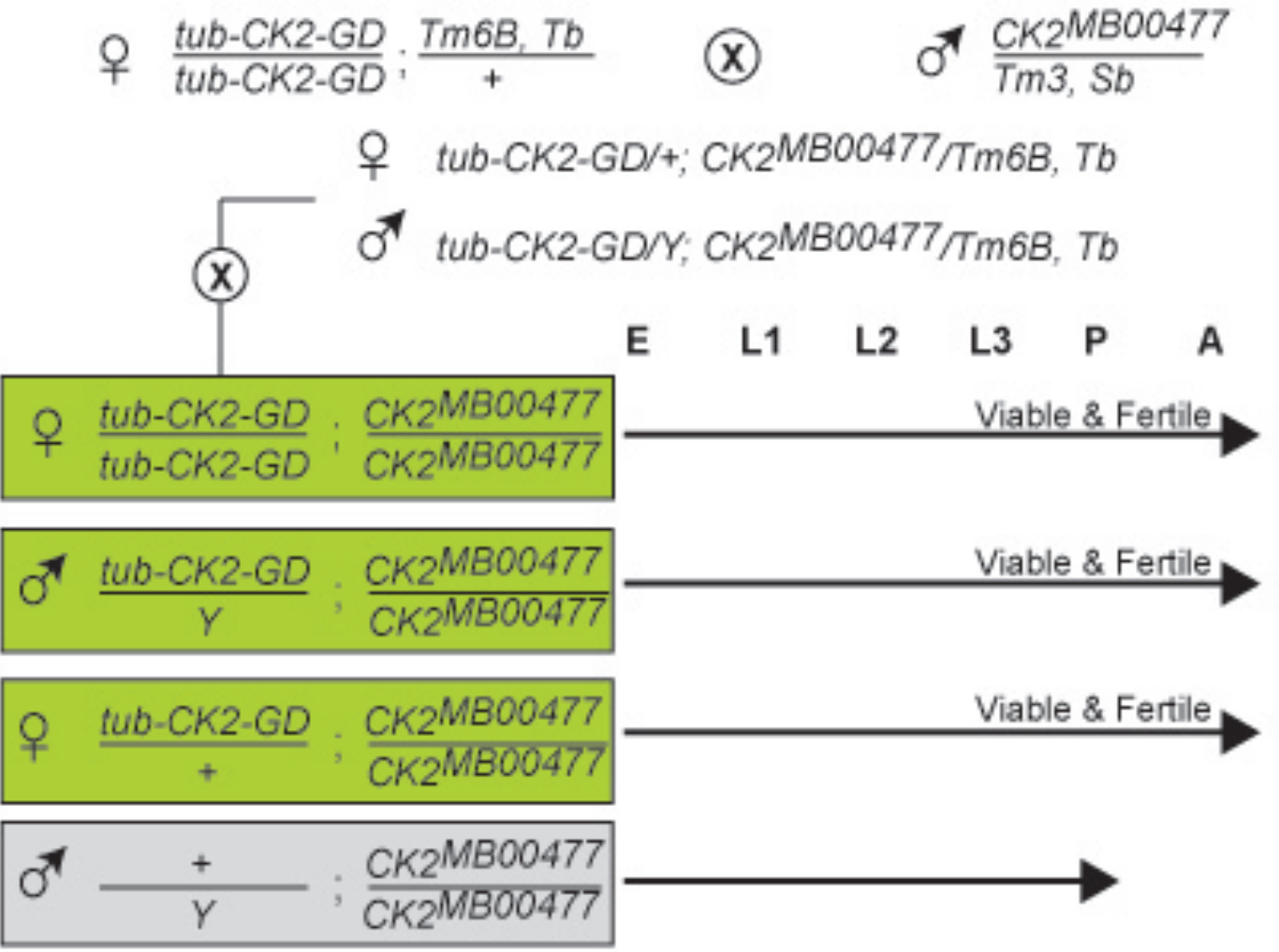

B. Rescue at 24-vs-320 C

ot $\frac{\text { tub-CK2-GD }}{Y} ; \frac{C K 2^{M B 00477}}{C K 2^{M B 00477}} \otimes$ 우 $\frac{C K 2 M B 00477}{T m 3, S b}$

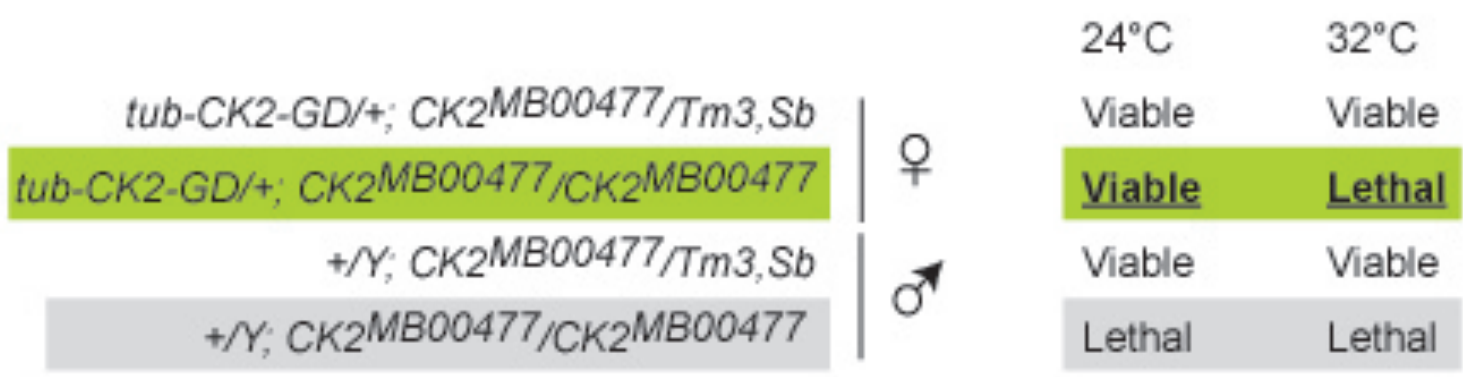

Fig. 6. The scheme for generation and verification of rescue stock. (A) Rescue by $C K 2 \alpha$ ts-allele.

(B) The rescue at $24^{\circ} \mathrm{C}-\mathrm{vs}-32^{\circ} \mathrm{C}$. The genotype of rescued male and female flies indicated by green box. The genotype of pupal lethal male indicated by grey box. 


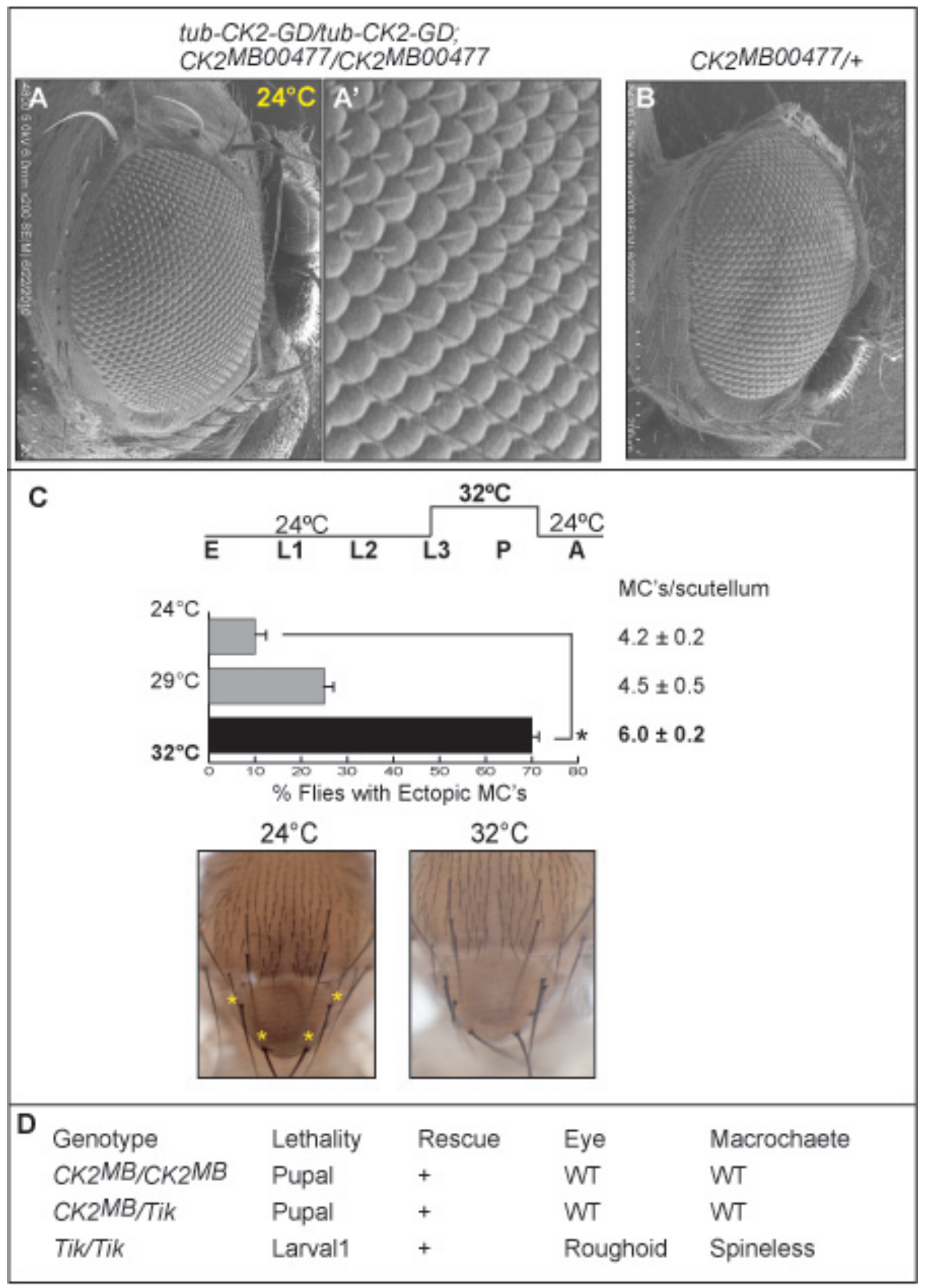


Fig. 7. Phenotypic analysis of rescue flies at restrictive temperature. (A-B) Adult eye phenotypes. The genotypes are (A, A') tub-CK2-G ${ }^{89} D / t u b-C K 2-G^{89} D ; C K 2^{M B 00477} / C K 2^{M B 00477}$, (B) $C K 2^{M B 00477} /+$ magnifications are (A) 200X and (B) 1000X. (C) Quantitative analysis of ectopic MC defects at specific temperatures. Average scutellar $\mathrm{MC}$ count is indicated on the right. Asterisk indicates $P$-value $<0.01$ Adult nota of rescue flies at $24^{\circ}$ and $32^{\circ} \mathrm{C}$. Yellow asterisk indicates the invariant positions of the scutellar macrochaetes. (D) Summary of rescue of the CK2 $\alpha$ mutants by CK2$G^{89} D$ allele. 


\section{Future Perspectives}

The studies indicate that protein kinase CK2 and the phosphatase PP2A play opposing roles in Notch signaling. These roles manifest during the process of lateral inhibition, whereby single R8's or SOP's are selected from proneural clusters during eye and bristle development, respectively. In addition, the data indicates that the opposing roles of CK2 and PP2A might impinge on the $\mathrm{E}(\mathrm{spl})$ members, such as M8, M5, M7 and M $\gamma$. It, therefore, appears that the activities of these repressors is a controlled process, wherein phosphorylation favors a conformational change into an active state, whereas dephosphorylation favors the maintenance of, or the conversion into, the 'autoinhibited' state. Given that the phosphorylation sites in $\mathrm{E}(\mathrm{spl})$ proteins have been conserved through $\sim 50 \mathrm{myr}$ of Drosophila evolution, and the close correspondence of the phosphorylation sites in human/murine Hes6, regulated conformational switching may be applicable to mammalian neurogenesis, as well.

A number of questions arise from the work described in this dissertation. Of immediate relevance is whether PP2A serves to prevent premature activation of M8 during R8 patterning, or if it acts after R8 specification to rapidly deactivate phosphorylated M8. Alternatively, PP2A may act at both stages, thereby exerting control over the timing of the onset of repression, as well as its duration. A second question that remains to be addressed is the role of CK2 and PP2A in post-R8 and -SOP steps. In the eye, Notch signaling is required for specification of the binary R2/R5, R3/R4, R1/R6 cell fates, and for the refinement of five cells of the R7 equivalence group into a single R7 photoreceptor and four cone cells. All of these cell fate specifications require the $\mathrm{E}(\mathrm{spl})$ repressors. It should thus be of interest to determine which of these steps is regulated by CK2 and PP2A, and the E(spl) member that mediates these effects. A similar analysis could be conducted to assess if CK2 and PP2A play reiterated roles in post-SOP steps, specifically the two asymmetric divisions that give rise 
to the four sister cell fates that characterize the bristle sensory organ. Another question that arises is that if $M 8, M 7, M 5$ and $M \gamma$ are similarly regulated, why are $M 3, M \delta$ and $M \beta$ independent of phosphorylation. Studies to address this question will have to await precise lesions in these transcription units and information on their developmental defects.

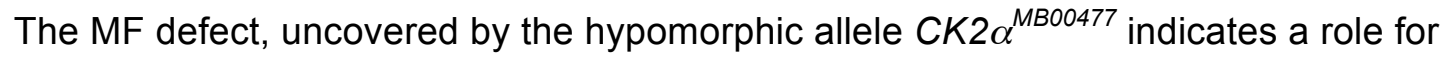
this kinase in either the initiation or the reincarnation of this wave of cell specification. It is noteworthy that the MF defect of $C K 2 \alpha^{M B 00477}$ has been recapitulated by expression of CK2 $\alpha$ RNAi constructs, indicating that this defect is linked to reduced dosage or activity of CK2. Notch and Wingless/Wnt signaling are crucial regulators of the MF, and studies in Drosophila and Xenopus indicate a role for CK2 in both of these signaling pathways. It will be of interest to define the mechanism by which $C K 2 \alpha^{M B 00477}$ elicits MF defects. One possible route is to conduct screens for enhancers or suppressors of the MF defects of CK2 $\alpha^{\text {MB00477 }}$ homozygotes, and then exploit the wealth of resources (mutants, enhancer traps, antibodies, etc.) to better define the underlying mechanism.

A long term, and perhaps a more difficult question that remains to be addressed is control over the phosphorylation cascade. Studies in Drosophila and mammals indicate that PP2A activity is, indeed, modulated by the stage/tissue specific expression of regulatory subunits, such as $\mathrm{Wdb}$. In contrast, the regulation of CK2 still remains obscure. This enzyme is ubiquitously expressed and does not appear to be regulated by second messengers. This, by no means, should diminish its importance, because the activity of this kinase is vital for a number of temporal events. The best example is, perhaps, the circadian clock. In addition, CK2 is essential for cell cycle progression and checkpoint control, and during development, such as embryogenesis and retinal histogenesis. It could be argued that CK2 functions in these processes are essentially limited by the availability of its substrates, but all evidence indicates this to not be the case. For example, co-expression of M8 and CK2 in the MF does 
not elicit dominant loss of R8's, and overexpression in lateral neurons does not accelerate the circadian clock even in the presence of pre-formed Period. One possibility is that CK2 exists in multi-protein complexes. For example, CK2 $\alpha$ directly interacts with the PP2A core dimer, whereas CK2 $\beta$ interacts with the scaffolding subunit. The possibility thus arises that controlled disassembly of CK2 subunits from components of PP2A may permit the formation of the CK2 holoenzyme, thereby leading to phosphorylation of target proteins. While such studies could be conducted in cells in tissue culture, the possibility remains that analysis of these dynamic shifts in enzyme populations may require an in vivo context, where spatial signals such as Notch, EGFR, Wg/Wnt are required.

It is anticipated that studies along these lines will identify additional components and detail the mechanism by which phosphorylation regulates repression by $E(s p l)$ proteins. Studies in Drosophila, a simpler model organism, may serve as a paradigm for regulated repression in development, extending beyond the eye, and provide new insights into aberrant Notch signaling and human disease states. 


\section{Bibliography}

Abdelilah-Seyfried, S., Chan, Y. M., Zeng, C., Justice, N. J., Younger-Shepherd, S., Sharp, L. E., Barbel, S., Meadows, S. A., Jan, L. Y. and Jan, Y. N. (2000). A gain-offunction screen for genes that affect the development of the Drosophila adult external sensory organ. Genetics 155, 733-752.

Akten, B., Jauch, E., Genova, G. K., Kim, E. Y., Edery, I., Raabe, T. and Jackson, F. R. (2003). A role for CK2 in the Drosophila circadian oscillator. Nat Neurosc 6, 251-257.

Alifragis, P., Poortinga, G., Parkhurst, S. M. and Delidakis, C. (1997). A network of interacting transcriptional regulators involved in Drosophila neural fate specification revealed by the yeast two-hybrid system. Proc Natl Acad Sci U S A 94, 13099-13104.

Artavanis-Tsakonas, S., Rand, M. D. and Lake, R. J. (1999). Notch signaling: cell fate control and signal integration in development. Science 284, 770-776.

Bailey, A. M. and Posakony, J. W. (1995). Suppressor of hairless directly activates transcription of enhancer of split complex genes in response to Notch receptor activity. Genes \& Dev 9, 2609-2622.

Bajpai, R., Makhijani, K., Rao, P. R. and Shashidhara, L. S. (2004). Drosophila Twins regulates Armadillo levels in response to Wg/Wnt signal. Development 131, 1007-1016.

Baker, N. E., Yu, S. and Han, D. (1996). Evolution of proneural atonal expression during distinct regulatory phases in the developing Drosophila eye. Curr Biol 6, 1290-1301.

Bandhakavi, S., McCann, R. O., Hanna, D. E. and Glover, C. V. (2002). A positive feedback loop between protein kinase CKII and Cdc37 promotes the activity of multiple protein kinases. J Biol Chem 278, 2829-2836.

Baonza, A. and Freeman, M. (2001). Notch signalling and the initiation of neural development in the Drosophila eye. Develop 128, 3889-3898.

Barford, D., Das, A. K. and Egloff, M. P. (1998). The structure and mechanism of protein phosphatases: insights into catalysis and regulation. Annu Rev Biophys Biomol Struct 27, 133-64.

Belanger-Jasmin, S., Llamosas, E., Tang, Y., Joachim, K., Osiceanu, A. M., Jhas, S. and Stifani, S. (2007). Inhibition of cortical astrocyte differentiation by Hes6 requires amino- and carboxy-terminal motifs important for dimerization and phosphorylation. J Neurochem 103, 2022-34.

Bellen, H. J., Levis, R. W., Liao, G., He, Y., Carlson, J. W., Tsang, G., Evans-Holm, M., Hiesinger, P. R., Schulze, K. L., Rubin, G. M. et al. (2004). The BDGP gene disruption project: single transposon insertions associated with $40 \%$ of Drosophila genes. Genetics 167 , 761-781.

Berdnik, D., Torok, T., Gonzalez-Gaitan, M. and Knoblich, J. A. (2002). The endocytic protein alpha-Adaptin is required for numb-mediated asymmetric cell division in Drosophila. Dev Cell 3, 221-31.

Bessa, J., Gebelein, B., Pichaud, F., Casares, F. and Mann, R. S. (2002). Combinatorial control of Drosophila eye development by eyeless, homothorax, and teashirt. Genes \& Dev 16, 2415-2427. 
Beverly, S. M. and Wilson, A. C. (1984). Molecular evolution in Drosophila and the higher Diptera II. A time scale for fly evolution. J Mol Evol 21, 1-13.

Bidwai, A. P., Hanna, D. E. and Glover, C. V. C. (1992). The free catalytic subunit of casein kinase II is not toxic in vivo. J Biol Chem 267, 18790-18796.

Bidwai, A. P., Reed, J. C. and Glover, C. V. C. (1993). The phosphorylation of Calmodulin by the catalytic subunit of casein kinase II is inhibited by the regulatory subunit. Arch Biochem Biophys 300, 265-270.

Bidwai, A. P., Reed, J. C. and Glover, C. V. C. (1994). Casein kinase II of Saccharomyces cerevisiae contains two distinct regulatory subunits, beta and beta'. Arch Biochem Biophys 309, 348-355.

Bidwai, A. P., Reed, J. C. and Glover, C. V. C. (1995). Cloning and disruption of CKB1, the gene encoding the 38-kDa beta subunit of Saccharomyces cerevisiae casein kinase II (CKII). Deletion of CKII regulatory subunits elicits a salt-sensitive phenotype. J Biol Chem 270, 10395-10404.

Bidwai, A. P., Saxena, A., Zhao, W., McCann, R. O. and Glover, C. V. C. (2000). Multiple, closely spaced alternative $5^{\prime}$ exons in the DmCKIlbeta gene of Drosophila melanogaster. Mol Cell Biol Res Comm 3, 283-291.

Bier, E. (2005). Drosophila, the golden bug, emerges as a tool for human genetics. Nat Rev Genet 6, 9-23.

Birnbaum, M. J., Wu, J., O'Reilley, D. R., Rivera-Marrero, C. A., Hanna, D. E., Miller, L. K. and Glover, C. V. C. (1992). Expression, purification and characterization of Drosophila casein kinase II using the baculovirus system. Protein Expr Purific 3, 142-150.

Boldyreff, B., Meggio, F., Pinna, L. A. and Issinger, O. G. (1993). Reconstitution of normal and hyperactivated forms of casein kinase-2 by variably mutated beta-subunits. Biochem $\mathbf{3 2}$, 12672-7.

Bose, A., Kahali, B., Zhang, S., Lin, J.-M., Allada, R., Karandikar, U. and Bidwai, A. (2006). Drosophila CK2 regulates lateral-inhibition during eye and bristle development. Mech Dev 123, 649-664.

Brand, A. H. and Perrimon, N. (1993). Targeted gene expression as a means of altering cell fates and generating dominant phenotypes. Develop 118, 401-415.

Brand, M. and Campos-Ortega, J. A. (1990). Second-site modifiers of the split mutation of Notch defines genes involved in neurogenesis in Drosophila melanogaster. Dev Genes Evol $198,275-285$.

Bray, S. J. (1997). Expression and function of Enhancer of split bHLH proteins during Drosophila neurogenesis. Perspect Dev Neurobiol 4, 313-323.

Bray, S. J. (2006). Notch signalling: a simple pathway becomes complex. Nat Rev Mol Cell Biol 7, 678-89.

Brou, C., Logeat, F., Gupta, N., Bessia, C., LeBail, O., Doedens, J. R., Cumano, A., Roux, P., Black, R. A. and Israel, A. (2000). A novel proteolytic cleavage involved in Notch signaling: the role of the disintegrin-metalloprotease TACE. Mol Cell 5, 207-16.

Bruckner, K., Perez, L., Clausen, H. and Cohen, S. (2000). Glycosyltransferase activity of Fringe modulates Notch-Delta interactions. Nature 406, 411-5. 
Buchou, T., Vernet, M., Blond, O., Jensen, H., Pointu, H., Olsen, B., Cochet, C., Issinger, O. and Boldyreff, B. (2003). Disruption of the regulatory beta subunit of protein kinase CK2 in mice leads to a cell-autonomous defect and early embryonic lethality. Mol Cell Biol 23, 908915.

Burnett, G. and Kennedy, E. P. (1954). The enzymatic phosphorylation of proteins. J Biol Chem 211, 969-980.

Cagan, R. L. and Ready, D. F. (1989). Notch is required for successive cell decisions in the developing Drosophila retina. Genes \& Dev 3, 1099-1112.

Calleja, M., Renaud, O., Usui, K., Pistillo, D., Morata, G. and Simpson, P. (2002). How to pattern an epithelium: lessons from achaete-scute regulation on the notum of Drosophila. Gene 292, 1-12.

Campos-Ortega, J. A. (1997). Neurogenesis in Drosophila: an historical perspective and some prospects. Perspect Dev Neurobiol 4, 267-271.

Campos-Ortega, J. A. (1998). The genetics of the Drosophila achaete-scute gene complex: a historical perspective. Int J Dev Biol 42, 291-297.

Cauchi, R. J. and van den Heuvel, M. (2006). The fly as a model for neurodegenerative diseases: is it worth the jump? Neurodegener Dis 3, 338-56.

Chantalat, L., Leroy, D., Filhol, O., Nueda, A., Benitez, M. J., Chambaz, E. M., Cochet, C. and Dideberg, O. (1999). Crystal structure of the human protein kinase CK2 regulatory subunit reveals its zinc finger-mediated dimerization. EMBO J 18, 2930-2940.

Chanut, F. and Heberlein, U. (1995). Role of the morphogenetic furrow in establishing polarity in the Drosophila eye. Development 121, 4085-94.

Chen, G. and Courey, A. J. (2000). Groucho/TLE family proteins and transcriptional repression. Gene 249, 1-16.

Chen-Wu, L. P. J., Padmanabha, R. and Glover, C. V. C. (1988). Isolation, sequencing, and disruption of the CKA1 gene encoding the alpha subunit of yeast casein kinase II. Mol Cell Biol 8, 4981-4990.

Chitnis, A. (2006). Why is delta endocytosis required for effective activation of notch? Dev Dyn 235, 886-94.

Cho, U. S. and Xu, W. (2007). Crystal structure of a protein phosphatase 2A heterotrimeric holoenzyme. Nature 445, 53-7.

Cochet, C. and Chambaz, E. M. (1983). Oligomeric structure and catalytic activity of G type casein kinase. Isolation of the two subunits and renaturation experiments. J Biol Chem 258, 1403-6.

Cooper, M. T. D. and Bray, S. J. (1999). Frizzled regulation of Notch signalling polarizes cell fate in the Drosophila eye. Nature, 526-530.

Culi, J. and Modolell, J. (1998). Proneural gene self-stimulation in neural precursors: an essential mechanism for sense organ development that is regulated by Notch signaling. Genes \& Dev 12, 2036-2047.

Dahmus, G. K., Glover, C. V. C., Brutlag, D. and Dahmus, M. E. (1984). Similarities in structure and function of calf thymus and Drosophila casein kinase II. J Biol Chem 259, 90019006. 
Dambly-Chaudiere, C. and Vervoort, M. (1998). The bHLH genes in neural development. Int J Dev Biol 42, 269-273.

de Celis, J. F., Barrio, R., del Arco, A. and Garcia-Bellido, A. (1993). Genetic and molecular characterization of a Notch mutation in its Delta- and Serrate-binding domain in Drosophila. Proc Natl Acad Sci U S A 90, 4037-41.

de Celis, J. F. and Bray, S. J. (2000). The Abruptex domain of Notch regulates negative interactions between Notch, its ligands and Fringe. Development 127, 1291-302.

de Celis, J. F. and Garcia-Bellido, A. (1994). Modifications of the notch function by Abruptex mutations in Drosophila melanogaster. Genetics 136, 183-94.

Delidakis, C. and Artavanis-Tsakonas, S. (1991). The enhancer of split [E(spl)] locus of Drosophila encodes seven independant helix-loop-helix proteins. Proc Natl Acad Sci U S A 89, 8731-8735.

Denu, J. M., Stuckey, J. A., Saper, M. A. and Dixon, J. E. (1996). Form and function in protein dephosphorylation. Cell 87, 361-4.

Eichhorn, P. J., Creyghton, M. P. and Bernards, R. (2009). Protein phosphatase 2A regulatory subunits and cancer. Biochim Biophys Acta 1795, 1-15.

Emery, G., Hutterer, A., Berdnik, D., Mayer, B., Wirtz-Peitz, F., Gaitan, M. G. and Knoblich, J. A. (2005). Asymmetric Rab 11 endosomes regulate delta recycling and specify cell fate in the Drosophila nervous system. Cell 122, 763-73.

Fanto, M. and Mlodzik, M. (1999). Asymmetric Notch activation specifies photoreceptors R3 and R4 and planar polarity in the drosophila eye. Nature 397, 523-526.

Fischer, E. H. and Krebs, E. G. (1955). Conversion of phosphorylase b to phosphorylase a in muscle extracts. J Biol Chem 216, 121-32.

Fleming, R. J. (1998). Structural conservation of Notch receptors and ligands. Semin Cell Dev Biol 9, 599-607.

Fleming, R. J., Gu, Y. and Hukriede, N. A. (1997). Serrate-mediated activation of Notch is specifically blocked by the product of the gene fringe in the dorsal compartment of the Drosophila wing imaginal disc. Development 124, 2973-81.

Frankfort, B. J. and Mardon, G. (2002). R8 development in the Drosophila eye: a paradigm for neural selection and differentiation. Develop 129, 1295-1306.

Frankfort, B. J., Nolo, R., Zhang, Z., Bellen, H. and Mardon, G. (2001). senseless repression of rough is required for $\mathrm{R} 8$ photoreceptor differentiation in the developing Drosophila eye. Neuron 32, 403-414.

Frise, E., Knoblich, J. A., Younger-Shepherd, S., Jan, L. Y. and Jan, Y. N. (1996). The Drosophila Numb protein inhibits signaling of the Notch receptor during cell-cell interaction in sensory organ lineage. Proc Natl Acad Sci U S A 93, 11925-11932.

Fryer, C. J., Lamar, E., Turbachova, I., Kintner, C. and Jones, K. A. (2002). Mastermind mediates chromatin-specific transcription and turnover of the Notch enhancer complex. Genes Dev 16, 1397-411.

Fryxell, K. J., Soderlund, M. and Jordan, T. V. (2001). An animal model for the molecular genetics of CADASIL. (Cerebral autosomal dominant arteriopathy with subcortical infarcts and leukoencephalopathy). Stroke 32, 6-11. 
Giagtzoglou, N., Alifragis, P., Koumbanakis, K. A. and Delidakis, C. (2003). Two modes of recruitment of $\mathrm{E}(\mathrm{spl})$ repressors onto target genes. Develop 130, 259-270.

Gibert, J. M. and Simpson, P. (2003). Evolution of cis-regulation of the proneural genes. Int J Dev Biol 47, 643-651.

Giebel, B. and Campos-Ortega, J. A. (1997). Functional dissection of the Drosophila enhancer of split protein, a suppressor of neurogenesis. Proc Natl Acad Sci U S A 94, 62506254.

Giot, L., Bader, J. S., Brouwer, C., Chaudhuri, A., Kuang, B., Li, Y., Hao, Y. L., Ooi, C. E., Godwin, B., Vitols, E. et al. (2003). A protein interaction map of Drosophila melanogaster. Science 302, 1727-1736.

Glover, C. V. C., Shelton, E. R. and Brutlag, D. L. (1983). Purification and characterization of a type II casein kinase from Drosophila melanogaster. J Biol Chem 258, 3258-3265.

Goldberg, Y. (1999). Protein phosphatase 2A: who shall regulate the regulator? Biochem Pharmacol 57, 321-8.

Goldstein, A. and Lampen, J. O. (1976). b-D-fructofuranoside fructohydrolase from yeast. Meth Enzymol 76, 504-511.

Gordon, W. R., Arnett, K. L. and Blacklow, S. C. (2008). The molecular logic of Notch signaling--a structural and biochemical perspective. J Cell Sci 121, 3109-19.

Gordon, W. R., Vardar-Ulu, D., Histen, G., Sanchez-Irizarry, C., Aster, J. C. and Blacklow, S. C. (2007). Structural basis for autoinhibition of Notch. Nat Struct Mol Biol 14, 295-300.

Gratton, M.-O., Torban, E., Jasmin, S. B., Theriault, F. M., German, M. S. and Stifani, S. (2003). Hes6 Promotes Cortical Neurogenesis and Inhibits Hes1 Transcription Repression Activity by Multiple Mechanisms. Mol Cell Biol 23, 6922-6935.

Gridley, T. (1997). Notch signaling in vertebrate development and disease. Mol Cell Neurosci 9, 103-8.

Guo, M., Jan, L. Y. and Jan, Y. N. (1996). Control of daughter cell fates during asymmetric division: interaction of Numb and Notch. Neuron 17, 27-41.

Gupta-Rossi, N., Six, E., LeBail, O., Logeat, F., Chastagner, P., Olry, A., Israel, A. and Brou, C. (2004). Monoubiquitination and endocytosis direct gamma-secretase cleavage of activated Notch receptor. J Cell Biol 166, 73-83.

Haines, N. and Irvine, K. D. (2003). Glycosylation regulates Notch signalling. Nat Rev Mol Cell Biol 4, 786-97.

Hanks, S. K. and Quinn, A. M. (1991). Protein kinase catalytic domain sequence database: identification of conserved features of primary structure and classification of family members. Meth Enzymol 200, 38-61.

Hanna, D. E., Rethinaswamy, A. and Glover, C. V. C. (1995). Casein kinase II is required for cell cycle progression during G1 and G2/M in Saccharomyces cerevisiae. J Biol Chem 270, 25905-25914.

Hannus, M., Feiguin, F., Heisenberg, C. P. and Eaton, S. (2002). Planar cell polarization requires Widerborst, a B' regulatory subunit of protein phosphatase 2A. Develop 129, 34933503.

Hartenstein, V. and Posakony, J. W. (1990). A dual function of the Notch gene in Drosophila sensillum development. Dev Biol 142, 13-30. 
Hartley, D. A., Preiss, A. and Artavanis-Tsakonas, S. (1988). A deduced gene product from the Drosophila neurogenic locus, Enhancer of split, shows homology to mammalian G-protein beta subunit. Cell 55, 785-795.

Hartley, D. A., Xu, T. A. and Artavanis-Tsakonas, S. (1987). The embryonic expression of the Notch locus of Drosophila melanogaster and the implications of point mutations in the extracellular EGF-like domain of the predicted protein. EMBO J 6, 3407-17.

Hathaway, G. M., Lubben, T. H. and Traugh, J. A. (1980). Inhibition of casein kinase II by heparin. J Biol Chem 255, 8038-8041.

Hathaway, G. M. and Traugh, J. A. (1979). Cyclic Nucleotide-independant Protein Kinase from Rabbit Reticulocytes. Purification of Casein Kinases. J Biol Chem 254, 762-768.

Heitzler, P., Bourouis, M., Ruel, L., Carteret, C. and Simpson, P. (1996). Genes of the Enhancer of split and achaete-scute complexes are required for a regulatory loop between Notch and Delta during lateral signalling in Drosophila. Develop 122, 161-171.

Heriche, J. K., Lebrin, F., Rabilloud, T., Leroy, D., Chambaz, E. M. and Goldberg, Y. (1997). Regulation of protein phosphatase 2A by direct interaction with casein kinase 2alpha. Science 276, 952-955.

Hicks, C., Johnston, S. H., diSibio, G., Collazo, A., Vogt, T. F. and Weinmaster, G. (2000). Fringe differentially modulates Jagged 1 and Delta1 signalling through Notch1 and Notch2. Nat Cell Biol 2, 515-20.

Hicks, C., Ladi, E., Lindsell, C., Hsieh, J. J., Hayward, S. D., Collazo, A. and Weinmaster, G. (2002). A secreted Delta1-Fc fusion protein functions both as an activator and inhibitor of Notch1 signaling. J Neurosci Res 68, 655-67.

Hori, K., Fostier, M., Ito, M., Fuwa, T. J., Go, M. J., Okano, H., Baron, M. and Matsuno, K. (2004). Drosophila deltex mediates suppressor of Hairless-independent and late-endosomal activation of Notch signaling. Development 131, 5527-37.

Hsiung, F. and Moses, K. (2002). Retinal development in Drosophila: specifying the first neuron. Hum Mol Genet 11, 1207-1214.

Huang, Y. and Fischer-Vize, J. (1996). Undifferentiated cells in the developing Drosophila eye influence facet assembly and require the Fat facets ubiquitin-specific protease. Develop 122, 3207-3216.

Ilagan, M. X. and Kopan, R. (2007). Notch signaling pathway. Cell 128, 1246.

Itoh, M., Kim, C. H., Palardy, G., Oda, T., Jiang, Y. J., Maust, D., Yeo, S. Y., Lorick, K., Wright, G. J., Ariza-McNaughton, L. et al. (2003). Mind bomb is a ubiquitin ligase that is essential for efficient activation of Notch signaling by Delta. Dev Cell 4, 67-82.

Jafar-Nejad, H., Acar, M., Nolo, R., Lacin, H., Pan, H., Parkhurst, S. M. and Bellen, H. J. (2003). Senseless acts as a binary switch during sensory organ precursor selection. Genes \& Dev 17, 2966-2678.

Jafar-Nejad, H., Andrews, H. K., Acar, M., Bayat, V., Wirtz-Peitz, F., Mehta, S. Q., Knoblich, J. A. and Bellen, H. J. (2005). Sec15, a component of the exocyst, promotes notch signaling during the asymmetric division of Drosophila sensory organ precursors. Dev Cell 9, 351-63.

Jafar-Nejad, H. and Bellen, H. J. (2004). Gfi/Pag-3/senseless zinc finger proteins: a unifying theme? Mol Cell Biol 24, 8803-8812. 
Jaffe, L., Ryoo, H.-D. and Mann, R. S. (1997). A role for phosphorylation by casein kinase II in modulating Antennapedia activity in Drosophila. Genes \& Dev 11, 1327-1340.

Janssens, V. and Goris, J. (2001). Protein phosphatase 2A: a highly regulated family of serine/threonine phosphatases implicated in cell growth and signalling. Biochem J 353, 41739.

Jarman, A., Sun, Y., Jan, L. and Jan, Y. (1995). Role of the proneural gene, atonal, in formation of Drosophila chordotonal organs and photoreceptors. Develop 121, 2019-2030.

Jarman, A. P. and Ahmed, I. (1998). The specificity of proneural genes in determining Drosophila sense organ identity. Mech Dev 76, 117-25.

Jarman, A. P., Grell, E. H., Ackerman, L., Jan, L. Y. and Jan, Y. N. (1994). atonal is the proneural gene for Drosophila photoreceptors. Nature 369, 398-400.

Jauch, E., Melzig, J., Brkulj, M. and Raabe, T. (2002). In vivo functional analysis of Drosophila protein kinase casein kinase 2 (CK2) beta-subunit. Gene 298, 29-39.

Jauch, E., Wecklein, H., Stark, F., Jauch, M. and Raabe, T. (2006). The Drosophila melanogaster DmCK2beta transcription unit encodes for functionally non-redundant protein isoforms. Gene 374, 142-152.

Jennings, B. H., Tyler, D. M. and Bray, S. J. (1999). Target Specificities of Drosophila Enhancer of split Basic Helix-Loop-Helix Proteins. Mol Cell Biol 19, 4600-4610.

Jimenez, G. and Ish-Horowicz, D. (1997). A chimeric Enhancer of split transcriptional activator drives neural development and achaete-scute expression. Mol Cell Biol 17, 43554362.

Joutel, A., Corpechot, C., Ducros, A., Vahedi, K., Chabriat, H., Mouton, P., Alamowitch, S., Domenga, V., Cecillion, M., Marechal, E. et al. (1996). Notch3 mutations in CADASIL, a hereditary adult-onset condition causing stroke and dementia. Nature 383, 707-10.

Junttila, M. R., Li, S. P. and Westermarck, J. (2008). Phosphatase-mediated crosstalk between MAPK signaling pathways in the regulation of cell survival. FASEB J 22, 954-65.

Kahali, B., Bose, A., Karandikar, U., Bishop, C. P. and Bidwai, A. (2009). On the mechanism underlying the divergent retinal and bristle defects of $\mathrm{M}^{*}(E(s p l) D)$ in Drosophila. Genesis 47, 456-468.

Kahali, B., Kim, J., Karandikar, U., Bishop, C. P. and Bidwai, A. P. (2010). Evidence that the C-terminal domain (CtD) autoinhibits neural repression by Drosophila E(spl)M8. Genesis 48, 44-55.

Karandikar, U., Trott, R. L., Yin, J., Bishop, C. P. and Bidwai, A. P. (2004). Drosophila CK2 regulates eye morphogenesis via phosphorylation of E(spl)M8. Mech Dev 121, 273-286.

Kelley, M. R., Kidd, S., Deutsch, W. A. and Young, M. W. (1987). Mutations altering the structure of epidermal growth factor-like coding sequences at the Drosophila Notch locus. Cell 51, 539-48.

Klambt, C., Knust, E., Tietze, K. and Campos-Ortega, J. A. (1989). Closely related transcripts encoded by the neurogenic gene complex Enhancer of split of Drosophila melanogaster. EMBO J 8, 203-210.

Klein, T. and Arias, A. M. (1998). Interactions among Delta, Serrate and Fringe modulate Notch activity during Drosophila wing development. Development 125, 2951-62. 
Knust, E., Bremer, K. A., Vassin, H., Ziemer, A., Tepass, U. and Campos-Ortega, J. A. $(1987)$. The enhancer of split locus and neurogenesis in Drosophila melanogaster. Dev Biol 122, 262-73.

Knust, E., Schrons, H., Grawe, F. and Campos-Ortega, J. A. (1992). Seven genes of the enhancer of split complex of Drosophila melanogaster encode helix-loop-helix proteins. Genetics 132, 505-518.

Kovall, R. A. and Hendrickson, W. A. (2004). Crystal structure of the nuclear effector of Notch signaling, CSL, bound to DNA. EMBO J 23, 3441-51.

Kuenzel, E. A. and Krebs, E. G. (1985). A synthetic substrate specific for casein kinase II. Proc Natl Acad Sci U S A 82, 737-741.

Kuenzel, E. A., Mulligan, J. A., Sommercorn, J. and Krebs, E. G. (1987). Substrate specificity determinants for casein kinase II as deduced from studies with synthetic peptides. $J$ Biol Chem 262, 9136-9140.

Kumar, J. P. and Moses, K. (2001). The EGF receptor and notch signaling pathways control the initiation of the morphogenetic furrow during Drosophilaeye development. Develop 128, 2689-2697.

Kuntamalla, P., Kunttas, E., Karandikar, U., Bishop, C. and Bidwai, A. (2009). Drosophila protein kinase CK2 is rendered temperature-sensitive by mutations of highly conserved residues flanking the activation segment. Mol Cell Biochem 323, 49-60.

Kunttas-Tatli, E., Bose, A., Kahali, B., Bishop, C. P. and Bidwai, A. P. (2009). Functional dissection of Timekeeper (Tik) implicates opposite roles for CK2 and PP2A during Drosophila neurogenesis. Genesis 47, 647-658.

Kurooka, H., Kuroda, K. and Honjo, T. (1998). Roles of the ankyrin repeats and C-terminal region of the mouse notch1 intracellular region. Nucleic Acids Res 26, 5448-55.

Kusk, M., Bendixen, C., Duno, M., Westergaard, O. and Thomsen, B. (1995). Genetic dissection of intersubunit contacts within human protein kinase CK2. J Mol Biol 253, 703-711.

Lai, E. C. (2004). Notch signaling: control of cell communication and cell fate. Develop 131, 965-973.

Lai, E. C., Roegiers, F., Qin, X., Jan, Y. N. and Rubin, G. M. (2005a). The ubiquitin ligase Drosophila Mind bomb promotes Notch signaling by regulating the localization and activity of Serrate and Delta. Development 132, 2319-32.

Lai, E. C., Tam, B. and Rubin, G. M. (2005b). Pervasive regulation of Drosophila Notch target genes by GY-box-, Brd-box-, and K-box-class microRNAs. Genes Dev 19, 1067-80.

Le Borgne, R. (2006). Regulation of Notch signalling by endocytosis and endosomal sorting. Curr Opin Cell Biol 18, 213-22.

Le Borgne, R., Bardin, A. and Schweisguth, F. (2005). The roles of receptor and ligand endocytosis in regulating Notch signaling. Development 132, 1751-62.

Le Borgne, R. and Schweisguth, F. (2003). Unequal segregation of Neuralized biases Notch activation during asymmetric cell division. Dev Cell 5, 139-48.

Lecourtois, M. and Schweisguth, F. (1995). The neurogenic suppressor of hairless DNAbinding protein mediates the transcriptional activation of the enhancer of split complex genes triggered by Notch signaling. Genes \& Dev 9, 2598-2608. 
Ledent, V. and Vervoort, M. (2001). The basic helix-loop-helix protein family: Comparative genomics and phylogenetic analysis. Genome Res 11, 754-770.

Lee, Y. S. and Carthew, R. W. (2003). Making a better RNAi vector for Drosophila: use of intron spacers. Methods 30, 322-329.

Lehmann, R., Jimenez, F., Dietrich, U. and Campos-Ortega, J. A. (1983). On the phenotype and development of mutants of early development in Drosophila melanogaster. Roux's Arch Dev Biol 192, 62-74.

Li, X., Scuderi, A., Letsou, A. and Virshup, D. M. (2002). B56-associated protein phosphatase 2A is required for survival and protects from apoptosis in Drosophila melanogaster. Mol Cell Biol 22, 3674-84.

Li, Y., Lei, L., Irvine, K. D. and Baker, N. E. (2003). Notch activity in neural cells triggered by a mutant allele with altered glycosylation. Develop 130, 2829-2840.

Ligoxygakis, P., Yu, S. Y., Delidakis, C. and Baker, N. E. (1998). A subset of Notch functions during Drosophila eye development require $\mathrm{Su}(\mathrm{H})$ and $\mathrm{E}(\mathrm{spl})$ gene complex. Develop 125, 2893-2900.

Lin, J. M., Kilman, V. L., Keegan, K., Paddock, B., Emery-Le, M., Rosbash, M. and Allada, R. (2002). A role for casein kinase 2alpha in the Drosophila circadian clock. Nature 420, 816820.

Lin, W. J., Tuazon, P. T. and Traugh, J. A. (1991). Characterization of the catalytic subunit of casein kinase II expressed in Escherichia coli and regulation of activity. J Biol Chem 266, 5664-5669.

Litchfield, D. W. (2003). Protein kinase CK2: structure, regulation and role in cellular decisions of life and death. Biochem J 369, 1-15.

Logeat, F., Bessia, C., Brou, C., LeBail, O., Jarriault, S., Seidah, N. G. and Israel, A. (1998). The Notch1 receptor is cleaved constitutively by a furin-like convertase. Proc Natl Acad Sci U S A 95, 8108-12.

Lou, D. Y., Dominguez, I., Toselli, P., Landesman-Bollag, E., O'Brien, C. and Seldin, D. C. (2008). The alpha catalytic subunit of protein kinase CK2 is required for mouse embryonic development. Mol Cell Biol 28, 131-9.

Luscher, B., Kuenzel, E. A., Krebs, E. G. and Eisenman, R. N. (1989). Myc oncoproteins are phosphorylated by casein kinase II. EMBO J 8, 1111-9.

Marcus, E. A., Kintner, C. and Harris, W. (1998). The role of GSK3beta in regulating neuronal differentiation in Xenopus laevis. Mol Cell Neurosci 12, 269-80.

Massari, M. E. and Murre, C. (2000). Helix-loop-helix proteins: Regulators of transcription in eukaryotic organisms. Mol Cell Biol 20, 429-440.

McCright, B., Rivers, A.M., Audlin, S., and Virshup, D.M. (1996). The B56 family of protein phosphatase 2A (PP2A) regulatory subunits encodes differentiationinduced phosphoproteins that target PP2A to both nucleus and cytoplasm. J Biol Chem 271, 22081-22089.

McGill, M. A. and McGlade, C. J. (2003). Mammalian numb proteins promote Notch1 receptor ubiquitination and degradation of the Notch1 intracellular domain. J Biol Chem 278, 23196-203.

Meggio, F., Brunati, A. M. and Pinna, L. A. (1983). Autophosphorylation of type 2 casein kinase TS at both its alpha and beta subunits. FEBS Lett 160, 203-208. 
Meggio, F., Donella-Deana, A., Brunati, A. M. and Pinna, L. A. (1982). Inhibition of rat liver cytosol casein kinases by heparin. FEBS Lett 141, 257-262.

Meggio, F. and Pinna, L. A. (1984). Subunit structure and autophosphorylation mechanism of casein kinase-TS (type-2) from rat liver cytosol. Eur J Biochem 145, 593-599.

Meggio, F. and Pinna, L. A. (2003). One-thousand-and-one substrates of protein kinase CK2? FASEB J 17, 349-68.

Metaxakis, A., Oehler, S., Klinakis, A. and Savakis, C. (2005). Minos as a genetic and genomic tool in Drosophila melanogaster. Genetics 171, 571-81.

Miele, L. and Osborne, B. (1999). Arbiter of differentiation and death: Notch signaling meets apoptosis. J Cell Physiol 181, 393-409.

Moloney, D. J., Panin, V. M., Johnston, S. H., Chen, J., Shao, L., Wilson, R., Wang, Y., Stanley, P., Irvine, K. D., Haltiwanger, R. S. et al. (2000). Fringe is a glycosyltransferase that modifies Notch. Nature 406, 369-75.

Moore, K. B., Schneider, M. L. and Vetter, M. L. (2002). Posttranslational mechanisms control the timing of bHLH function and regulate retinal cell fate. Neuron 34, 183-95.

Mumby, M. (2007). The 3D structure of protein phosphatase 2A: new insights into a ubiquitous regulator of cell signaling. ACS Chem Biol 2, 99-103.

Mumm, J. S. and Kopan, R. (2000). Notch signaling: From the outside in. Dev Biol 228, 151165.

Nagel, A., Yu, Y. and Preiss, A. (1999). Enhancer of Split [E(spl)D] is a Gro-independent, hypermorphic mutation in Drosophila. Dev Genet 25, 168-179.

Nagel, A. C. and Preiss, A. (1999). Notch spl is deficient for inductive processes in the eye, and $\mathrm{E}(\mathrm{spl}) \mathrm{D}$ enhances split by interfering with proneural activity. Dev Biol 208, 406-415.

Nakao, K. and Campos-Ortega, J. A. (1996). Persistent expression of genes of the Enhancer of Split Complex suppress neural development in Drosophila. Neuron 16, 275-286.

Nam, Y., Sliz, P., Song, L., Aster, J. C. and Blacklow, S. C. (2006). Structural basis for cooperativity in recruitment of MAML coactivators to Notch transcription complexes. Cell 124, 973-83.

Niefind, K., Guerra, B., Ermakowa, I. and Issinger, O. G. (2001). Crystal structure of human protein kinase CK2: insights into basic properties of the CK2 holoenzyme. EMBO J 20, 53205331.

Niefind, K., Guerra, B., Pinna, L. A., Issinger, O. G. and Schomburg, D. (1998). Crystal structure of the catalytic subunit of protein kinase CK2 from Zea mays at 2.1 A resolution. EMBO J 17, 2451-2462.

Nolo, R., Abbott, L. A. and Bellen, H. J. (2000). Senseless, a Zn finger transcription factor, is necessary and sufficient for sensory organ development in Drosophila. Cell 102, 349-62.

O'Connor-Giles, K. M. and Skeath, J. B. (2003). Numb inhibits membrane localization of Sanpodo, a four-pass transmembrane protein, to promote asymmetric divisions in Drosophila. Dev Cell 5, 231-43.

Okajima, T. and Irvine, K. D. (2002). Regulation of notch signaling by o-linked fucose. Cell 111, 893-904. 
Okajima, T., Xu, A. and Irvine, K. D. (2003). Modulation of notch-ligand binding by protein Ofucosyltransferase 1 and fringe. J Biol Chem 278, 42340-5.

Okochi, M., Steiner, H., Fukumori, A., Tanii, H., Tomita, T., Tanaka, T., Iwatsubo, T., Kudo, T., Takeda, M. and Haass, C. (2002). Presenilins mediate a dual intramembranous gamma-secretase cleavage of Notch-1. EMBO J 21, 5408-16.

ole-MoiYoi, O. K. (1995). Casein kinase II in theileriosis. Science 267, 834-836.

ole-MoiYoi, O. K., Brown, W. C., lams, K. P., Nayar, A., Tsukamoto, T. and Macklin, M. D. (1993). Evidence for the induction of casein kinase II in bovine lymphocytes transformed by the intracellular protozoan parasite Theileria parva. EMBO J 12, 1621-31.

Overstreet, E., Fitch, E. and Fischer, J. A. (2004). Fat facets and Liquid facets promote Delta endocytosis and Delta signaling in the signaling cells. Development 131, 5355-66.

Padmanabha, R., Chen-Wu, J. L. P., Hanna, D. E. and Glover, C. V. C. (1990). Isolation, sequencing, and disruption of the yeast CKA2 gene: casein kinase II is essential for viability in Saccharomyces cerevisiae. Mol Cell Biol 10, 4089-4099.

Panin, V. M., Papayannopoulos, V., Wilson, R. and Irvine, K. D. (1997). Fringe modulates Notch-ligand interactions. Nature 387, 908-12.

Parks, A. L., Turner, R. and Muskavitch, M. A. T. (1995). Relationship between complex Delta expression and the specification of retinal cell fates during Drosophila eye development. Mech Dev 50, 201-216.

Paroush, Z., Finley, R. L., Kidd, T., Wainwright, S. M., Ingham, P. W., Brent, R. and IshHorowcz, D. (1994). Groucho is required for Drosophila neurogenesis, segmentation, and sex determination and interacts directly with hairy related bHLH proteins. Cell 79, 805-815.

Pei, Z. and Baker, N. E. (2008). Competition between Delta and the Abruptex domain of Notch. BMC Dev Biol 8, 4.

Pepperkok, R., Lorenz, P., Ansorge, W. and Pyerin, W. (1994). Casein kinase II is required for transition of G0/G1, early G1, and G1/S phases of the cell cycle. J Biol Chem 269, 69866991.

Pepple, K. L., Atkins, M., Venken, K., Wellnitz, K., Harding, M., Frankfort, B. and Mardon, G. (2008). Two-step selection of a single R8 photoreceptor: a bistable loop between senseless and rough locks in R8 fate. Development 135, 4071-4079.

Petcherski, A. G. and Kimble, J. (2000). Mastermind is a putative activator for Notch. Curr Biol 10, R471-3.

Pinna, L. A. (2002). Protein kinase CK2: a challenge to canons. J Cell Sci 115, 3873-8.

Poodry, C. A. (1990). shibire, a neurogenic mutant of Drosophila. Dev Biol 138, 464-72.

Poulson, D. F. (1937). Chromosomal Deficiencies and the Embryonic Development of Drosophila Melanogaster. Proc Natl Acad Sci U S A 23, 133-7.

Rasmussen, T., Skjøth, I. H. E., Jensen, H. H., Niefind, K., Boldyreff, B. and Issinger, O. G. (2005). Biochemical characterization of the recombinant human Drosophila homologues Timekeeper and Andante involved in the Drosophila circadian oscillator. Mol Cell Biochem 274, 151-161.

Ready, D. F., Hanson, T. E. and Benzer, S. (1976). Development of the Drosophila retina, a neurocrystalline lattice. Dev Biol 53, 217-240. 
Rebay, I., Fleming, R. J., Fehon, R. G., Cherbas, L., Cherbas, P. and ArtavanisTsakonas, S. (1991). Specific EGF repeats of Notch mediate interactions with Delta and Serrate: implications for Notch as a multifunctional receptor. Cell 67, 687-99.

Reed, J. C., Bidwai, A. P. and Glover, C. V. C. (1994). Cloning and disruption of CKB2, the gene encoding the $32 \mathrm{kDa}$ regulatory beta' subunit of Saccharomyces cerevisiae casein kinase II. J Biol Chem 269, 18192-18200.

Rethinaswamy, A., Birnbaum, M. J. and Glover, C. V. C. (1998). Temperature-sensitive mutations of the CKA1 gene reveal a role for casein kinase II in maintenance of cell polarity in Saccharomyces cerevisiae. J Biol Chem 273, 5869-5877.

Santolini, E., Puri, C., Salcini, A. E., Gagliani, M. C., Pelicci, P. G., Tacchetti, C. and Di Fiore, P. P. (2000). Numb is an endocytic protein. J Cell Biol 151, 1345-52.

Sasai, Y., Kageyama, R., Tagawa, Y., Shigemoto, R. and Nakanishi, S. (1992). Two mammalian helix-loop-helix factors structurally related to Drosophila hairy and Enhancer of split. Genes Dev 6, 2620-34.

Sasamura, T., Sasaki, N., Miyashita, F., Nakao, S., Ishikawa, H. O., Ito, M., Kitagawa, M., Harigaya, K., Spana, E., Bilder, D. et al. (2003). neurotic, a novel maternal neurogenic gene, encodes an O-fucosyltransferase that is essential for Notch-Delta interactions. Development $130,4785-95$.

Sathyanarayanan, S., Zheng, X., Xiao, R. and Sehgal, A. (2004). Posttranslational regulation of Drosophila PERIOD protein by protein phosphatase 2A. Cell 116, 603-615.

Saxena, A., Padmanabha, R. and Glover, C. V. C. (1987). Isolation and sequencing of cDNA clones encoding $\mathrm{a}$ and $\mathrm{b}$ subunits of Drosophila melanogaster casein kinase II. Mol Cell Biol 7, 3409-3417.

Schreiber, S. L., Preiss, A., Nagel, A. C., Wech, I. and Maier, D. (2002). Genetic screen for modifiers of the rough eye phenotype resulting from overexpression of the Notch antagonist hairless in Drosophila. Genesis 33, 141-52.

Schrons, H., Knust, E. and Campos-Ortega, J. A. (1992). The enhancer of split complex and adjacent genes in the $96 \mathrm{~F}$ region of Drosophila melanogaster are required for segregation of neural and epidermal cells. Genetics 132, 481-503.

Schweisguth, F. and Posakony, J. W. (1992). Suppressor of hairless, the Drosophila homolog of the mouse recombination signal-binding protein gene, controls sensory organ cell fates. Cell 69, 1199-1212.

Seldin, D. C., Lou, D. Y., Toselli, P., Landesman-Bollag, E. and Dominguez, I. (2008). Gene targeting of CK2 catalytic subunits. Mol Cell Biochem 316, 141-7.

Seugnet, L., Simpson, P. and Haenlin, M. (1997). Requirement for dynamin during Notch signaling in Drosophila neurogenesis. Dev Biol 192, 585-98.

Shao, L., Moloney, D. J. and Haltiwanger, R. (2003). Fringe modifies O-fucose on mouse Notch1 at epidermal growth factor-like repeats within the ligand-binding site and the Abruptex region. J Biol Chem 278, 7775-82.

Shepard, S. B., Broverman, S. A. and Muskavitch, M. A. (1989). A tripartite interaction among alleles of Notch, Delta, and Enhancer of split during imaginal development of Drosophila melanogaster. Genetics 122, 429-38. 
Sherwood, D. R. and McClay, D. R. (1997). Identification and localization of a sea urchin Notch homologue: insights into vegetal plate regionalization and Notch receptor regulation. Development 124, 3363-74.

Shi, Y. (2009). Serine/Threonine Phosphatases: Mechanism through Structure. Cell 139, 468484.

Shiomi, K., Takeichi, M., Nishida, Y., Nishi, Y. and Uemura, T. (1994). Alternative cell fate choice induced by low-level expression of a regulator of protein phosphatase $2 \mathrm{~A}$ in the Drosophila peripheral nervous system. Develop 120, 1591-1599.

Silverstein, A. M., Barrow, C. A., Davis, A. J. and Mumby, M. C. (2002). Actions of PP2A on the MAP kinase pathway and apoptosis are mediated by distinct regulatory subunits. Proc Natl Acad Sci U S A 99, 4221-6.

Simpson, P. (1990). Lateral inhibition and the development of the sensory bristles of the adult peripheral nervous system of Drosophila. Develop 109, 509-519.

Simpson, P., Woehl, R. and Usui, K. (1999). The development and evolution of bristle patterns in Diptera. Develop 126, 1349-1364.

Skeath, J. B. and Carroll, S. B. (1991). Regulation of achaete-scute gene expression and sensory organ pattern formation in the Drosophila wing. Genes \& Dev 5, 984-995.

Snell, V. and Nurse, P. (1994). Genetic analysis of cell morphogenesis in fission yeast-a role for casein kinase II in the establishment of polarized growth. EMBO J 13, 2066-2074.

Sommercorn, J., Mulligan, J. A., Lozeman, F. J. and Krebs, E. G. (1987). Activation of casein kinase II in response to insulin and to epidermal growth factor. Proc Natl Acad Sci U S A 84, 8834-8838.

Stifani, S., Blaumueller, C. M., Redhead, N. J., Hill, R. E. and Artavanis-Tsakonas, S. (1992). Human homologs of a Drosophila Enhancer of split gene product define a novel family of nuclear proteins. Nat Genet 2, 119-27.

Strack, S., Zaucha, J. A., Ebner, F. F., Colbran, R. J. and Wadzinski, B. E. (1998). Brain protein phosphatase $2 \mathrm{~A}$ : developmental regulation and distinct cellular and subcellular localization by B subunits. J Comp Neurol 392, 515-27.

Sun, X. and Artavanis-Tsakonas, S. (1997). Secreted forms of DELTA and SERRATE define antagonists of Notch signaling in Drosophila. Development 124, 3439-48.

Sun, Y., Jan, L. and Jan, Y. (1998). Transcriptional regulation of atonal during development of the Drosophila peripheral nervous system. Develop 125, 3731-3740.

Tamura, K., Taniguchi, Y., Minoguchi, S., Sakai, T., Tun, T., Furukawa, T. and Honjo, T. (1995). Physical interaction between a novel domain of the receptor Notch and the transcription factor RBP-J kappa/Su(H). Curr Biol 5, 1416-23.

Tata, F. and Hartley, D. A. (1995). Inhibition of cell fate in Drosophila by Enhancer of split genes. Mech Dev 51, 305-315.

Taylor, S. S. (1989). cAMP-dependent protein kinase: Model for an enzyme family. J Biol Chem 264, 8443-8446.

Thompson, B. J., Mathieu, J., Sung, H. H., Loeser, E., Rorth, P. and Cohen, S. M. (2005). Tumor suppressor properties of the ESCRT-II complex component Vps25 in Drosophila. DeV Cell 9, 711-20.

Tien, A. C., Rajan, A. and Bellen, H. J. (2009). A Notch updated. J Cell Biol 184, 621-629. 
Tietze, K., Oellers, N. and Knust, E. (1992). Enhancer of SplitD, a dominant mutation of Drosophila, and its use in the study of functional domains of a helix-loop-helix protein. Proc Natl Acad Sci U S A 89, 6152-6156.

Tomlinson, A. and Struhl, G. (2001). Delta/Notch and Boss/Sevenless signals act combinatorially to specify the Drosophila R7 photoreceptor. Mol Cell 7, 487-95.

Tournier-Lasserve, E., Iba-Zizen, M. T., Romero, N. and Bousser, M. G. (1991). Autosomal dominant syndrome with strokelike episodes and leukoencephalopathy. Stroke 22, 1297-302.

Trott, R. L., Kalive, M., Karandikar, U., Rummer, R., Bishop, C. P. and Bidwai, A. P. (2001a). Identification and characterization of proteins that interact with Drosophila melanogaster protein kinase CK2. Mol Cell Biochem 227, 91-98.

Trott, R. L., Kalive, M., Paroush, Z. and Bidwai, A. P. (2001b). Drosophila melanogaster casein kinase II interacts with and phosphorylates the basic-helix-loop-helix (bHLH) proteins m5, m7, and m8 derived from the Enhancer of split complex. J Biol Chem 276, 2159-2167.

Tsunematsu, R., Nakayama, K., Oike, Y., Nishiyama, M., Ishida, N., Hatakeyama, S., Bessho, Y., Kageyama, R., Suda, T. and Nakayama, K. I. (2004). Mouse Fbw7/Sel-10/Cdc4 is required for notch degradation during vascular development. J Biol Chem 279, 9417-23.

Uemura, T., Shiomi, K., Togashi, S. and Takeichi, M. (1993). Mutation of twins encoding a regulator of protein phosphatase $2 \mathrm{~A}$ leads to pattern duplication in Drosophila imaginal discs. Genes Dev 7, 429-40.

Vaccari, T. and Bilder, D. (2005). The Drosophila tumor suppressor vps25 prevents nonautonomous overproliferation by regulating notch trafficking. Dev Cell 9, 687-98.

Virshup, D. M. and Shenolikar, S. (2009). From promiscuity to precision: protein phosphatases get a makeover. Mol Cell 33, 537-45.

Wang, W. and Struhl, G. (2004). Drosophila Epsin mediates a select endocytic pathway that DSL ligands must enter to activate Notch. Development 131, 5367-80.

Wassarman, D. A., Solomon, N. M., Chang, H. C., Karim, F. D., Therrien, M. and Rubin, G. M. (1996). Protein phosphatase 2A positively and negatively regulates Ras1-mediated photoreceptor development in Drosophila. Genes \& Dev 10, 272-8.

Welshons, W. J. (1956). Dosage experiments with split mutants in the presence of an enhancer of split. Dros. Inf. Serv 30, 157-158.

White, N. and Jarman, A. (2000). Drosophila atonal controls photoreceptor R8-specific properties and modulates both receptor tyrosine kinase and Hedgehog signalling. Develop 127, 1681-1689.

Wilson, J. J. and Kovall, R. A. (2006). Crystal structure of the CSL-Notch-Mastermind ternary complex bound to DNA. Cell 124, 985-96.

Winkler, K. E., Swenson, K. I., Kornbluth, S. and Means, A. R. (2000). Requirement of the prolyl isomerase Pin1 for the replication checkpoint. Science 287, 1644-7.

Wolff, T. and Ready, D. (1991). The beginning of pattern formation in the Drosophila compound eye: the morphogenetic furrow and the second mitotic wave. Develop 113, 841850.

Wolpert, L. (1978). Pattern formation in biological development. Sci Am 239, 154-64. 
Xing, Y., Xu, Y., Chen, Y., Jeffrey, P. D., Chao, Y., Lin, Z., Li, Z., Strack, S., Stock, J. B. and Shi, Y. (2006). Structure of protein phosphatase 2A core enzyme bound to tumorinducing toxins. Cell 127, 341-53.

Xu, Y., Xing, Y., Chen, Y., Chao, Y., Lin, Z., Fan, E., Yu, J. W., Strack, S., Jeffrey, P. D. and Shi, Y. (2006). Structure of the protein phosphatase 2A holoenzyme. Cell 127, 1239-51.

Yu, I. J., Spector, D. L., Bae, Y. S. and Marshak, D. R. (1991). Immunocytochemical localization of casein kinase II during interphase and mitosis. J Cell Biol 114, 1217-32. 